\title{
RAMAN AND FTIR IMAGING OF DYNAMIC POLYMER SYSTEMS
}

\author{
by \\ JOHN PETER BOBIAK
}

\begin{abstract}
Submitted in partial fulfillment of the requirements for the degree of Doctor of Philosophy
\end{abstract}

\author{
Dissertation Advisor: Dr. Jack L. Koenig \\ Department of Macromolecular Science and Engineering \\ CASE WESTERN RESERVE UNIVERSITY
}

January, 2006 


\section{CASE WESTERN RESERVE UNIVERSITY \\ SCHOOL OF GRADUATE STUDIES}

We hereby approve the dissertation of

\section{John P. Bobiak}

candidate for the Ph.D. degree *.

(signed) $\frac{\text { Jack L. Koenig }}{\text { (chair of the committee) }}$

Alexander Jamieson

Hatsuo Ishida

M. Cather Simpson

(date) 22 November 2005

*We also certify that written approval has been obtained for any proprietary material contained therein. 
Copyright (C) 2005 by John Peter Bobiak

All rights reserved 


\section{Dedication}

This work is dedicated to my grandfather, Adolph. "It always takes longer to prepare for a job than it takes to do the job." 


\section{Table of Contents}

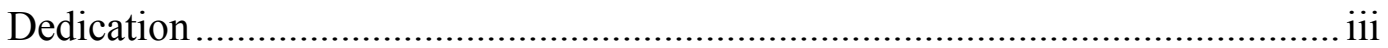

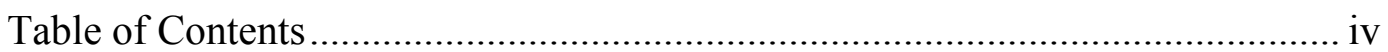

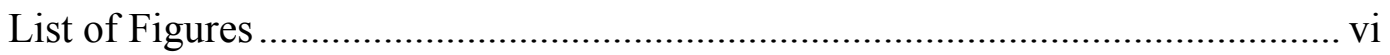

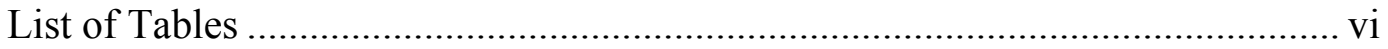

Acknowledgements ..................................................................................... xi

1 Raman and FTIR Imaging of Dynamic Polymer Systems............................... 1

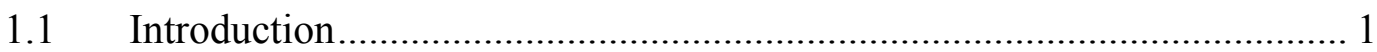

$1.2 \quad$ IR and Raman Imaging Background................................................. 2

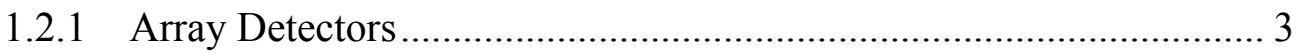

1.2.2 Applications of Focal Plane Arrays …………................................... 3

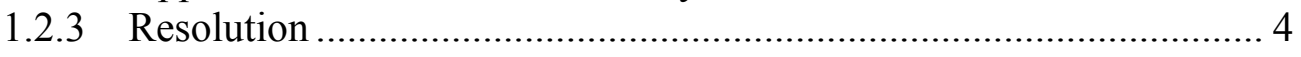

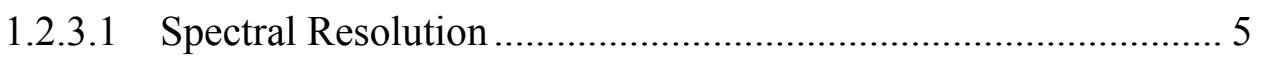

1.2.3.2 Spatial Resolution .................................................................. 7

1.2.3.3 Temporal Resolution.............................................................. 9

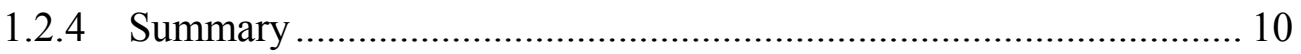

1.3 Infrared Imaging and Materials Applications .......................................... 10

1.3.1 IR Image Acquisition Schemes......................................................... 10

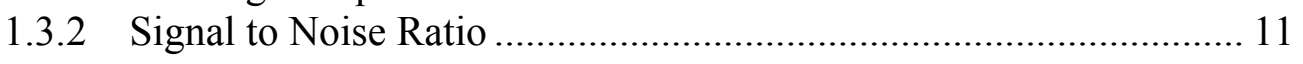

1.3.2.1 Signal Acquisition Schemes ..................................................... 12

1.3.2.2 Image Transformation Schemes ………………….................... 12

1.3.2.3 Post-FT refinement ............................................................... 13

1.3.3 Materials Applications .................................................................. 14

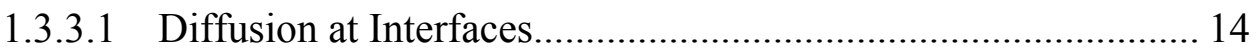

1.3.3.2 Release from Particles and Dispersions .................................... 16

1.3.3.3 Polymer Dissolution ............................................................... 18

1.3.3.4 Phase Transitions and Agglomeration ....................................... 20

$1.4 \quad$ Raman Imaging and Material Applications ............................................. 23

1.4.1 Instrument Features...................................................................... 23

1.4.2 Spatial broadening, confocality, and refraction ............................... 23

1.4.3 Image Acquisition Schemes............................................................... 24

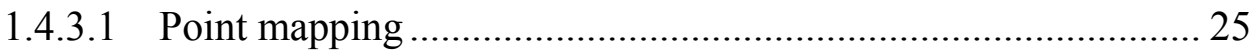

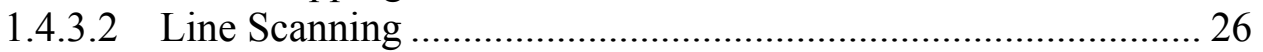

1.4.3.3 Global Illumination.............................................................. 26 
1.4.4 Materials Applications

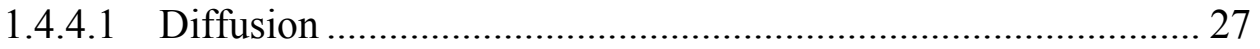

1.4.4.2 Processing Conditions........................................................... 29

1.4.4.3 Chemical modification of substrates....................................... 30

1.4.4.4 Phase/ Structural Transitions ................................................. 31

1.5 Other Raman and FTIR Imaging Applications ..................................... 34

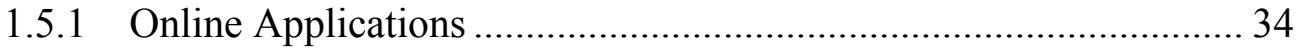

1.5.2 Parallel Detection Techniques ................................................. 34

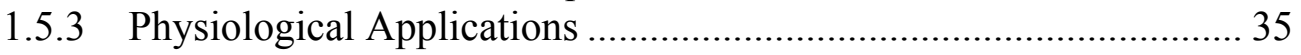

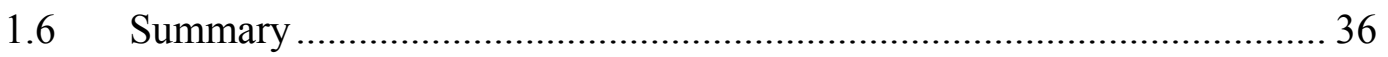

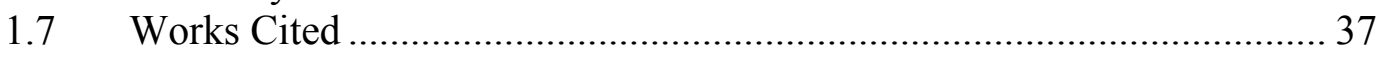

2 FTIR Imaging of Stereoregular Poly(methyl methacrylate) Dissolution ..... 52

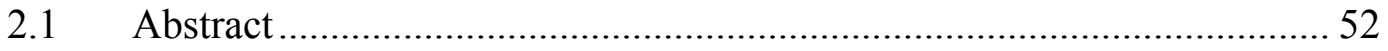

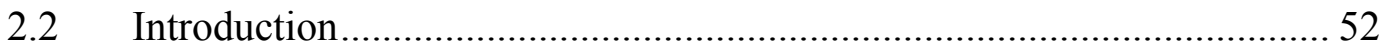

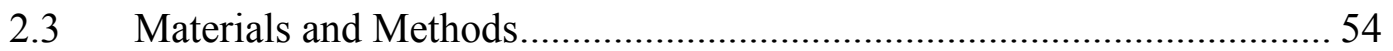

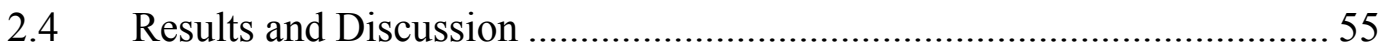

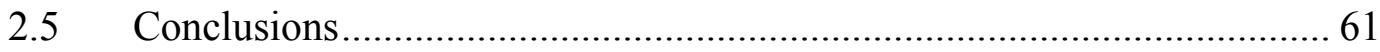

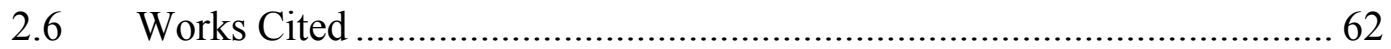

3 Regions of Interest in FTIR Imaging Applications: Diffusion of nicotine into Ethylene-co-Vinyl Acetate films ................................................................................ 74

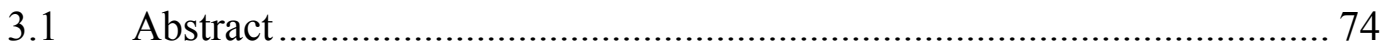

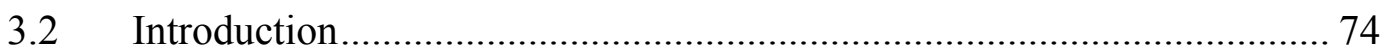

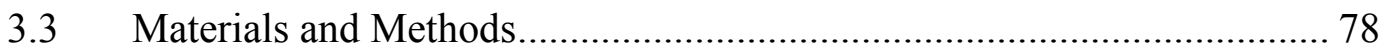

3.4 Results and Discussion .................................................................... 80

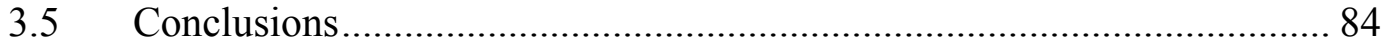

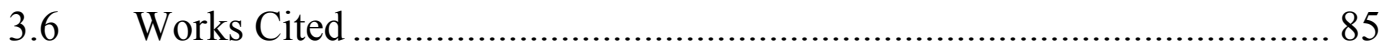

4 Structural Classification of Dispersed Single Walled Carbon Nanotubes by

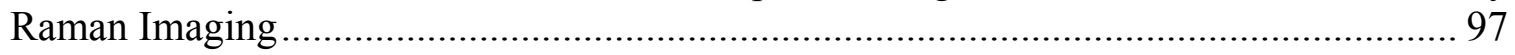

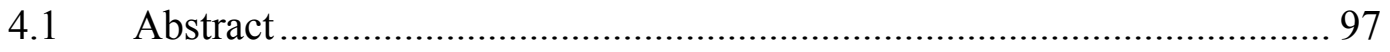

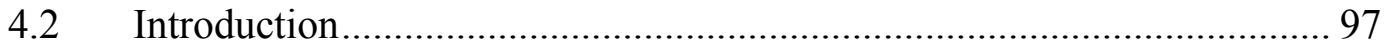

4.3 Materials and Methods....................................................................... 99

4.4 Results and Discussion ................................................................ 100

4.5 Conclusions ............................................................................... 104

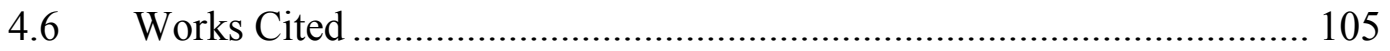




\section{List of Tables}

\section{CHAPTER 1}

Table 1.A. Origins of resolution dimensions for spectral imaging.

\section{CHAPTER 2}

Table 2.1. Characteristics of the solvents tested. Solubility parameter, molar volume and hydrogen bonding group from ref (45). Distance from PMMA calculated by: $\left[4\left(\delta_{\mathrm{d} 2}-\delta_{\mathrm{d} 1}\right)^{2}+\left(\delta_{\mathrm{p} 2}-\delta_{\mathrm{p} 1}\right)^{2}+\left(\delta_{\mathrm{h} 2}-\delta_{\mathrm{h} 1}\right)^{2}\right]^{1 / 2} \operatorname{ref}(46)$ 66 


\section{List of Figures}

\section{CHAPTER 1}

Figure 1.1. The mechanism for chemical contrast displayed for a PMMA/ 2ethoxyethanol interface.

Figure 1.2. Development of a hyperspectral cube by FTIR imaging and Raman line scanning. In FTIR imaging, steps of the spectrometer develops the wavelength ( $\lambda$ ) dimension. In Raman line scanning, stage rastering develops one spatial (y) dimension.

Figure 1.3. Schematic illustration of various array detector uses.

Figure 1.4. Schematic diagram of filtration-based infrared (a) and Raman (b, c) global imaging schemes.

Figure 1.5. Illustration of the $d_{\text {actual }}$ value for determination of the degradation factor.

Figure 1.6. Approximate duration of instrumental processes while acquiring Infrared (dashed lines) and Raman (solid lines) Images. Processes shared by some techniques are indicated by double solid lines.

Figure 1.7. Schematic diagrams for step-scanning acquisition schemes (a) and continuous-scanning acquisition schemes (b).

Figure 2.1. Dissolution of isotactic PMMA in benzene: a) before solvent addition; and after b) $2 \mathrm{~min}$, c) $6.6 \mathrm{~min}$, d) $11.25 \mathrm{~min}$, and e) $16 \mathrm{~min}$ of solvent exposure. 
iPMMA images plotted using the absorbance at $1755 \mathrm{~cm}^{-1}$; benzene images plotted using the absorbance at $1478 \mathrm{~cm}^{-1}$.

Figure 2.2. Eruption of aPMMA polymer fragments when exposed to benzene: a) before solvent addition; after b) 2 minutes; c) $35 \mathrm{~min}$; d) $46 \mathrm{~min}$; e) $51 \mathrm{~min}$; f) $57 \mathrm{~min}$ of solvent exposure.

Figure 2.3. Normalized absorbance profiles of iPMMA dissolving in benzene: a) before solvent addition; and after b) $2 \mathrm{~min}$, c) $6.6 \mathrm{~min}$, d) $11.25 \mathrm{~min}$, and e) $16 \mathrm{~min}$ of solvent exposure.

Figure 2.4. Images of iPMMA dissolving in 2-ethoxyethanol: a) before solvent addition; b) 2 minutes; c) $8 \mathrm{~min}$; d) $14 \mathrm{~min}$; e) $22 \mathrm{~min}$; f) $30 \mathrm{~min}$; g) $37 \mathrm{~min}$; h) $44 \mathrm{~min}$; i) $51 \mathrm{~min}$; j) $60 \mathrm{~min}$. Images generated by plotting the absorbance at $1750 \mathrm{~cm}^{-1}$ for iPMMA and $2857 \mathrm{~cm}^{-1}$ for 2-ethoxyethanol..

Figure 2.5. Normalized absorbance profiles for the iPMMA/ 2-ethoxyethanol system at: a) $2 \mathrm{~min}$, b) $25 \mathrm{~min}$, c) $44 \mathrm{~min}$, and d) $60 \mathrm{~min}$. Profiles shown for absorbances at $1750 \mathrm{~cm}^{-1}, 1705 \mathrm{~cm}^{-1}, 2857 \mathrm{~cm}^{-1}$.

Figure 2.6. Displacement of polymer front versus time for three tacticities of PMMA films exposed to acetic acid.

Figure 2.7. Displacement of the iPMMA front upon exposure to various solvents.

Figure 3.1. Mean integrated absorbance and deviation of a nicotine-specific band within one region of interest increases over time. Test statistic, $t_{0}$, is also noted for each 
time. The nicotine concentration within Column \#38 is concluded to have increased only after $t_{0}$ exceeds the reference statistic, $t_{0.05,30}=1.697$. The absorbance in Column \#38 increased after 17 minutes.

Figure 3.2. The characteristic nicotine absorbance near the diffusion front increases as diffusion proceeds over time. The process is represented by a) a statistical profile and b) a raw absorbance profile. The arrows in $2 \mathrm{a}$ and $2 \mathrm{~b}$ indicate the reported position of the nicotine diffusion front, based either on comparison the threshold value (2a) or subjectively based on the profile shape (2b).

Figure 3.3. Progress of the nicotine diffusion front over time estimated from using the test statistic approach (a) and the raw absorbance profile (b). Linear fits are used since the diffusion process is expected to be Fickian.

Figure 3.4. The average spectrum from a region of interest employing sizes, $n$, of: 1, 3, 7 , 11, and 19 pixels.

Figure 3.5. Mean centered spectra in the characteristic spectral region of nicotine. Plot indicates of the improved noise level as $\mathrm{n}$ increases.

Figure 3.6. Comparison of diffusion kinetics as estimated by determination limit (a) and the test statistic approach (b).

Figure 3.7. Nicotine permeation into EVA film from different solution compositions: no surfactant, non-ionic surfactant (Tween 40), anionic surfactant (SDS) 
Figure 3.8. Displacement of the nicotine diffusion front (micron) over time $\left(\min ^{1 / 2}\right)$ for two different surfactant identities, Tween 40 and Tween 80; either surfactant was present at $10 \mathrm{wt} \%$ in the solution. The results from two independent trials under the same conditions are shown to illustrate reproducibility.

Figure 4.1. Image of dispersed single walled carbon nanotubes based on $\mathrm{G}$ band intensity $\left(1500-1620 \mathrm{~cm}^{-1}\right)$.

Figure 4.2. Histograms for the integrated intensity values in the spectral region of the $G$ band (a) and an empty spectral region (b). The raw spectral data ( $\mathbf{a})$ was preprocessed by either median filtration $(\circ)$ or IFT $(\boldsymbol{\nabla})$.

Figure 4.3. Histogram of the $\mathrm{I}_{\mathrm{D}} / \mathrm{I}_{\mathrm{G}}$ value for upon median filtration of the raw spectral data, displayed for all pixels in the image ( $\boldsymbol{\square})$ and for only those pixels with Gband intensity greater than $13(\circ)$.

Figure 4.4. Histograms of the $\mathrm{I}_{\mathrm{D}} / \mathrm{I}_{\mathrm{G}}$ values of thresholded images without spectral preprocessing (a), with median filtration of the spectral data (b), and with IFT truncation of the spectral data (c).

Figure 4.5. Histograms of the $\mathrm{I}_{\mathrm{D}} / \mathrm{I}_{\mathrm{G}}$ values for images acquired by point-mapping (solid) and line scanning (open): unprocessed spectral data (a), median-filtered spectral data (b), and IFT truncated spectral data (c).

Figure 4.6. Scatter plots of $I_{G}$ versus the $I_{D} / I_{G}$ value for: pixels in the unprocessed image (a), the median filtered image (b), and the IFT truncated image (c). 


\section{Acknowledgements}

I offer my gratitude to Professor Koenig for taking just one more student, myself, under his wing. It has been an honor to work for him. A special thanks to Barbara and Barbara for supporting the group. I would also like to acknowledge several former members of the Koenig Group: Carrie, Regan, Beth, Guiyang, and Yuji.

My lasting thanks to Sarah for her constant love and support.

Finally, I would like to acknowledge the National Science Foundation Division of Materials Research, for financial support under contract DMR-0100428. 


\section{List of Abbreviations}

aPMMA - atactic poly (methyl methacrylate)

ATR - attenuated total reflectance

$\mathrm{CaF}_{2}$ - calcium fluoride

$\mathrm{CCD}$ - charge coupled detector

$\mathrm{CMC}$ - critical micelle concentration

D band - "disorder" induced band in the Raman spectrum of carbon materials, typically in the range of $1200-1360 \mathrm{~cm}^{-1}$

$\mathrm{D}_{2} \mathrm{O}$ - deuterium oxide

EPR - ethylene-propylene rubber

EVA - poly (ethylene-co-vinyl acetate)

FPA - Focal Plane Array

FTIR - Fourier transform infrared

G band - in-plane C-C stretching band in the Raman spectrum of carbon materials, typically in the range of $1500-1620 \mathrm{~cm}^{-1}$

HSP- Hansen solubility parameter

$I_{D}$ - intensity of the $D$ band

$I_{D} / I_{G}-$ ratio between $I_{D}$ and $I_{G}$

$I_{G}$ - intensity of the $G$ band

iPMMA - isotactic poly (methyl methacrylate)

iPP - isotactic poly (propylene)

IR - infrared

LC - liquid crystal 
MIBK - methyl isobutyl ketone

$\mathrm{M}_{\mathrm{w}}$ - weight average molecular weight

PAMS - poly (alpha-methyl styrene)

PE - poly (ethylene)

PEG - (poly (ethylene glycol)

PEO - poly (ethylene oxide)

PMMA - poly (methyl methacrylate)

PP - poly (propylene)

PVAc - poly (vinyl acetate)

PVC - poly (vinyl chloride)

PVP - poly (vinyl pyrrolidone)

ROI - region of interest

SDS- sodium dodecyl sulfate

SNR - signal to noise ratio

sPMMA - syndiotactic poly (methyl methacrylate)

SWCNT - single walled carbon nanotube

$\mathrm{T}_{\mathrm{g}}$ - glass transition temperature

$\lambda$ - wavelength 
Raman and Infrared Imaging of Dynamic Polymer Systems

Abstract

by

JOHN PETER BOBIAK

This work aims to expand the applications of Raman and infrared imaging in materials science and engineering. Recent developments in spectroscopic imaging technology have led to relatively fast image acquisition rates, enabling the in situ analysis of various engineering processes. A brief review of spectroscopic imaging principles and existing applications is provided as background before three novel applications are set forth.

First, the effectiveness of FTIR imaging for modeling polymer dissolution behavior was examined in a series of binary poly (methyl methacrylate) (PMMA) systems. The dissolution behavior was influenced by polymer conformation as well as the solvent characteristics. The results indicate that chemistry alone is a poor predictor of dissolution rate. Rather, the diffusion coefficients of both the polymer and solvent have a foremost impact on the dissolution process.

One major complication in modeling diffusion-related process by FTIR imaging is the precise determination of component locations in a series of images. This issue is addressed through the introduction of a new position-reporting technique based on hypothesis testing. A rudimentary drug release system, consisting of a poly (ethylene-covinyl acetate) film and a nicotine solution, was used to illustrate the importance of precisely reporting the nicotine diffusion front position. The new reporting method 
provided an inherent level of certainty to the position report. This method was applied to qualitatively assess the uptake of nicotine from solutions containing different solubilizing agents, which were capable of either promoting or inhibiting nicotine uptake.

Finally, Raman mapping and Raman line imaging were used to classify individual carbon nanotubes that were dispersed on a substrate. Individual nanotubes displayed a range of spectral characteristics, indicating that the bulk sample was a mixture of materials with different graphitic domain sizes. The results from images acquired by Raman mapping were identical to those acquired by line imaging. These results establish line imaging as a relatively fast, effective method to analyze heterogeneous nanotube samples. 


\section{Raman and FTIR Imaging of Dynamic Polymer Systems}

\subsection{Introduction}

The spatial arrangement of components in a materials system influences material properties - this is the central concept of material science. Accordingly, knowledge of both chemical and spatial characteristics is essential for solving problems in material science. Bulk spectroscopic techniques provide chemical and concentration information without microscopic spatial discrimination; similarly, many imaging techniques provide morphological information without chemical specificity. Spectroscopic imaging is the collection of spectroscopic information with defined resolution in the spatial and/or temporal domains. Among characterization tools, spectroscopic imaging has provided unique insights to the way materials respond on a microscopic scale to various stimuli: electrical, thermal, physical and chemical. This work aims to review the fundamental aspects of infrared and Raman imaging techniques and to extend the applications of those techniques.

Infrared and Raman spectroscopies are particularly powerful for characterizing macromolecular systems including blends, mixtures, and composites. Microscopic IR and Raman techniques have enabled the characterization of matrix materials and dispersed impurities, the observation of compositional gradients, and the quantification polymer orientation among other things ${ }^{1}$. The relatively recent development of multichannel detection devices has allowed images to be acquired in a fraction of the time required by earlier spectroscopic imaging methods. Thus, the base of applications 
for infrared and Raman imaging techniques has expanded tremendously. The most recent applications have involved real-time analysis of systems that change over time.

\subsection{IR and Raman Imaging Background}

Early infrared and Raman imaging devices consisted of spectroscopic light sources and detectors coupled to the optical paths of microscopes. Original image acquisition schemes involved collecting spectra at discrete positions on a point-by-point basis through automated or manual movement of a microscope stage. The chemical contrast mechanism for visualizing the distribution of different chemical species is illustrated in Figure 1.1. The infrared spectra of two materials, a polymer and a solvent, are displayed in Figure 1.1a. The intensity of a component-specific absorbance band can be plotted for each point where a spectrum was collected, producing a band-specific image. Figure $1.1 \mathrm{~b}$ shows that intensity values are recorded at several frequencies for each position in the sample, this form of three-dimensional matrix of intensity values is often called a data cube. Extraction of the intensity information at a component-specific frequency can lead to the formation of a component-specific image in which the intensity values are simply color-coded. Figure $1.1 \mathrm{~b}$ portrays a polymer-solvent interface with gradients of both components.

Point-mapping acquisition strategies produced long acquisition times since the sources and/or single-channel detection devices had to perform at each selected position of the sample. Current imaging strategies offer significantly shorter acquisition times, high spectral resolution, and spatial resolution close to the diffraction limit due to 
improvements in optical configurations, detector technology, and signal processing methods.

\subsubsection{Array Detectors}

Array detectors are an indispensable component of modern hyperspectral imaging methods. Focal plane array (FPA) detectors used for chemical imaging consist of a twodimensional grid of independently responding elements (pixels) arranged in the image plane of a microscope. The functions of FPAs in vibrational spectroscopic techniques have been reviewed elsewhere ${ }^{2}$. The signal for each pixel in the array can be independently read and stored at designated times in the acquisition scheme, providing a multichannel advantage which supersedes the need for point-by-point mapping schemes. The signals collected during image acquisition leads to the formation of a data cube: a three dimensional matrix of intensity values.

\subsubsection{Applications of Focal Plane Arrays}

FPA detectors have a range of capabilities in vibrational spectroscopy. In terms of data cube formation, both directional spatial components of the FPA (either the x-component or the y-component) can impart one dimension of resolution while another system component imparts a third dimension to the data cube. As implied before, exactly which dimensions are imparted by the FPA - spatial $(x)$, spatial $(y)$, wavelength $(\lambda)$, or temporal $(t)$ - depend on the instrument configuration and the acquisition scheme. Figure 1.2a illustrates the development of a data cube in FTIR imaging, in which the FPA imparts two spatial dimensions while movement of the interferometer imparts the frequency dimension. Figure $1.2 \mathrm{~b}$ illustrates the development of a data cube in Raman line 
imaging, in which the FPA imparts the wavelength dimension and one spatial dimension while rastering of the sample imparts the second spatial dimension.

In global acquisition schemes, like FTIR imaging, the microscope's optical train assures that spatial characteristics within the sample plane are retained upon projection to the FPA. The intensity of light detected at some pixel $\left(\mathrm{x}_{\mathrm{FPA}}, \mathrm{y}_{\mathrm{FPA}}\right)$ in the FPA directly corresponds to the absorption behavior of the material the sample plane ( $\left.\mathrm{x}_{\text {sample }}, \mathrm{y}_{\text {sample }}\right)$. The FPA operates differently in a line-scanning Raman acquisition scheme: scattered frequencies from adjacent sample positions are dispersed across one component of the array detector, while the other detector component provides one dimension of spatial resolution. Figure 1.3 illustrates a few array detector functions that have been employed, including those for FTIR imaging (Figure 1.3a) and Raman line-scanning (Figure 1.3b). Through a coordinated signal acquisition scheme, one component of the array could impart spatial resolution while the other component imparts temporal resolution, as in Figure 1.3c. One component of the FPA could impart spectral resolution while the other component imparts temporal resolution, as in Figure 1.3d. Arrays also offer the opportunity to improve SNR by binning adjacent pixels as in Raman point mapping.

\subsubsection{Resolution}

Applications of particular spectral imaging techniques are limited by a number of factors: the identity and physical state of the sample; the stability of the sample during image acquisition; and the size of spatial features that need to be observed. In this review the term spatial resolution refers to distance (micron) between features in the sample plane; spectral resolution refers to frequency ranges in the electromagnetic spectrum and the SNR in those ranges; temporal resolution refers to the time required to acquire a raw data 
cube. The ability to analyze a process in situ requires that: 1) multiple images can be acquired throughout the process, and 2) that the physical or chemical changes can be detected in the spatial or spectral dimensions.

\subsubsection{Spectral Resolution}

Spectral resolution refers to the ability to discern the intensities of different frequencies of the electromagnetic spectrum. Only the visible and infrared regions of the spectrum are of interest for vibrational spectroscopy. Resolution in the spectral dimension of a data cube provides chemical contrast in any spectral imaging technique. The fidelity of a band-specific image relies on resolution in the spectral domain. There are three general approaches for providing spectral resolution to any IR and Raman measurement: multiplex methods, dispersive methods, and filtration methods. In terms of imaging, the spectral resolving method essentially dictates the way that an FPA is utilized. This section focuses on the first aspect of spectral resolution: strategies to discern different frequencies of light. Strategies to improve spectral signal to noise ratio (including signal averaging, smoothing, and principle component analysis) in imaging applications will be mentioned in the FTIR imaging or Raman mapping sections, respectively.

\subsection{FTIR imaging and IR imaging}

FTIR imaging employs an interferometer to modulate a broadband IR source which

illuminates the sample ${ }^{3}$. The light passing through the sample is detected by all pixels in the FPA at discrete interferometer positions, and image acquisition involves the formation of an interferogram at each pixel. A Fourier transform can be applied to each 
interferogram to generate a spectrum for each pixel. Scanning of the interferometer necessarily imparts spectral resolution. Obtaining higher levels of frequency discrimination requires signal acquisitions at larger mirror retardations.

As an alternative to spectrometer movement, the sample may be illuminated by only a narrow infrared frequency range - this can be achieved by filtration of the broadband infrared source. Since the light illuminating the sample is already spectrally resolved, the acquired signal doe not require transformation to generate a frequencyspecific image. In filtration approaches, development of a spectral dimension of the data cube requires change to the filter's bandpass. The attainable spectral resolution of such filtration methods is limited by the bandpass of the filter.

Regardless of which method (multiplex or filtration) is used to provide spectral resolution to an infrared image, the vertical and horizontal components of the FPA impart two spatial dimensions to the data cube.

\subsection{Raman imaging}

Instrumental designs based on dispersive elements transmit spectral information across one component of the FPA, whereas those designs based on filtering elements project two dimensions of spatial information across the two components of the FPA. All Raman measurements require attenuation of Rayleigh-scattered light and efficient detection of the Stokes or anti-Stokes scattered frequencies. It is also important to note that current multiplex (FT) Raman methods do not retain spatial encoding of the scattered frequencies, limiting the utility of array detectors for multiplex Raman imaging. 
Image acquisition with instruments employing a dispersive element typically requires sample rastering to develop a data cube. The need for rastering can be eliminated by using a wavelength filtration-based global acquisition scheme. The FPA in filtration-based designs imparts two dimensions of spatial resolution in the same way that FPAs are employed in IR imaging: a large sample area is illuminated while a bandpass filter is placed between the sample and the FPA. It is important to realize that the inelastically scattered light from a certain molecular vibration is merely shifted from the illuminating frequency, thus there are two possibilities for filtering a globally illuminated Raman image: 1) using a constant illuminating frequency while tuning the filter bandpass, 2) tuning the frequency of the illuminating source while keeping the filter bandpass constant. Filtration-based imaging schemes, for both IR and Raman, are illustrated in Figure 1.4. As in IR imaging, the attainable spectral resolution of these methods is limited by the bandpass of the filter and development of a spectral dimension to the data cube requires modulation of either the bandpass or laser frequency. Global Raman imaging methods suffer from two complications: 1) diffusion of a laser beam across a relatively large area very yields low power densities, ultimately causing a very low degree of Raman scattering, 2) fluorescence from any species in the illuminated area can overwhelm the Raman signal, resulting in poor spectral discrimination.

\subsubsection{Spatial Resolution}

Microscope objective lenses are applied in chemical imaging to illuminate a defined region of the sample. In global illumination schemes the field of view is fixed and movement of the sample is not required. Spectral information for all of the illuminated 
volume elements in the sample plane is collected in parallel. In point illumination schemes, only a single volume element are illuminated at once, so sample rastering is required to generate an image: spectral information from volume elements is collected in sequence. The diffraction limit (discussed below) provides a practical upper limit to the number of volume elements that comprise the field of view.

The use of powerful objectives or smaller pixels in the FPA does not provide infinitely fine discrimination of spatial features. Diffraction ultimately limits the minimum distance between two discernable objects. For light of wavelength $\lambda$, the Rayleigh criterion requires that objects be separated by a distance, $r$, to be resolvable:

$$
r=\frac{1.22 \lambda}{2(n \sin \Theta)}
$$

Where $(n \sin \theta)$ is the numerical aperture (NA) of the microscopic objective lens. In typical infrared transmission and Raman backscattering techniques, $(\sin \theta)$ is equal to unity. Thus, the lateral resolution can be improved either by using lower incident wavelengths or by contacting the sample with media of high refractive index, such as an ATR element in FTIR imaging or an immersion oil in Raman imaging. Most FTIR transmission images use an objective with an NA close to 0.9 , resulting in theoretical spatial resolutions of approximately 1.7 microns at $4000 \mathrm{~cm}^{-1}$ and 13 microns at $500 \mathrm{~cm}^{-1}$. Due to engineering limitations, instruments seldom offer information at the theoretical diffraction limit. The spatial resolution provided by an instrument can be evaluated by a performance characteristic called the degradation factor, which is simply the ratio 
between the experimentally determined lateral resolution $\left(d_{\text {actual }}\right)$ and the diffractionlimited theoretical lateral resolution $\left(d_{\text {theoretical }}\right)^{4}$ :

$$
\frac{d_{\text {actual }}}{d_{\text {theoretical }}}=(\text { Degradation Factor })
$$

The $\mathrm{d}_{\text {actual }}$ value is defined as the observed width of a sharp interface, which is often taken as the lateral distance between points of $5 \%$ and $95 \%$ of a spectral band's intensity, see Figure 1.5. In FTIR imaging, the degradation factor of a transmission image is approximately 6.0, while that for a single bounce ATR image is approximately $1.3^{4}$. Even that level of degradation for the ATR resolution can be improved by almost $60 \%$ through the use of ATR elements which have higher refractive indices ${ }^{5}$.

\subsubsection{Temporal Resolution}

Most efforts in spectroscopic methods development aim to attain acceptable spectral resolution at high acquisition rates. In the acquisition of a data cube it often happens that increased resolution in one dimension (spatial, spectral, or temporal) comes at the expense of another. For instance, higher spectral resolution generally requires longer acquisition times, i.e. lower temporal resolution. Hyperspectral imaging often requires tradeoffs be made in order to attain acceptable resolution in all dimensions. Figure 1.6 depicts the approximate time scales for a variety of processes in infrared and Raman imaging including: frame rate (integration time), rastering period, and the acquisition time for a complete data cube. 
To illustrate these tradeoffs, consider the acquisition of a series of data cubes, first by Raman point-mapping then by FTIR imaging. In Raman mapping, the data cube is built on a point-by-point basis. A finite integration time of the detector (on the order of seconds) imparts good spectral resolution and a period of rastering provides spatial resolution. The image acquisition time could be decreased by imaging few spots on the sample (providing lower spatial resolution) or by decreasing the integration time spent at each spot (resulting in lower spectral SNR). In contrast to point-mapping, spatial resolution in FTIR imaging is fixed but modulation of the IR source is needed to impart spectral resolution. The acquisition time could be decreased by faster light source modulation (employing a continuously scanning an interferometer for instance) which could degrade the spectral resolution. Some computational approaches for improving image quality without sacrificing times will be discussed later.

\subsubsection{Summary}

To this point, Raman and infrared imaging strategies have been discussed in the context of those features they have in common. Raman and infrared spectroscopies each have their own nuances. The applications of Raman and infrared imaging to polymer systems are discussed separately below; each discussion is based upon specific phenomena in materials science for which either Raman or infrared imaging technique has been applied.

\subsection{Infrared Imaging and Materials Applications}

\subsubsection{IR Image Acquisition Schemes}

The source, sample, and detection components of FTIR imaging instrumentation function identically to any FTIR spectrometer: radiation from a broadband source is modulated 
over time; specific frequencies of the radiation are absorbed by moieties in the sample; the transmitted light is detected and appropriately processed. The interferometer may scan in either a continuous or step-scanning motion. The distinguishing characteristics of an infrared imaging apparatus are the FPA detector and Cassegrain optical path. Array detectors offer a multichannel advantage which itself provides many opportunities in the area of post-acquisition data handling. Improvements in FTIR image acquisition schemes ${ }^{6}$ and hardware performance ${ }^{7}$ have provided relatively fast acquisition of reliable data cubes. Currently, FTIR imaging with a continuously scanning spectrometer is state-of-the-art. Continuous scanning enables data cubes to be developed approximately 6 times faster than the early step-scanning FTIR imaging approach ${ }^{8}$.

A number of other imaging approaches have addressed the demands for even faster acquisition or higher SNR. However, as mentioned previously, high resolution in one domain often decreases resolution in another. Tunable filters enable the acquisition of global images in a matter of seconds, albeit at the expense of spectral resolution ${ }^{9}$. Use of synchrotron infrared radiation can considerably improve SNR in micro-FTIR techniques ${ }^{10}$ but those sources cannot be effectively integrated with an FPA ${ }^{11}$.

\subsubsection{Signal to Noise Ratio}

Information in the spectral dimension has a significant impact on image fidelity. The sources and impact of spectral noise have been evaluated in the context of FTIR imaging

12. The aim of this section is to describe how spectral quality can be improved at each step of the imaging process. 


\subsubsection{Signal Acquisition Schemes}

To impart a spectral dimension to the FTIR imaging data cube, signal must be integrated from each pixel at each interferometer position. SNR can be improved by reading each pixel response several times per spectrometer step and coadding all of those values - this process is called frame coaddition ${ }^{13}$. Frame coaddition can be applied to either step scanning or continuous scanning modes of the spectrometer, see Figure 1.7. For small numbers of frames $(\mathrm{N})$, less than 12 , SNR improves by a factor but $\mathrm{N}^{0.5}$, but the performance of the $\mathrm{A} / \mathrm{D}$ converter diminishes the improvement for larger numbers of frame coadds. Raw intensity data can be treated prior to coaddition by intensity filtration - removing those frames with statistically outlying intensities ${ }^{14}$; this process, called median filtered time averaging, effectively removes the impact of random, high intensity frame integrations.

\subsubsection{Image Transformation Schemes}

During image acquisition, an interferogram is developed for each pixel of the data cube. Those interferograms can be handled like any independent interferogram before applying a Fourier transform routine. Several processes (apodization, truncation, etc.) could be applied to the interferograms to synthetically eliminate high frequency noise.

Interferogram intensities can also be normalized for each pixel as to employ the full range of the analog-to-digital converter (ADC) ${ }^{15}$ - this process, called gain ranging, has been shown to improve spectral SNR by $45 \%$. 


\subsubsection{Post-FT refinement}

After the pixel responses are transformed into spectral information, referencing procedures can be applied to remove background artifacts. Another coaddition process, called pseudo coaddition, can remove noise or background changes that occur on the time scale of image acquisition (on the order of minutes). Pseudo coaddition involves the referencing of a single sample image to several background images collected before and after the sample image ${ }^{16}$. The SNR improvement of this coaddition scheme does follow a square root dependence with the number of coadded images.

Several types of transformations in the spectral domain, including principle component analysis (PCA), minimum noise transformation (MNF), and Fast Fourier Transformation (FFT) can improve the image quality via reduction of spectral artifacts ${ }^{17}$ 18. Other intensity filtering and averaging operations in the spatial domain can improve the ability to visualize morphological features. Geometry of the sample in the image plane may allow the coaddition of similar pixels (in terms of chemical signatures), via sample coaddition ${ }^{16}$. In this routine, the entire spectrum (or intensity of a particular band) is averaged across a selected subset of pixels, a region of interest. This routine is particularly useful for the reduction of data from two spatial dimensions to one. The concept of sample coaddition has been extended to consider band intensity variances within a spatial region, enabling the detection of trace concentration changes over time by hypothesis testing ${ }^{19}$. 


\subsubsection{Materials Applications}

A number of important dynamic processes occur on the microscopic level in the field of polymer science and engineering: crystallization, immiscible phase agglomeration, diffusion, dissolution, and release. The capacity to capture hyperspectral images quickly makes FTIR imaging a powerful tool for analyzing such processes in situ. To date FTIR imaging has been used to monitor penetrant diffusion, drug release from polymer matrices, polymer dissolution, and phase separation of polymer blends. FTIR imaging holds a significant throughput advantage over point-mapping schemes, thus it has been applied for several ex situ morphological studies for which point-mapping could have been applied. The following sections summarize the application of FTIR imaging to solve different phenomenological problems involving macromolecules.

\subsubsection{Diffusion at Interfaces}

Macromolecules are often used as barrier materials. While the diffusion of small molecules into a polymeric material does rely on extrinsic system characteristics, it can also be influenced by chemical characteristics and composition of the polymer. Thus, it is important to understand the physiochemical relationships that influence diffusion at an interface. FTIR imaging is capable of selectively probing chemical and physical phenomena at a variety of interfaces: polymer-liquid, polymer-gas, and polymer-polymer. Among analytical methods used to monitor diffusion, not only is the mechanism of chemical contrast unique, it can also be applied in-situ.

Applications of liquid crystalline materials often employ the large property changes around a thermal transition temperature, the diffusion coefficient of a liquid 
crystalline material is one such material property. FTIR imaging has been used to investigate the diffusion of liquid crystalline material (4-n-pentyl-49-cyanobiphenyl) into poly (butyl methacrylate) around the nematic/ isotropic transition temperature 2021 . Unfortunately, the LC diffusion kinetics appeared independent of temperature due to the proximity of the nematic/isotropic transition to the $T_{g}$ of the polymer. This work was, however, the first to propose that concentration profiles extracted from FTIR imaging can supplement classic mass uptake investigations of diffusion.

Transdermal drug delivery involves the uptake of an active drug from a fluid reservoir, which subsequently permeates through a polymeric barrier and absorbs into the body. Composition of the fluid reservoir can influence the diffusion kinetics. A basic model for this drug delivery process focused on the uptake of nicotine from water/ ethanol/ nicotine mixtures into a polyethylene-co-vinyl acetate "barrier" ${ }^{22}$. Films exposed to water-nicotine mixtures (without ethanol) demonstrated the lowest rate of nicotine uptake. However, faster nicotine uptake corresponded with swelling of the polymer film induced by ethanol. Spectra extracted from the polymer-liquid interface confirmed that ethanol induced swelling of the polymer. Spectroscopic evidence also suggested that nicotine complexation with ethanol accounted for lower uptake rates in ethanol-nicotine mixtures (without water). Thus, the chemical activity of the reservoir components was unambiguously identified.

The physical state of drug formulations may be altered by the uptake of atmospheric moisture. Such a transition could ultimately influence release characteristics of a drug formulation. Moisture uptake by macroscopically heterogeneous PEO/ griseofluvin dispersions has been studied using an FTIR-ATR imaging apparatus ${ }^{23}$. 
Spectral evidence demonstrated preferential uptake of water by the polymer phase over that by the dispersed drug phase. The spatial characteristics of the components, another important parameter for drug release kinetics, did not change upon moisture uptake.

An application of FTIR imaging to study gaseous diffusion involves rubber oxidation ${ }^{24} 25$. A low degree of oxidative crosslinking near the rubber surface is desirable since it restricts subsequent oxygen diffusion without significantly deteriorating the rubber's mechanical properties. The extent of oxidation in the presence of different levels of stabilizer was monitored by tracing the $\mathrm{C}=\mathrm{C}$ bond absorbance over time - that absorbance decreased upon oxidation. The results showed that oxidation was merely delayed through the addition of high stabilizer concentrations, and those stabilizers prevented the formation of an impermeable (crosslinked) skin layer.

\subsubsection{Release from Particles and Dispersions}

The works mentioned above investigated the uptake of a mobile penetrant by a stationary polymeric material. To date, few published studies have employed the opposite design strategy: release of a mobile component from a matrix material. FTIR imaging offers a novel description of interfacial phenomena in designed release systems. An FTIR-ATR imaging apparatus offers the potential for convection and on-line UV-VIS measurements

during the study ${ }^{26}$ - two very important considerations for industrial application of the technique.

The level of a drug loading can impact the release characteristics of the therapeutic formulation. FTIR imaging has enabled in situ analysis of both the morphological attributes and the release profiles of testosterone from a PEO matrix ${ }^{8}$. 
The level of drug loading impacted the swelling behavior of the PEO by acting as a nucleating agent: smaller PEO spherulites (generated at lower loadings) swelled faster than larger spherulites. The amount of testosterone released into the aqueous media followed the PEO swelling profile only at lower drug loadings (below $20 \mathrm{wt} \%$ ). Release from formulations with higher loadings was not controlled by swelling of the matrix material. These findings provided mechanistic details that classic analytical methods would have overlooked.

As implied previously, the physical state of drug molecules often influences a drug's solubility and activity. Additives are frequently added to formulations to control the physical state of a molecule. For instance, formation of crystalline ibuprofen, which has a low solubility in aqueous media, can be prevented by the addition of cyclodextrin to the drug formulation. Ibuprofen release from an PEO matrix has been studied in situ by FTIR imaging ${ }^{27}$. Ibuprofen molecules were apparently released upon erosion of a PEG matrix but the drug molecules crystallized in the aqueous dissolution medium. In a real drug delivery system crystallization would have compromised the bioavailability of the drug. Crystallization was prevented by including cyclodextrin in a subsequent sample formulation. The cyclodextrin effectively increased the aqueous solubility of the ibuprofen molecules. FTIR imaging of this system allowed the simultaneous observations of multiple phenomena: dissolution of the matrix material, release of the ibuprofen, and formation (or lack there of) of the ibuprofen crystals. 


\subsubsection{Polymer Dissolution}

A number of important technologies, including drug release and photolithography, employ polymer dissolution either during fabrication stages or to impart specific functions to a device ${ }^{28}$. Accordingly, the characteristics of polymer dissolution influence the design of a process or a product. Just as in diffusion studies, the ability to observe both chemistry and kinematics of the polymer-solvent interface makes FTIR imaging a powerful tool for studying the dissolution process. The impacts of several system characteristics have been studied by FTIR imaging.

\subsection{Solvent characteristics}

The use of solvent combinations is one method to control the dissolution rate at a polymer-solvent interface. Binary mixtures of two good solvents ${ }^{29}$, one good solvent and one poor solvent ${ }^{30}$, and two poor solvents ${ }^{31}$ have been investigated. In the case of two good solvents at an interface with unentangled PAMS, simple mixing laws based on the composition of the solvent system were able to account for changes in the dissolution rate $^{29}$. Interestingly, low concentrations of a non-solvent in a binary solvent mixture increased the rate of dissolution of an unentangled PAMS sample as compared to a good solvent alone ${ }^{30}$. FTIR image analysis confirmed that this seemingly peculiar result was due to the formation of stronger concentration gradients in the systems containing small amounts of non-solvent.

Cosolvency is a dissolution phenomena in which a material dissolves in a mixture of two solvents, neither of which are capable of dissolving the material independently. 
The mechanism for cosolvency was investigated by FTIR imaging using a PMMA sample exposed to carbon tetrachloride/ methanol mixtures ${ }^{31}$. The spatially resolved spectra showed that methanol acted to hydrogen bond with the polymer while carbon teterachloride induced swelling of the polymer. The polymer did not exhibit preferential uptake of one solvent, rather the penetration of one solvent required the presence of the other. Only upon penetration of both solvents into the polymer did the PMMA chains relax and dissolve into the solvent reservoir.

\subsection{Polymer characteristics}

Chain entanglements develop as polymer molecular weight increases. Despite identical chemistry, an entangled system may undergo a considerably different dissolution process than an unentangled system. The dissolution of low molecular weight PAMS by MIBK ${ }^{29}$ was contrasted to that of high molecular weight PAMS ${ }^{32}$. While the interface of the low molecular weight material receded uniformly, the high molecular weight material demonstrated a fragmentation/ roughening process. The fragmentation behavior was caused by solvent uptake without rapid chain disentanglement; this produced an increased osmotic pressure which was relieved through fragmentation.

Stereoregularity of monomer units along a polymer backbone often influences the physical properties of a polymer. Despite identical chemistry and molecular weight, polymeric steroisomers may display considerably different behaviors upon exposure to a solvent. Three stereoisomers of PMMA of similar molecular weight have been exposed a variety of solvents in an FTIR imaging set-up ${ }^{33}$. Higher rates of dissolution were always observed for isotactic PMMA, owing to that material's comparatively bulky 
conformation. Even upon exposure to an unfavorable solvent, the isotactic PMMA chains were able to relax and dissolve while the atactic and syndiotactic stereoisomers demonstrated fragmentation in the same solvents. This evidence supported importance of chain configuration in empirical models for polymer dissolution rates.

\subsubsection{Phase Transitions and Agglomeration}

Certain stimuli can induce microscopic phase transitions or phase separations in polymer systems. Temperature, for instance, can control the growth of agglomerated domains, the diffusion of liquid crystalline material, or the crystallization of system components. FTIR imaging is effective for studying these types of systems as long as the phases have sufficient size and resolvable spectral characteristics. The primary advantage of fast chemical imaging techniques over microspectroscopy is the ability to study relatively rapid transitions in situ. Despite this advantage, few works have investigated in situ phase transitions using FTIR imaging. Samples are most often removed from a preparatory environment and the images are acquired under conditions where the sample is at equilibrium.

\subsection{In situ Investigations}

One study that indirectly employs the temporal advantage of FTIR imaging involved the agglomeration of diallyl phthalate agglomerates within a matrix of polybutadiene ${ }^{34}$. This model process could have not been sufficiently studied by FTIR mapping techniques since the phase transition occurred in randomly dispersed regions of the sample over the same duration that point-mapping would have required. The number of DAP 
agglomerates in the field of view decreased while the size of a few DAP agglomerates increased upon aging for 24 hours at room temperature. Agglomeration occurred because the polymer was above its glass transition temperature and the dispersed phase was not miscible with the matrix. As opposed to a mapping technique, FTIR imaging was beneficial for this study to assure minimal changes in the agglomerated state during image acquisition.

Thermally-induced liquid crystalline phase transitions have also been investigated in situ by FTIR imaging ${ }^{35}$. The orientation of a nitrile functional group within dispersed domains of E7 liquid crystal changed (with respect to the incident IR beam) upon transition from isotropic to nematic phases -this phase transition induced an observable change in the infrared image. Analytically, contrast of the phase-specific image was reduced by the concentrations of material dispersed in the polymer matrix that did not undergo the transition.

\subsection{Ex situ Investigations}

Many applications of FTIR imaging simply enable higher throughput than infrared pointmapping schemes. In most of the ex situ cases described here, similar conclusions could have been drawn using either global or point-mapping acquisition schemes.

Temperature changes can induce preferential crystallization of a polymer blend component and subsequent phase segregation. Segregation of PVAc dispersed in a PEO matrix was imaged by FTIR imaging ${ }^{36}$. Most of the amorphous PVAc material was forced into interspherulitic regions upon annealing the system near the PEO crystallization temperature. However, there was also an indication of higher 
concentrations near the center of the spherulite, suggesting that PVAc acted as a nucleating agent for the PEO.

The time scale on which temperature modifies morphological features depends on the nature of the materials at hand. FTIR imaging has been used to monitor thermally induced reactions, both at interfaces ${ }^{37} 38$ and within a dispersed phase ${ }^{39}$. In such systems, high temperatures increase both the diffusivity of reagents and the reaction kinetics of the curing reaction - thus the compositional gradients show complex temperature dependencies. Changes in temperature can cause the interfacial reaction kinetics to transition from diffusion-controlled (low temperature) to reaction controlled (high temperature).

Solvent induced phase transitions are generally investigated using bulk analytical methods (macro-FTIR, DSC, x-ray) which do not specifically probe surface characteristics. The mechanism for such transitions, however, necessarily involves diffusion of some chemical species at an interface. Thus, FTIR imaging is an ideal method for studying such transitions at an interface. The solvent induced crystallization of PET upon exposure to supercritical carbon dioxide $\left(\mathrm{SCCO}_{2}\right)$ is one exemplary system 40. Bulk methods have shown an increase of the trans isomer content (indicative of crystallization) upon exposure to a solvent, but spectral imaging of sample cross-sections confirmed that the isomerization occurred preferentially at the surface. FTIR imaging has also been used to study morphological development of hydroxyapatite from a Bioglass composite upon solvent exposure ${ }^{41}$ and solvent-induced phase separation of polymer blends ${ }^{42}$. 
Liquid crystalline domains within a polymer matrix can align upon exposure to an electric field, generating changes of refractive index of the LC domain. This morphological transition was been used to characterize the effects of refractive index on

FTIR image quality ${ }^{43}$. Spectral baseline artifacts often occur at material interfaces due to RI mismatches. The extent of the baseline shift was significantly reduced upon application of the electric field indicating that the refractive index of the LC approached that of the matrix material.

\subsection{Raman Imaging and Material Applications}

\subsubsection{Instrument Features}

Three instrumental features have prompted the recent resurgence of analytical Raman spectroscopy, including fast Raman imaging. First, microscopic focus of the incident laser beam generates high power densities in the focal plane, which increases the scattering intensities relative to an unfocussed laser source. Microscopic focus of the laser beam also enables spectral acquisition from defined sample areas. Second, the use of dielectric and holographic filters allows extremely efficient attenuation of the laser line without significant loss of inelastically scattered lines. Modern configurations enable the collection of several times more of the inelastically scattered light than designs with multiple dispersive elements. Finally, focal plane array detectors provide a multichannel advantage which speeds up the data acquisition process.

\subsubsection{Spatial broadening, confocality, and refraction}

Photon scattering can occur from any material illuminated by a light source. In Raman imaging applications, scattering from material outside of the focal plane can produce 
unexpected spectral artifacts, often degrading the spatial resolution of an image. In the xy sample plane, broadening of a sharp interface may be corrected by introduction of a characteristic instrumental broadening function ${ }^{44}$. Scattering from material along the $\mathrm{z}$ axis, however, cannot be categorically corrected by such a function.

Confocal apertures effectively filter light that originates from outside of a focused laser spot's volume. Optical confocal holes enable depth scanning of transparent samples by fluorescent and Raman imaging spectroscopies. The z-positioning of the laser spot in the sample can be controlled by simple adjustment of the microscope stage. Refraction at the sample-air interface, however, changes the volume of the illuminated laser $\operatorname{spot}^{4546}$. Refraction causes depth of the illuminated field to increase as the laser spot is focused deeper into the sample; even the use of confocal holes cannot correct for refraction effects in Raman imaging. Consequently, refraction dictates the fidelity of confocal Raman depth profiles. Two simple instrumental modifications can overcome the effect of refraction: use of cover-slip corrected objective lenses ${ }^{47}$ and use of immersion oil objective lenses ${ }^{46} 40$.

\subsubsection{Image Acquisition Schemes}

As mentioned previously, the time required to acquire a Raman image by any scheme (global, line scanning, or point mapping) directly depends on the duration of detector integration, or frame rates. Accordingly, species that exhibit intense scattering would require shorter frame rates. The radiant scattering flux, $\mathrm{I}_{\text {scattered }}\left(\mathrm{J} \mathrm{sr}^{-1}\right)$, relates to the

number of irradiated species, $\mathrm{N}$, the differential scattering cross section, $\frac{d \sigma}{d \Omega}\left(\mathrm{cm}^{2} \mathrm{sr}^{-1}\right)$,

and the illuminating exposure, $\mathrm{I}_{\text {excitation }}\left(\mathrm{J} \mathrm{cm}^{-2}\right)$ by equation: 


$$
I_{\text {scattered }} \approx N \bullet I_{\text {excitation }} \bullet \frac{d \sigma}{d \Omega}
$$

At a constant illuminating power, it can be seen that the size of the illuminated area (laser spot) influences the flux of Raman scattering. Point mapping schemes, which generally illuminate $10^{0}-10^{1} \mu \mathrm{m}^{2}$ area of the sample, produce the higher irradiance than global illumination schemes $\left(10^{2}-10^{4} \mu \mathrm{m}^{2}\right)$ because the illuminating source is focused upon a significantly smaller area of the sample; the irradiance of line scanning would be in between those of point and global illumination schemes.

Spectral signal to noise ratios in Raman imaging can be improved by most of the coaddition schemes that have been introduced for FTIR imaging, albeit with appropriate modifications to the acquisition process. Spectra can be synthetically handled as in FTIR imaging, including the potential use of transformations, smoothing procedures, and factor analysis.

\subsubsection{Point mapping}

Point-mapping schemes involve the illumination of an individual point of the sample and concurrent detection of the spectral features of that point. In instruments containing a dispersive element, inelastically scattered lines are dispersed across only one component of the CCD. Rastering of the laser spot across the sample imparts spatial resolution. Although this method offers comparatively high laser spot power densities over other illumination schemes, the durations of sample movement and detector integration make this a very time consuming acquisition scheme. 


\subsubsection{Line Scanning}

The position of a laser spot on a sample can be controlled by modulating the angle at which the illuminating source enters objective lens. One rapidly oscillating mirror placed in the optical path can effectively raster the laser spot across one dimension of the sample plane. Uniformity of the laser intensity along the illuminated line can be achieved by use of a Powell lens placed in front of microscopic objective ${ }^{48}$. The laser power density at any point along the illuminated line is considerably lower than in pointmapping since the spot position oscillates in the sample plan. Both spatial and spectral features of scattered light can be retained upon projection across the detector plane ${ }^{49}$. Thus, one component of the FPA can impart spatial resolution for the illuminated line while the other component of the FPA imparts spectral resolution (Figure 1.3b).

This method offers a significant temporal advantage over point-mapping schemes by eliminating the need for sample rastering in one direction. The temporal resolution of line scanning schemes is, however, compromised by the need for longer detector integration times owing to the relatively low laser power densities at each position along the illuminated line.

\subsubsection{Global Illumination}

Spectral filters can be used to acquire global Raman images in the same way that they are used to acquire infrared images - two components of the FPA imparting two dimensions of spatial resolution. A bandpass filter is placed between the sample and detector to eliminate all but a selected range of scattered lines. There are a few phenomenological 
differences that confound the use of global Raman imaging. In terms of sample illumination, the power densities are thousands of times smaller than those in the point and line illumination schemes. Accordingly, detector integration times need to be considerably (essentially two orders of magnitude) longer than those for point illumination. Global illumination also raises the possibility of illuminating fluorescent impurities, the signal of which may overwhelm the intensity of inelastically scattered light from other spots in the sample. Furthermore, apertures of any kind cannot be used in the microscope's optical path, eliminating the potential for depth profiling.

The performance of a global Raman imaging instruments has been characterized in a number of studies. The spatial resolution of features extracted from of global and point acquisition schemes were consistent with one another ${ }^{50}$. It has also been demonstrated that spectral resolution can be effectively enhanced, despite relatively broad filter bandpasses, by forming component-specific images based on a band's center of mass rather than its intensity or position ${ }^{51}$.

\subsubsection{Materials Applications}

\subsubsection{Diffusion}

Most of the diffusion phenomena that have been studied by Raman imaging have been performed by confocal depth profiling. As mentioned above, the depth profiles must be handled cautiously due to refraction effects on the depth of field.

Heating of multilayer films above the $\mathrm{T}_{\mathrm{g}}$ of one component can promote interfacial diffusion of macromolecules. The diffusion process depends on the identity of 
the materials as well as the molecular weight of the material in each layer Diffusion of PMMA material into PVOH material has been analyzed by Raman depth profiling ${ }^{52}$. Interpenetrated regions of the system exhibited hydrogen bond, providing spectral evidence for interfacial diffusion. The diffusion of PMMA apparently slowed upon increase of the PMMA molecular weight due to the decrease of the diffusion coefficient with increasing chain length.

Silanes are frequently used to promote interfacial adhesion between organic and inorganic materials. The strength of adhesion typically relies on diffusion of the silane into the organic layer and the subsequent formation of an interpenetrated network. Raman depth profiles have shown that diffusion of the silanes into PVC depends on both the chemical composition of the silane material and the $T_{g}$ of the polymer ${ }^{53}$. Faster diffusion was observed for plasticized PVC materials that have a relatively low $\mathrm{T}_{\mathrm{g}}$; this result indicates that polymer chain mobility has a considerable influence on the penetrant diffusion process.

The identity of a polymer substrate can control the diffusion of species into that material. Accordingly, the identity of a catalyst support can impact the reaction rate in catalyst support resins. Raman imaging has allowed the simultaneous observation of both reagent uptake and conversion of 1,3 diisopropyl carbodiimide by supported 4cyanobenzoic acid resins ${ }^{54}$. The reagent's diffusion rate was faster than the conversion rate in a PS supported bead, leading to a homogeneous distributions of reagents across the bead profile. In contrast, for a TantaGel supported resin the conversion rate was faster than the diffusion of the reactant. This result reiterates the impact of substrate identity on diffusion processes and the importance of diffusion in catalyst support resins. 


\subsubsection{Processing Conditions}

Certain molecules may be loaded into polymer substrates to impart a desireable characteristic to the substrate, like coloration, adhesion or therapeutic activity. Loading can be accomplished by swelling the substrate with a solution that contains the species to be loaded. Confocal Raman microscopy has been used to assess the loading of an azo dye into the surface of a PET film ${ }^{55}$ and the loading of chlorinated polyolefins into a PP/ EPR blends ${ }^{56}$. Upon removal from the loading conditions, each system exhibited measurable increases in the depth of penetration over time by either the dye or the chlorinated polyolefin, respectively. The uptake of dye by PET followed a Fickian diffusion profile and the estimated diffusion coefficient of the dye was consistent with literature value of similar species ${ }^{55}$. The presence of solvent in the PP/ EPR blend system appeared to induce dissolution of the EPR domains during the loading process ${ }^{56}$.

The extent of loading of a molecule onto a substrate, and the associated depth profile, depends on a variety of factors including the duration of exposure to a loading mixture, the composition of the loading mixture, the system temperature, and the nature of the diffusant. Confocal Raman depth profiles have been used to asses the impact of all these factors on the diffusion of aminothiophenol into PVC ${ }^{57}$. Diffusion of the aminothiophenol species was followed by the substitution for chlorine atoms on the PVC backbone, so control over the diffusion process enabled the selective modification of the PVC surface. The highest surface selectivities in this study were observed for brief exposures to poor solvent systems at low temperature. 


\subsubsection{Chemical modification of substrates}

Polymer substrates are often activated or functionalized upon exposure to some extreme environmental conditions, like exposure to UV radiation or plasma. The material's chemical response to the environment often depends on the microscopic concentration of impurities or the chemical composition of a phase in the substrate. Microspectroscopy is ideal for studying the effects of local composition on chemical changes.

The role of residual Ziegler-Natta catalyst particles in the degradation of

polypropylene by photo-oxidation has been studied by Raman imaging ${ }^{58}$. While a variety of other analytical techniques can demonstrate the heterogeneous distribution of oxidative byproducts, Raman imaging identified an anti-correlation between the presence of catalyst species and oxidation byproducts. Polymer chains in the immediate proximity of the catalyst particles exhibited fewer oxidation by-products than the material distant from thee residual catalyst domains. The spectroscopic evidence supported the notion that catalyst particles can effectively reduce the oxidative species, but those oxidative species which diffuse away from the catalyst particles promote polymer degradation.

Grafting of macromolecular branches onto a substrate is a useful strategy for controlling a material's surface properties. Plasma treatment is one method used to generate grafting sites onto a preexisting polymer substrate. Raman imaging has been used to show that plasma treatment of a PP/ EPR blend produced grafting sites preferentially on EPR domains ${ }^{59}$. The results suggested that the EPR domains served as the grafting substrate because plasma-activated sites on the PP domains underwent a chain scission process which degraded the PP domain. 


\subsubsection{Phase/ Structural Transitions}

Morphological features of a polymer material are a product of both local chemical compositions and processing conditions. A number of polymers demonstrate semicrystalline tendencies; crystalline domains are microstructural features that have a foremost impact on material properties. Raman imaging is ideal for studying the effects of processing conditions on the development of microstructure because it offers quantitative chemical information of microscopic sample features. Raman imaging provides a route to understanding the complex relationships between composition, structure, and properties of polymer systems.

\subsection{Composition induced transitions}

Composition of a polymer blend often influences morphology, both in terms of miscibility and microstructure. Although two macromolecules may be immiscible and phase separate, trace amounts of either component are often dispersed in the complementary phase. Trace amounts of a foreign component can strongly influence the crystallization kinetics if the material in some phase is semicrystalline. For instance, two sorts of domains, spherulites and amorphous globules, can develop in phase separated polyether urethanes ${ }^{60}$, and the development of either domain is controlled by the local polymer composition. Composition-dependent crystallization kinetics have been supported by micro-Raman measurements in miscible and immiscible PPG/ polyester blends ${ }^{61}$. The results of this study quantitatively showed that low concentrations of a non-crystalline material (PPG) reduced the rate of crystallization in the semicrystalline 
polyester domains. Not so surprisingly, the development of crystallinity in miscible systems was considerably inhibited by the presence of PPG.

Branching of polymer chains can also impact the microstructure of a polymer blend. For instance, highly branched polyethylenes tend to be immiscible with linear polyethylenes despite the basically identical chemical nature of the two materials. Raman imaging of PE blends (linear mixed with branched) which were prepared under

different thermal histories, provided insight to the mechanism for PE phase separation ${ }^{62}$. Interestingly, the observed phase separation did not directly correlate with branch content, rather the mechanism of phase separation originated from nucleation and crystallization rate differences between the two blend components.

\subsection{Processing conditions}

Melt processing can effectively erase the thermal history of components in a material, and crystalline domains may form upon cooling from the melt. In the field of drug release, crystallinity often impacts the release profile for a particular formulation. Extruded mixtures of ibuprofen in a PVP matrix been characterized by Raman microscopy ${ }^{63}$. Spectral features across the sample indicated that the drug took an amorphous form which was homogeneously distributed across the formulation. Furthermore, those features did not change upon mild heating of the sample. This study demonstrated the application of Raman imaging for testing the stability of drug formulations.

Macromolecules at the surface of injection molded material have a different thermal history from those molecules in the middle of the material. The thermal 
conditions generate a structural gradient if the macromolecules have a semicrystalline tendency. Raman imaging methods have been used to identify crystallinity gradients in PET samples ${ }^{64}$ and gradients of domain size in PP/ PE blends ${ }^{65}$. Generally, molten material the contacts the surface of a mold cools quickly, allowing less time for crystallization or coalescence. Thus, molecules in the center of the material tend to display higher degrees of crystallinity or larger dispersed domains compared to skin material of the part. Additionally, polarized Raman measurements can provide information about the degree of orientation within a particular region ${ }^{65}$.

Macromolecules in the melt can be induced to nucleate on specific substrates even above the typical crystallization temperature. The formation of transcrystalline domains often occurs at an interface between a polymer melt and a fiber. Polarized Raman imaging is ideal for identifying the nature and orientation of transcrystalline domains. Raman imaging has demonstrated the formation transcrystalline domains of iPP in the presence of PET fibers ${ }^{66} 67$. The microscopic resolution of Raman imaging has enabled the visualization of lamellar branching as the transcrystalline domain extends away from the fiber.

\subsection{Stress induced}

Shear and tensile stress on polymers can promote orientation of the polymer chains in a sample; those actions can also induce crystallization and polymorphic transitions in semicrystalline polymers. Raman imaging has been used to investigate the mechanism for crystalline phase transitions upon plastic deformation of a Nylon 6 sample ${ }^{68}$. Nylon 6 is capable of forming three crystalline structures $(\alpha, \beta$, and $\gamma)$, and each phase can be 
distinguished by its Raman spectra. Plastic deformation of a solvent cast sample produced an increased amount of $\alpha$-phase and decreased amounts of $\beta$ and $\gamma$ phases. The mechanism for the crystalline transition involved the formation of fibrils and subsequent slipping and reorientation of lamellae as monitored by AFM, and supported by Raman.

\subsection{Other Raman and FTIR Imaging Applications}

The power of spectroscopic imaging lies in the development of chemical contrast between different regions of the sample plane. This contrast mechanism has been successfully applied to a variety of disciplines other than polymer science and engineering.

\subsubsection{Online Applications}

The most important factor for applying hyperspectral imaging to online applications is the sampling speed, temporal resolution. In terms of sample preparation, scattering and reflection methods (Raman, ATR and NIR) hold a considerable advantage over transmission methods. Most often, only a few specific spectral bands are needed to monitor a process or an object's identity. Thus, global imaging methods based on spectral filtration hold the highest potential for online applications. Near-IR imaging has been used in conjunction with multivariate statistical analysis to discriminate different plastic objects in solid municipal waste ${ }^{69} 70$.

\subsubsection{Parallel Detection Techniques}

The sample plane can be divided into defined regions, termed chambers, which can serve as miniature reactors. This set-up enables a variety of conditions to be investigated in 
parallel. Sample coaddition can be applied if a single chamber covers several pixels in the array plane.

A rapid scanning FTIR imaging apparatus has been used for the parallel evaluation of catalysts used for propene combustion ${ }^{71}$, carbon monoxide oxidation ${ }^{72}$, and decomposition of ammonia ${ }^{73}$. An ATR imaging apparatus has been used to evaluate the uptake of atmospheric moisture by a series of drug formulations in parallel ${ }^{74}$; the use of an ATR objective enabled the atmosphere on one side of the samples to be controlled. Arrays of tissue samples have also been imaged and spectroscopically classified in parallel $^{75}$.

\subsubsection{Physiological Applications}

Chemical contrast is an appealing approach for characterizing histological samples because it averts the use of contrast-generating stains. Although many physiological samples contain water, the resulting infrared absorbances can be reduced through the use of ATR objectives, dried samples, or clever preprocessing.

Raman depth profiles have demonstrated the uptake of a photosensitizer for photodynamic therapy in different cell lines ${ }^{76}$; the use of long incident wavelength laser light prevented activation of the photosensitive agent, providing a unique advantage over fluorescence microscopy. Raman depth profiling has also been used to investigate the location of small molecules in layers of skin.

FTIR imaging has provided insight to bone mineral composition and collagen characteristics under several conditions including PHT treatment ${ }^{77}$, rhPTH treatment ${ }^{78}$ ALN treatment ${ }^{79}$, BMP- 6 treatment ${ }^{80}$, growth factor deficiencies ${ }^{81}$, TNAP deficiency ${ }^{82}$ 
and the conditions of rickets and osteomalacia ${ }^{83}$. FTIR imaging has also provided insight to the chemical composition distributions of artherosclerotic blood vessels ${ }^{84}$ and heart tissue in myocarditis ${ }^{85}$. The distribution of proteoglycan in engineered cartilage has been monitored with FTIR imaging ${ }^{86}$.

\subsection{Summary}

Array detectors are an essential feature of Raman and infrared imaging techniques. Advances in detector technology are responsible for decreasing image acquisition times compared to early image acquisition methods. Fast image acquisition has enabled in situ monitoring of processes like diffusion, dissolution, and a variety of phase transitions. FTIR imaging has provided powerful insights to the topics of polymer dissolution and controlled release. The applications of FTIR imaging are becoming more robust with the introduction of continuous scanning spectrometers and ATR objectives. Of the three approaches to acquiring Raman images (global illumination, point mapping, and line scanning), the line scanning approach holds the highest potential for analyzing dynamic systems because it provides spatial and spectral information in parallel. Confocal optical configurations have enabled the acquisition of Raman depth profiles, which are excellent for studying diffusion processes. Beyond materials science, Raman and infrared imaging methods have been applied to online applications, parallel investigations, and histological studies. The future of Raman and infrared imaging techniques lies in expanding the base of applications and decreasing the image acquisition time. 


\subsection{Works Cited}

1. J. L. Koenig, Microspectroscopic Imaging of Polymers;(American Chemical Society: Washington, DC, 1998).

2. J. M. Harnly and R. E. Fields. Applied Spectroscopy, 51, 334 (1997).

3. E. N. Lewis, P. J. Treado, R. C. Reeder, G. M. Story, A. E. Dowrey, C. Marcott and I. W. Levin. Analytical Chemistry, 67, 3377 (1995).

4. J. Sommer, L. G. Tisinger, C. Marcott and G. M. Story. Applied Spectroscopy, 55, 252 (2001).

5. K. L. A. Chan and S. G. Kazarian. Applied Spectroscopy, 57, 381 (2003).

6. R. Bhargava, M. D. Schaeberle, D. C. Fernandez and I. W. Levin. Applied Spectroscopy, 55, 1079 (2001).

7. S. W. Huffman, R. Bhargava and I. W. Levin. Applied Spectroscopy, 56, 965 (2002).

8. C. A. Coutts-Lendon, N. A. Wright, E. V. Mieso and J. L. Koenig. Journal of Controlled Release, 93, 223 (2003).

9. E. N. Lewis, A. M. Gorbach, C. Marcott and I. W. Levin. Applied Spectroscopy, 50, $263(1996)$.

10. P. Dumas, N. Jamin, J. L. Teillaud, L. M. Miller and B. Beccard. Faraday Discussions, 126, 289 (2003).

11. L. M. Miller and R. J. Smith. Vibrational Spectroscopy, 38, 237 (2005).

12. R. Bhargava and I. W. Levin. Analytical Chemistry, 73, 5157 (2001).

13. C. M. Snively and J. L. Koenig. Applied Spectroscopy, 53, 170 (1999). 
14. R. Bhargava and I. W. Levin. Analytical Chemistry, 74, 1429 (2002).

15. R. Bhargava, D. C. Fernandez, M. D. Schaeberle and I. W. Levin. Applied Spectroscopy, 55, 1580 (2001).

16. R. Bhargava, T. Ribar and J. L. Koenig. Applied Spectroscopy, 53, 1313 (1999).

17. R. Bhargava, S.-Q. Wang and J. L. Koenig. Applied Spectroscopy, 54, 1690 (2000).

18. R. Bhargava, S.-Q. Wang and J. L. Koenig. Applied Spectroscopy, 54, 486 (2000).

19. J. P. Bobiak and J. L. Koenig. Journal of Controlled Release, 106, 329 (2005).

20. C. M. Snively and J. L. Koenig. Journal of Polymer Science, Part B: Polymer Physics, 37, 2261 (1999).

21. C. Snively and J. Koenig. Macromolecules, 31, 3753 (1998).

22. D. W. Rafferty and J. L. Koenig. Journal of Controlled Release, 83, 29 (2002).

23. K. L. A. Chan and S. G. Kazarian. Vibrational Spectroscopy, 35, 45 (2004).

24. G.-Y. Li and J. L. Koenig. Applied Spectroscopy, 56, 1390 (2002).

25. G.-Y. Li and J. L. Koenig. Polymer Degradation and Stability, 81, 377 (2003).

26. J. van der Weerd and S. G. Kazarian. Journal of Controlled Release, 98, 295 (2004).

27. S. G. Kazarian and A. K. L. Chan. Macromolecules, 36, 9866 (2003).

28. B. A. Miller-Chou and J. L. Koenig. Progress in Polymer Science, 28, 1223 (2003).

29. T. Ribar, R. Bhargava and J. L. Koenig. Macromolecules, 33, 8842 (2000).

30. T. Ribar, J. L. Koenig and R. Bhargava. Macromolecules, 34, 8340 (2001).

31. J. Gonzalez-Benito and J. L. Koenig. Macromolecules, 35, 7361 (2002). 
32. B. A. Miller-Chou and J. L. Koenig. Macromolecules, 35, 440 (2002).

33. J. P. Bobiak and J. L. Koenig. Applied Spectroscopy, 58, 1141 (2004).

34. S. J. Oh and J. L. Koenig. Analytical Chemistry, 70, 1768 (1998).

35. D. W. Rafferty, J. L. Koenig, G. Magyar and J. L. West. Applied Spectroscopy, 56,284 (2002).

36. C. M. Snively and J. L. Koenig. Journal of Polymer Science, Part B: Polymer Physics, 37, 2353 (1999).

37. E. Shin and J. L. Koenig. Journal of Adhesion, 78, 877 (2002).

38. J. Gonzalez-Benito. Journal of Colloid and Interface Science, 267, 326 (2003).

39. R. Bhargava, S.-Q. Wang and J. L. Koenig. Macromolecules, 32, 2748 (1999).

40. O. S. Fleming, K. L. A. Chan and S. G. Kazarian. Vibrational Spectroscopy, 35, 3 (2004).

41. S. G. Kazarian, K. L. A. Chan, V. Maquet and A. R. Boccaccini. Biomaterials, 25, 3931 (2004).

42. S. G. Kazarian and K. L. A. Chan. Macromolecules, 37, 579 (2004).

43. R. Bhargava, S.-Q. Wang and J. L. Koenig. Applied Spectroscopy, 52, 323 (1998).

44. P. Tomba and G. Eli. Applied Spectroscopy, 57, 920 (2003).

45. N. J. Everall. Applied Spectroscopy, 54, 773 (2000).

46. N. J. Everall. Applied Spectroscopy, 54, 1515 (2000).

47. F. Adar, C. Naudin, A. Whitley and R. Bodnar. Applied Spectroscopy, 58, 1136 (2004).

48. K. A. Christensen and M. D. Morris. Applied Spectroscopy, 52, 1145 (1998). 
49. M. Bowden, D. J. Gardiner, G. Rice and D. L. Gerrard. Journal of Raman Spectroscopy, 21, 37 (1990).

50. L. Markwort, B. Kip, E. Da Silva and B. Roussel. Applied Spectroscopy, 49, 1411 (1995).

51. M. D. Schaeberle, D. D. Tuschel and P. J. Treado. Applied Spectroscopy, 55, 257 (2001).

52. S. Hajatdoost and J. Yarwood. Applied Spectroscopy, 50, 558 (1996).

53. P. Eaton, P. Holmes and J. Yarwood. Applied Spectroscopy, 54, 508 (2000).

54. J. Kress, R. Zanaletti, A. Rose, J. G. Frey, W. S. Brocklesby, M. Ladlow and M. Bradley. Journal of Combinatorial Chemistry, 5, 28 (2003).

55. O. S. Fleming, F. Stepanek and S. G. Kazarian. Macromolecular Chemistry and Physics, 206, 1077 (2005).

56. H. R. Morris, B. Munroe, R. A. Ryntz and P. J. Treado. Langmuir, 14, 2426 (1998).

57. J. Sacristan, C. Mijangos, H. Reinecke, S. Spells and J. Yarwood. Macromolecules, 33, 6134 (2000).

58. I. Blakey and G. A. George. Polymer Degradation and Stability, 70, 269 (2000).

59. I. Keen, G. A. George and P. M. Fredericks. Journal of Applied Polymer Science, 88, 1643 (2003).

60. H. Janik, B. Pays and Z. S. Petrovic. Macromolecular Rapid Communications, 24, 265 (2003).

61. T. Hashida, Y. G. Jeong, Y. Hua, S. L. Hsu and C. W. Paul. Macromolecules, 38, $2876(2005)$ 
62. R. L. Morgan, M. J. Hill, P. J. Barham, A. van der Pol, B. J. Kip, R. Ottjes and J. van Ruiten. Polymer, 42, 2121 (2001).

63. J. Breitenbach, W. Schrof and J. Neumann. Pharmaceutical Research, 16, 1109 (1999).

64. W. B. Liau and C. F. Chang. Journal of Applied Polymer Science, 76, 1627 (2000).

65. S. L. Quintana, P. Schmidt, J. Dybal, J. Kratochvl, J. M. Pastor and J. C. Merino. Polymer, 43, 5187 (2002).

66. M. R. Fernandez, J. C. Merino, M. I. Gobernado-Mitre and J. M. Pastor. Applied Spectroscopy, 54, 1105 (2000).

67. J. C. Merino, M. R. Fernandez and J. M. Pastor. Macromolecular Symposia, 168, 55 (2001).

68. V. Ferreiro, C. Depecker, J. Laureyns and G. Coulon. Polymer, 45, 6013 (2004).

69. W. H. A. M. Van Den Broek, D. Wienke, W. J. Melssen, R. Feldhoff, T. HuthFehre, T. Kantimm and L. M. C. Buydens. Applied Spectroscopy, 51, 856 (1997).

70. A. Kulcke, C. Gurschler, G. Spock, R. Leitner and M. Kraft. Journal of Near Infrared Spectroscopy, 11, 71 (2003).

71. C. M. Snively, G. Oskarsdottir and J. Lauterbach. Angewandte Chemie, International Edition, 40, 3028 (2001).

72. C. M. Snively, G. Oskarsdottir and J. Lauterbach. Catalysis Today, 67, 357 (2001).

73. R. J. Hendershot, R. Vijay, B. J. Feist, C. M. Snively and J. Lauterbach. Measurement Science and Technology, 16, 302 (2005). 
74. K. L. A. Chan and S. G. Kazarian. Journal of Combinatorial Chemistry, 7, 185 (2005).

75. D. C. Fernandez, R. Bhargava, S. M. Hewitt and I. W. Levin. Nature Biotechnology, 23, 469 (2005).

76. T. L. Freeman, S. E. Cope, M. R. Stringer, J. E. Cruse-Sawyer, S. B. Brown, D. N. Batchelder and K. Birbeck. Applied Spectroscopy, 52, 1257 (1998).

77. E. Paschalis, P., E. Glass, V., D. Donley, W. and E. Eriksen, F. Journal of clinical endocrinology and metabolism FIELD Publication Date:2005 Aug, 90, 4644

78. E. P. Paschalis, D. B. Burr, R. Mendelsohn, J. M. Hock and A. L. Boskey. Journal of Bone and Mineral Research, 18, 769 (2003).

79. N. P. Camacho, P. Carroll and C. L. Raggio. Calcified Tissue International, 72, 604 (2003).

80. A. L. Boskey, E. P. Paschalis, I. Binderman and S. B. Doty. Journal of Cellular Biochemistry, 84, 509 (2001).

81. E. Atti, S. Gomez, S. M. Wahl, R. Mendelsohn, E. Paschalis and A. L. Boskey. Bone (New York, NY, United States), 31, 675 (2002).

82. H. C. Anderson, J. B. Sipe, L. Hessle, R. Dhamyamraju, E. Atti, N. P. Camacho and J. L. Millan. American Journal of Pathology, 164, 841 (2004).

83. D. Faibish, A. Gomes, G. Boivin, I. Binderman and A. Boskey. Bone (San Diego, CA, United States), 36, 6 (2005).

84. C. S. Colley, S. G. Kazarian, P. D. Weinberg and M. J. Lever. Biopolymers, 74, 328 (2004). 
85. Q. Wang, W. Sanad, L. M. Miller, A. Voigt, K. Klingel, R. Kandolf, K. Stangl and G. Baumann. Vibrational Spectroscopy, 38, 217 (2005).

86. P. Spencer, Y. Wang, J. L. Katz and A. Misra. Journal of Biomedical Optics, 10, 031104/1 (2005). 


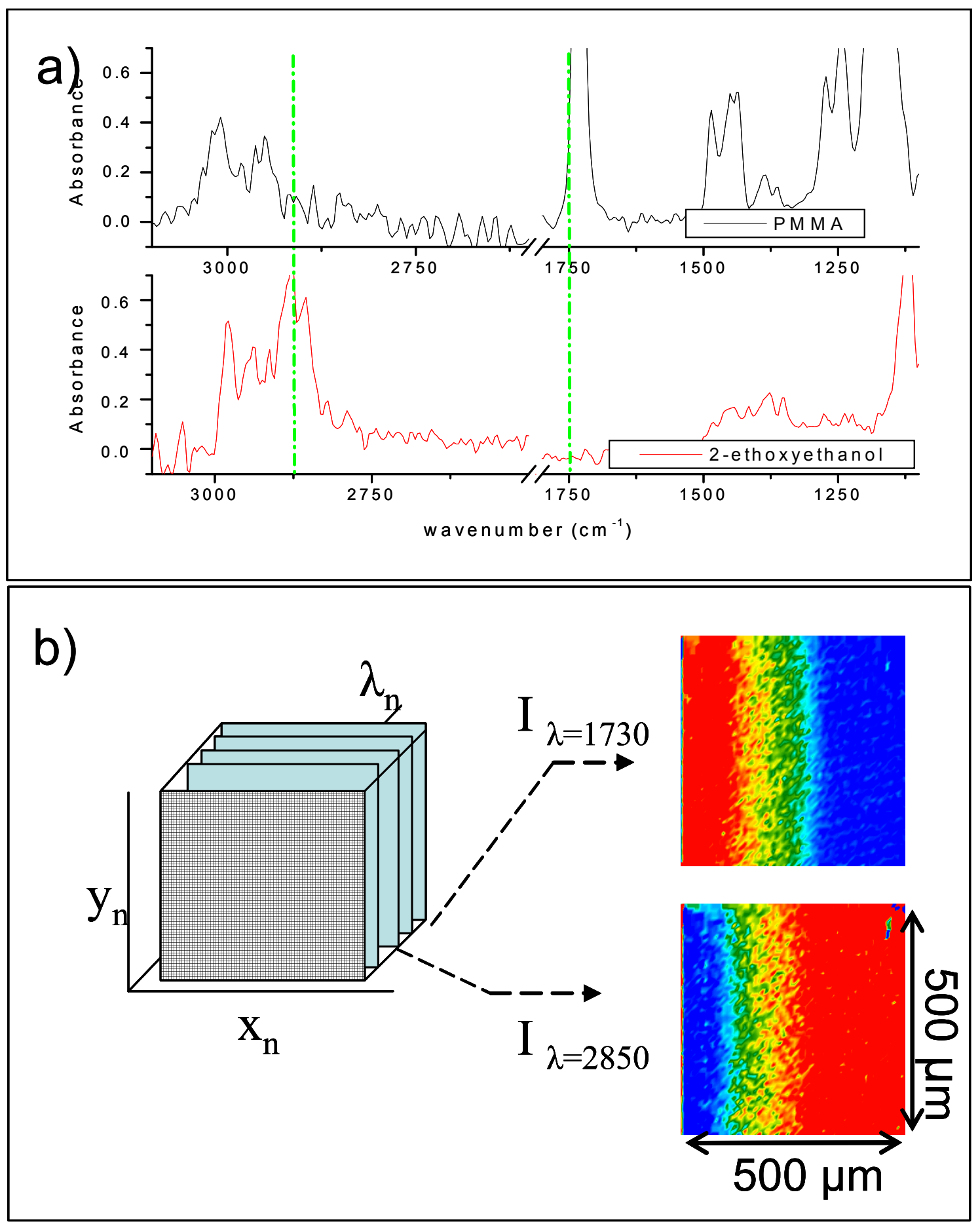

Figure 1.1. The mechanism for chemical contrast displayed for a PMMA/ 2ethoxyethanol interface. 


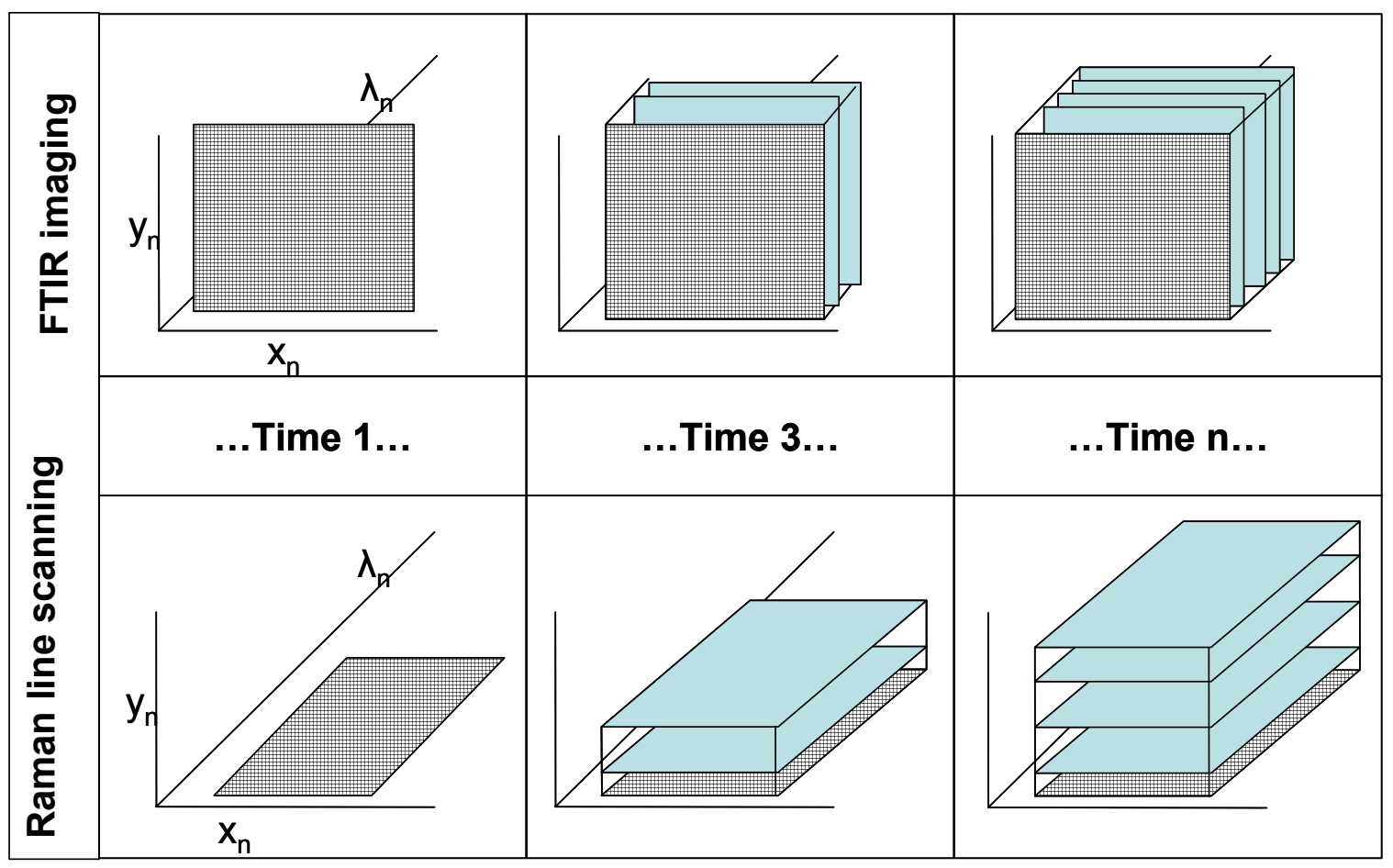

Figure 1.2. Development of a hyperspectral cube by FTIR imaging and Raman line scanning. In FTIR imaging, steps of the spectrometer develops the wavelength $(\lambda)$ dimension. In Raman line scanning, stage rastering develops one spatial (y) dimension. 


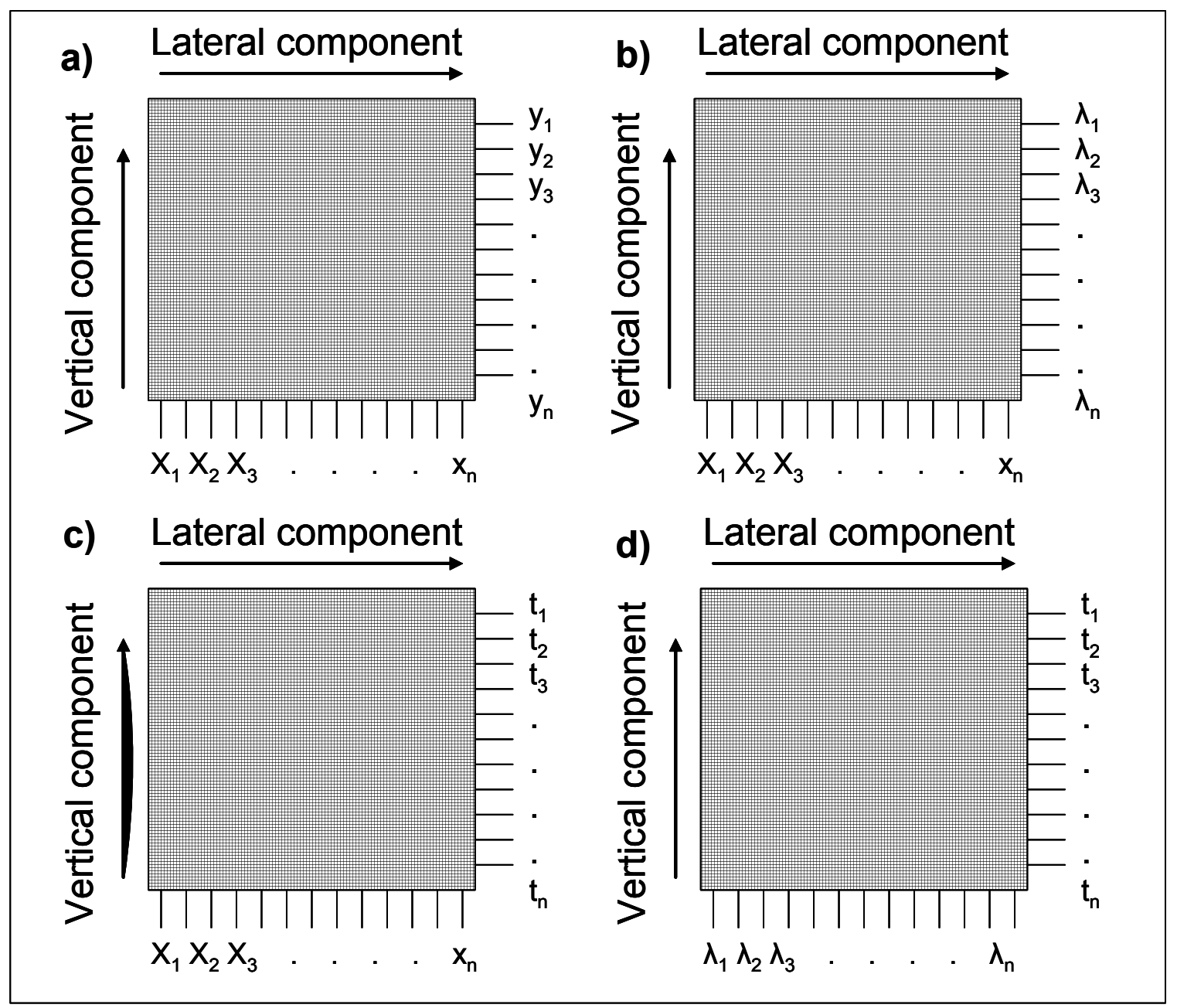

Figure 1.3. Schematic illustration of various array detector uses. 


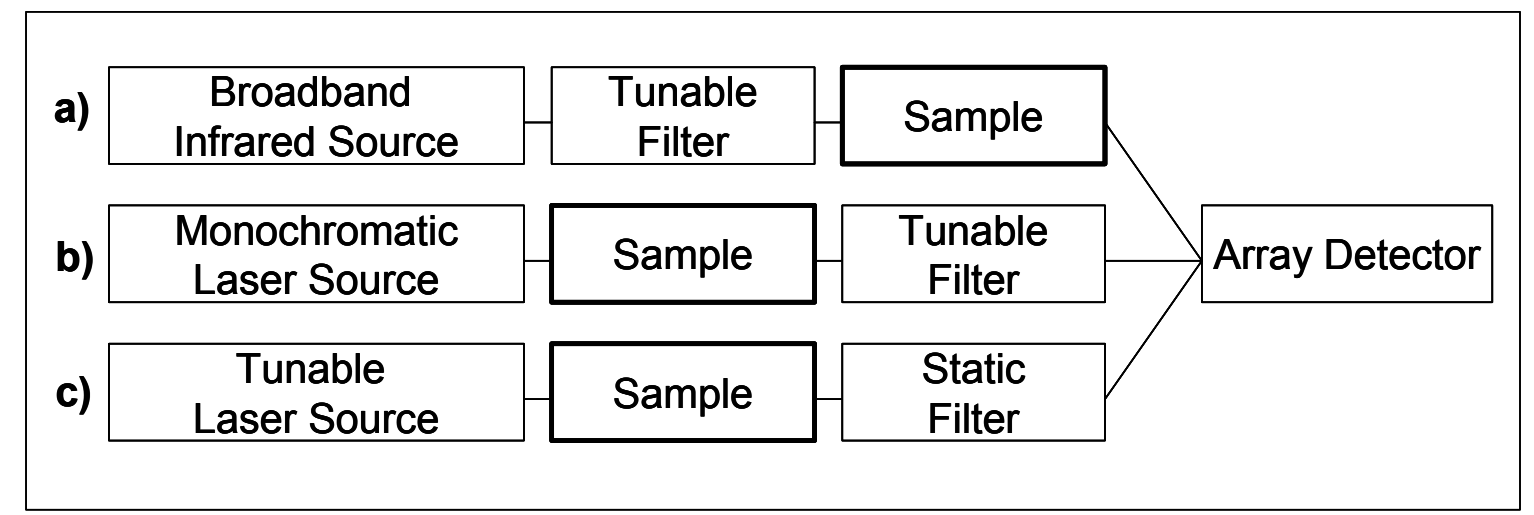

Figure 1.4. Schematic diagram of filtration-based infrared (a) and Raman (b, c) global imaging schemes. 


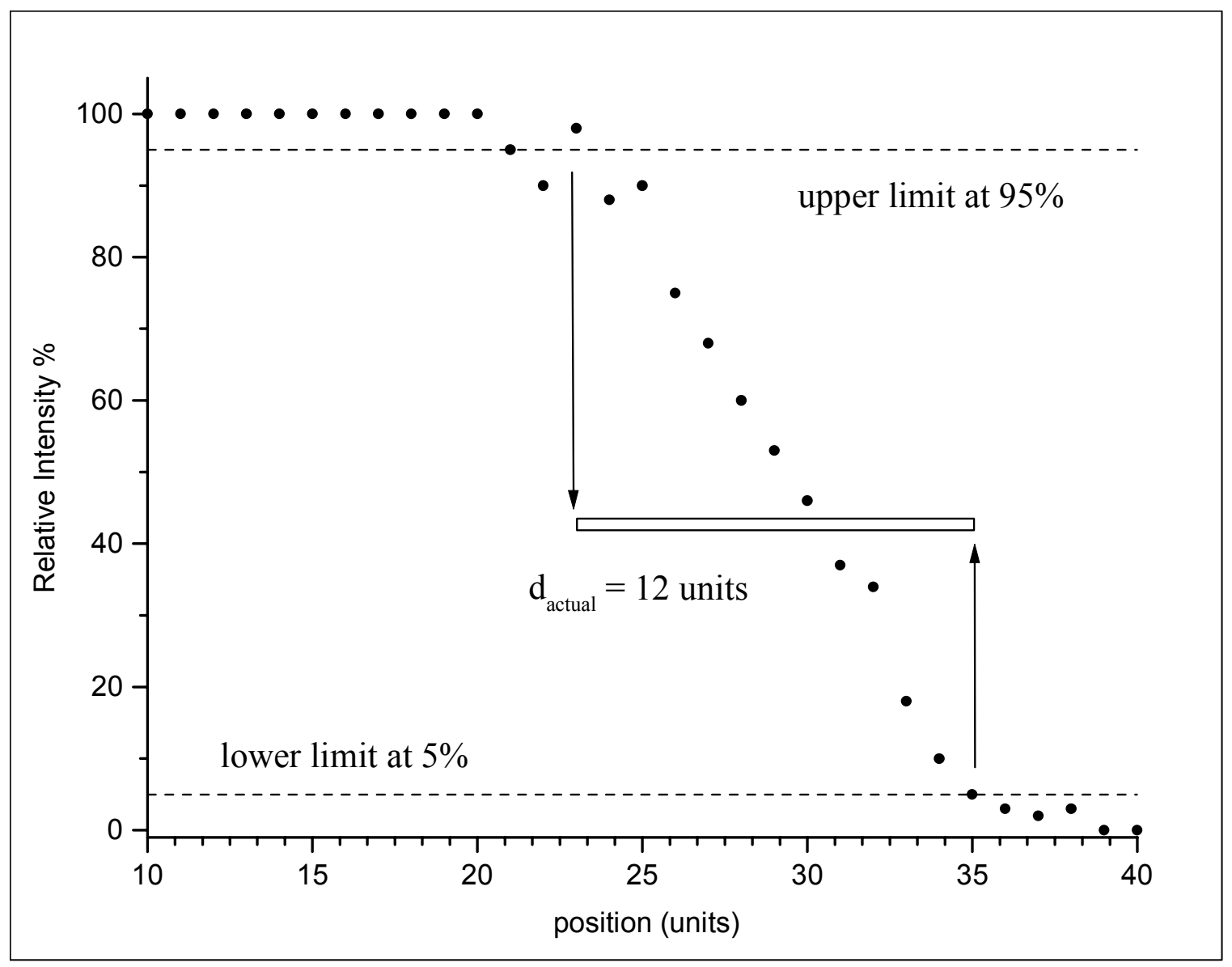

Figure 1.5. Illustration of the $d_{\text {actual }}$ value for determination of the degradation factor. 


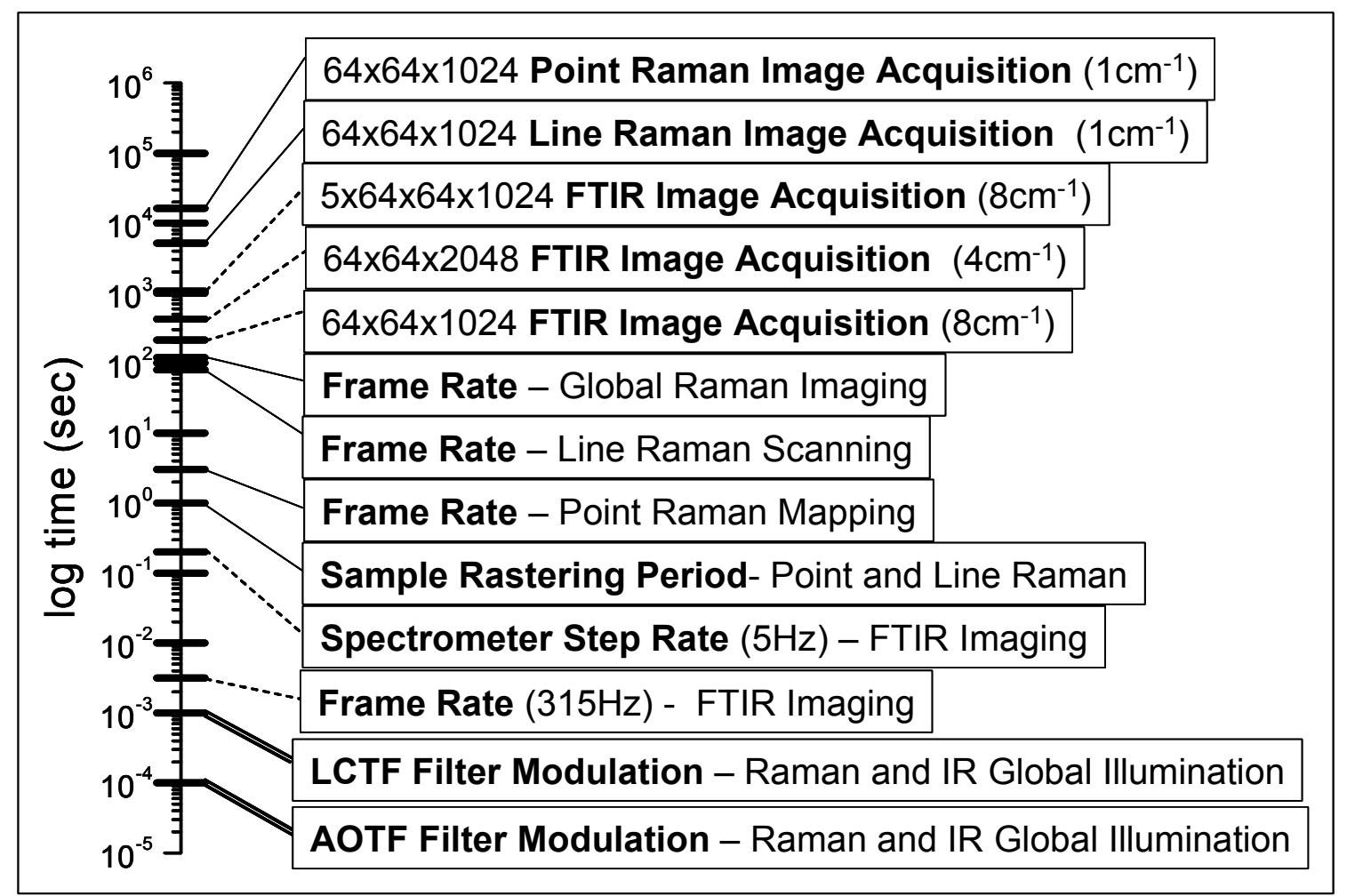

Figure 1.6. Approximate duration of instrumental processes while acquiring Infrared (dashed lines) and Raman (solid lines) Images. Processes shared by some techniques are indicated by double solid lines. 


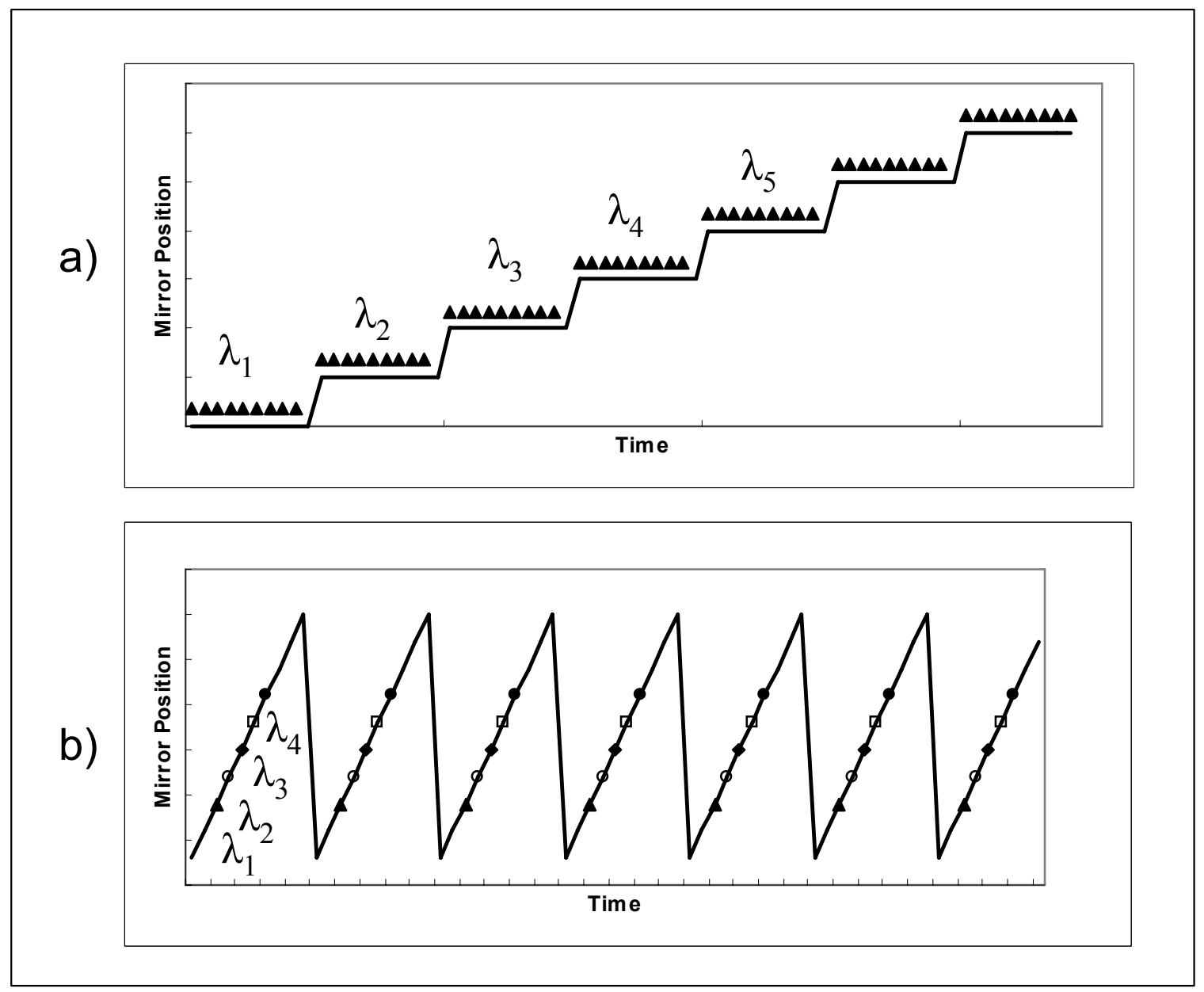

Figure 1.7. Schematic diagrams for step-scanning acquisition schemes (a) and continuous-scanning acquisition schemes (b). 


\begin{tabular}{|c|c|c|}
\hline $\begin{array}{l}\text { Resolution } \\
\text { Dimension }\end{array}$ & FTIR Imaging & $\begin{array}{c}\text { Raman Line } \\
\text { Scanning }\end{array}$ \\
\hline Position (x) & Array detector $(\mathrm{x})$ & $\begin{array}{l}\text { Modulation of laser spot } \\
(\mathrm{x}) / \text { Array detector }(\mathrm{x})\end{array}$ \\
\hline Position (y) & Array detector (y) & Sample rastering $(\mathrm{y})$ \\
\hline Frequency $(\lambda)$ & $\begin{array}{c}\text { Interferrometer } \\
\text { movement }\end{array}$ & $\begin{array}{l}\text { Dispersive Element/ } \\
\text { Array detector }(\mathrm{y})\end{array}$ \\
\hline $\begin{array}{l}\text { Spectral width limited } \\
\text { by: }\end{array}$ & $\begin{array}{l}\text { Interferrometer } \\
\text { movement }\end{array}$ & $\begin{array}{l}\text { Dispersive element/ } \\
\text { Detector dimensions }\end{array}$ \\
\hline $\begin{array}{l}\text { Spectral SNR } \\
\text { improved by: }\end{array}$ & $\begin{array}{l}\text { Data filtering and } \\
\text { coaddition }\end{array}$ & $\begin{array}{l}\text { Data filtering and } \\
\text { coaddition }\end{array}$ \\
\hline $\begin{array}{l}\text { Spatial resolution } \\
\text { limited by: }\end{array}$ & Diffraction & Diffraction \\
\hline
\end{tabular}

Table 1.A. Origins of resolution dimensions for spectral imaging. 


\section{FTIR Imaging of Stereoregular Poly(methyl methacrylate) Dissolution}

\subsection{Abstract}

FTIR imaging was used to study dissolution of stereoregular poly (methyl methacrylate) (PMMA) films in toluene, benzene, chloroform, acetic acid and 2-ethoxyethanol. Images of the polymer-solvent interface showed that syndiotactic (sPMMA), atactic (aPMMA) and isotactic (iPMMA) samples dissolved in acetic acid and chloroform; only iPMMA dissolved in benzene, toluene and 2-ethoxyethanol. Concentration profiles allowed quantitative comparison of each polymer-solvent system. In the cases of chloroform and acetic acid, the rate of polymer dissolution increased with increasing isotacticity. Dissolution of PMMA in chloroform was observed to coincide with development of strong polymer-solvent interactions. The results of systems containing toluene, benzene and 2-ethoxyethanol reflect kinetic effects on dissolution, including solvent size and stiffness of the polymer backbone.

\subsection{Introduction}

Numerous commercial processes including lithography, membrane and coatings, recycling and drug delivery rely on the dissolution of polymers. The polymer dissolution process differs from that of low molecular weight compounds due to polymer

characteristics like molecular weight ${ }^{1,2,34}$, composition and distribution of monomers ${ }^{5}$, polydispersity $^{1,6}$, morphology $^{7}$, processing history $^{1}$ and stereoregularity ${ }^{8,9}$.

Stereoregularity provides an interesting avenue to tailor the dissolution process, among other properties, of polymers without altering the chemical identity. 
Stereoregularity influences flexibility and conformation of a polymer backbone. In the case of PMMA, tacticity manifests itself in different glass transition temperatures for isotactic PMMA $\left(40^{\circ} \mathrm{C}\right)$ and syndiotactic PMMA $\left(105^{\circ} \mathrm{C}\right)^{10}$. Differences in the mechanical ${ }^{11}$ and permeation ${ }^{12}$ properties of PMMA stereoisomers have been studied. Investigations of stereoregular PMMA in blending ${ }^{13,14,15,16,17 \text { 18, 19, } 20 \text { and casting }}{ }^{21,22,23}$ applications have illustrated the dependence of blend or film properties on both tacticity of the PMMA and solvent used for a particular application. Eased flexibility of an iPMMA backbone ultimately allows more contacts with interacting species, on a surface or in solution, compared to sPMMA.

This work focuses on the dissolution of various PMMA films by five solvents at room temperature. Uberreiter was one of the first researchers to model the dissolution process of glassy polymers ${ }^{6}$ and several precise mathematical models have developed since $^{7,24,25,26,27,28}$. Polymer dissolution has recently been reviewed by Miller-Chou and Koenig $^{29}$. Many methods have been used to study polymer dissolution including, laser interferrometry $^{30,31}$, ellispometry, steady state fluorescence ${ }^{3,32,33,34}$, optical microscopy ${ }^{5,}$ 35 , and FTIR imaging $2,36,37,38,39,40$. Of these, only FTIR imaging provides specific chemical information with both spatial and temporal resolution.

FTIR imaging has been used in this research group to study dynamic polymer systems, including polymer crystallization ${ }^{41}$, liquid crystal diffusion ${ }^{42,43}$, and dissolution of polymers in various environments ${ }^{2,36,37,38,39,40}$. Rafferty and Koenig studied selective dissolution of systems for drug delivery ${ }^{39}$. Ribar et al. and Miller-Chou and Koenig studied the effects of solvent mixtures on poly ( $\alpha$-methyl styrene) with varying molecular weight $^{37,38,40}$. And, most relevant to this work, Gonzalez-Benito and Koenig. studied 
dissolution of PMMA in cosolvent systems ${ }^{36}$. FTIR imaging is employed in this work to compare the dissolution process for three tacticities of PMMA in five different solvents. For a given solvent, different dissolution behavior among the PMMA samples will reflect the effect of backbone conformation. For a given tacticity of PMMA, dissolution with different solvents will reflect the effects of solvent size and interactions between polymer and solvent.

\subsection{Materials and Methods}

All solvents were reagent grade obtained from Aldrich and used as received. PMMA samples were obtained from Polymer Source, Toronto and used as received. Isotactic PMMA had $>98 \%$ iso content, $\mathrm{M}_{\mathrm{w}}$ of 43500 , and PDI of 1.13 . The syndiotactic PMMA sample had $79 \%$ syndio content, $\mathrm{M}_{\mathrm{w}}$ of 38500 , and PI of 1.04 . The atactic PMMA sample had $\mathrm{M}_{\mathrm{w}}$ of 8400 and PDI of 1.32. Solutions of $10 \%$ wt PMMA in chloroform were cast onto $\mathrm{CaF}_{2}$ slides and left in room conditions for 30 minutes. A sandwich was created by adding $10 \mu \mathrm{m}$ spacers and another $\mathrm{CaF}_{2}$ slide to the top of the cast film, then clamping the sample to assure good contact between the $\mathrm{CaF}_{2}$ plates and the polymer. Samples were heated at $175^{\circ} \mathrm{C}$ for 3 hours, cooled and stored in a dessicator at room temperature until use.

Dissolution studies applied the contact method in which solvent was delivered to the polymer interface by capillary action ${ }^{44}$. FTIR images of the polymer-solvent interface were collected in 3-6 minute intervals for up to 60 minutes. Solvent was added as necessary to replenish that which had evaporated. Table I lists some characteristics of 
the solvents tested ${ }^{45}$, including the distance of the solubility parameter from that of $\mathrm{PMMA}^{46}$.

FTIR images were collected using a Digilab Stingray which consists of an FTS 6000 step-scan interferometer coupled to a UMA 500 microscope accessory and a Santa Barbara focal plane array of 64x64 mercury-cadium-telluride elements. Each image represents a 500x500 $\mu \mathrm{m}$ area, providing a spatial resolution of approximately $8 \mu \mathrm{m}$. A long pass filter was used to eliminate unwanted wavelengths. A stepping rate of $5 \mathrm{~Hz}$ was used for the interferometer. A spectral resolution of $8 \mathrm{~cm}^{-1}$ was used and signal to noise ratio was increased by averaging 20 camera images per interferometer step. The resulting data cube was processed using Win IR Pro 3.3 and absorbance profiles were generated using Environment for Visualizing Images (ENVI) 4.0. Signal to noise ratio of absorbance profiles was increased by averaging the spectral data from a cross section of a particular image.

\subsection{Results and Discussion}

FTIR images of the polymer-solvent interface show differences for each solvent - PMMA combination. False-color images were generated by selecting an absorbance band present in only one component of the system, then plotting the peak height of that band for each pixel of the detector. In the system containing iPMMA and benzene, for instance, polymer images were generated by plotting the absorbance of the edge of the PMMA carbonyl band at $1755 \mathrm{~cm}^{-1}$ and benzene images were generated by plotting the band at $1478 \mathrm{~cm}^{-1}$. Figure 2.1 shows the iPMMA-benzene interface after different times of exposure to solvent. The uniform recess of the bulk polymer front, as well as the presence of carbonyl absorbance on the solvent side of the polymer front, shows that 
polymer molecules had entered the bulk solvent. Systems containing benzene with aPMMA or sPMMA showed eruption of polymer fragments, as opposed to uniform recession, shown in Figure 2.2. Eruption of the aPMMA fragments during dissolution are thought to originate from enhanced solvent migration and subsequent stress build up within local regions of the bulk polymer ${ }^{6,9}$ and has been observed in other FTIR imaging work $^{40}$.

The spatial distribution of chemical species over time was analyzed by using concentration profiles generated from a portion of the FTIR images. Profiles were generated by plotting the peak height of a selected absorbance band across an entire row of pixels. The term concentration profiles will be used synonymously with absorbance profiles. Figure 2.3 shows the normalized concentration profile for the iPMMA-benzene system over time. The profile extracted at 2 minutes shows that a small amount of benzene had penetrated the outermost layer of polymer.

Figure 2.4 and Figure 2.5 show FTIR images and normalized absorbance profiles of two absorbance bands for iPMMA during exposure to 2-ethoxyethanol. Profiles at $1750 \mathrm{~cm}^{-1}$ and $2857 \mathrm{~cm}^{-1}$ show a plateau representing a region of swollen polymer. The carbonyl band intensity at $1705 \mathrm{~cm}^{-1}$ represents intermolecular interactions between the carbonyl group of PMMA and hydroxyl group of 2-ethoxyethanol. Polymer- solvent interactions exist only in the penetrated or swollen region of the polymer, thus the profile of interacting carbonyl species does not show a distinct plateau. A similar carbonyl shift was observed by Benito-Gonzalez in FTIR imaging of cosolvent systems containing methanol $^{36}$. 
Three stages of the dissolution process are illustrated from the iPMMA systems containing benzene and 2-ethoxyethanol, corresponding to Ueberreiter's layer model for polymer dissoultion ${ }^{6}$. Figure 2.1 and Figure 2.3 show that outermost portion of the bulk iPMMA contains a small amount of penetrating benzene. Solvent molecules act to ease segment mobility in the penetrated polymer in the next stage of dissolution, generating an intermediate layer containing both solvent and polymer. This intermediate layer can be seen to grow with exposure time in the iPMMA-2-ethoxyethanol system shown in Figure 2.4. In the case of benzene, the swelling and dissolution of iPMMA were indistinguishable within the temporal resolution of the experiments. The final step of dissolution is disentanglement of the polymer molecules, which is represented by the recession of the polymer front in Figure 2.1.

Observations of these dissolution experiments must be related to material properties. Work toward predicting the diffusion of penetrants in polyacrylates has itself resulted in a number of theoretical models $\mathrm{s}^{46,47,48} 49,50,51$, which typically include factors for free volume and $\mathrm{T}_{\mathrm{g}}$ of the polymer, volume of solvent molecules, and thermodynamic compatibility between polymer and solvent. Models for amorphous polymer dissolution typically address solvent penetration, polymer swelling and chain disentanglement via the diffusion coefficients for solvent $\left(D_{\mathrm{s}}\right)$ and polymer $\left(D_{\mathrm{p}}\right)$, respectively. Kinematic models for the recess of polymer fronts have been reviewed elsewhere ${ }^{9,24,26,27,29}$. As a means of illustration, a continuum approach to the modeling can provide insight to the results in this investigation. The movement of the polymer front over time $(d S / d t)$ is related to the diffusion coefficients of the polymer and solvent by ${ }^{52}$ : 


$$
\frac{d S}{d t}=\left(D_{12} \frac{d v_{2}}{d x}\right)^{-}-\frac{D_{p}}{v_{1}^{-}}\left(\frac{d v_{2}}{d x}\right)^{+}, S(t=0)=0
$$

Where $S$ is position of the glassy-swollen polymer interface, $t$ is time, $x$ is distance, $v_{2}$ is the volume fraction of polymer in the swollen region of polymer, $v_{1}$ is the volume fraction of the solvent in the solvent-penetrated region of polymer, $\mathrm{D}_{12}$ is the diffusion coefficient of the solvent in the polymer, $\mathrm{D}_{\mathrm{p}}$ is the diffusion coefficient of the polymer in the solvent. The superscripts of the terms on the right hand side of equation 2.1 signify that solvent penetration and polymer swelling occur in opposing directions. The diffusion coefficient of the polymer, $D_{\mathrm{p}}$, can be related to the properties of the polymer backbone via the characteristic ratio, $C_{\infty}$, a dimensionless parameter which describes the effect of interdependent monomer-monomer bond rotations in the polymer backbone:

$$
C_{\infty}=\frac{\left\langle R_{g}\right\rangle^{2}}{n l^{2}}
$$

where $l$ is the length of a monomer unit, $n$ is the number of monomer units in the macromolecule, and $R_{g}$ is radius of gyration of the macromolecule. Upon rearrangement Equation 2.2 yields:

$$
\left\langle R_{g}\right\rangle=\left(n l^{2} C_{\infty}\right)^{1 / 2}
$$

The translational diffusion coefficient, $\mathrm{D}_{\mathrm{p}}$, of a macromolecule in solution is inversely related to the radius of gyration by:

$$
D_{p}=\frac{R T}{6 \pi \eta_{s} R_{g}}
$$


where $R$ is the gas constant, $\mathrm{T}$ is temperature, and $\eta_{s}$ is the viscosity of the solvent. The $C_{\infty}$ value for iPMMA is about $30 \%$ greater than that of PPMMA $^{53,54}$. Accordingly the diffusion coefficients of iPMMA are expected to be smaller than those of sPMMA, and the recess of the iPMMA front, $d S / d t$ (Equation 2.1), may be expected to be greater than that of SPMMA.

The effect of PMMA tacticity on the rate of dissolution is illustrated by the case of acetic acid. The rates of PMMA dissolution were compared by plotting the position of the polymer front versus time of exposure to solvent, see Figure 2.6. There is a period of time before dissolution of the sPMMA sample begins, and after commencement the velocity of the front appears to be less than that in iPMMA. The observation of an induction time has been observed in other amorphous polymer systems. In this instance the induction time may be related to the decreased diffusion of solvent through sPMMA compared to that in iPMMA, which would cause a longer period of solvent penetration prior to swelling and subsequent dissolution. The front velocity can be seen to differ between iPMMA and sPMMA by comparing the slope of the plots in Figure 2.6. The velocity of the polymer front apparently increases with increasing iso-content of the polymer backbone. The effect on velocity is either due to longer relaxation times for the sPMMA sample as compared to the iPMMA sample, or due to the larger diffusion coefficient of iPMMA compared sPMMA. Even though acetic acid would strongly promote dissolution through formation of polymer-solvent contacts (acid-carbonyl), the effects mentioned above cause a measurable difference in the dissolution behavior of the polymer films. 
Plots of solvent position in the iPMMA films versus time for these experiments showed an intermediate diffusion relationship between Fickian $\left(\mathrm{dS} \sim \mathrm{t}^{1 / 2}\right)$ and Case II ( $\mathrm{dS}$ $\sim$ t) kinetics, which is typical of entangled, glassy polymers. Plots of the polymer front location versus time show trends related to the nature of the solvent and tacticity of the polymer. Figure 2.7 shows that solvents with stronger Lewis acidic nature increased the dissolution rate of all the polymer samples: all systems with chloroform dissolved faster than those with acetic acid, and 2-ethoxyethanol only caused swelling in iPMMA. A shift in the carbonyl absorbance of solvated chains was observed in all systems containing chloroform, similar to the solvated chains the iPMMA/ 2-ethoxyethanol system. This shift could not be observed in the case of acetic acid due to broad, overlapping band for the carboxylic acid. Thus, it is suspected that polymer-solvent interactions drive the dissolution process in the cases of chloroform, acetic acid and 2ethoxyethanol. Isotactic PMMA dissolved faster in the benzene and toluene than in 2ethoxyethanol even though intermolecular interactions with the carbonyl group were not observed. Viscosity of the solvents, which is inversely related to solvent diffusion coefficients in Equation 1, provides further insight to the order of these observations. Despite development of intermolecular interactions, dissolution was slower in the more viscous solvent, 2-ethoxyethanol, than in the less viscous solvents, benzene and toluene. Possibly the most common approach for predicting compatibility for dissolution tests is application of Hansen solubility parameters ${ }^{46}$ (HSP). The argument for the applicability of HSPs goes that the free energy of mixing $\Delta \mathrm{G}_{\mathrm{m}}$ needs to be less than zero for dissolution to occur:

$$
\Delta \mathrm{H}_{\mathrm{m}}-\mathrm{T} \Delta \mathrm{S}_{\mathrm{m}}=\Delta \mathrm{G}_{\mathrm{m}}<0
$$


The enthalpic term $\left(\Delta \mathrm{H}_{\mathrm{m}}\right)$ approaches zero if the polymer and solvent have similar HSPs, and the mixing is promoted by entropy of mixing $\left(\Delta \mathrm{S}_{\mathrm{m}}\right)$. When applied to polymer dissolution, this approach omits the effect of chain disentanglement on dissolution. Table I contains the HSP values of the solvents and polymers screened in this experiment ${ }^{45}$. By comparison with Figure 2.6, which shows the movement of iPMMA in the various solvents with time, it can be seen that the rate of iPMMA dissolution does not necessarily follow trends predicted from the HSP approach. The dissolution of iPMMA was faster in toluene than 2-ethoxyethanol even though the solubility parameter of toluene is farther from that of PMMA.

\subsection{Conclusions}

The dissolution process of PMMA stereoisomers varied depending on backbone conformation and solvent choice. Isotactic PMMA, which has the lowest Tg, dissolved faster than other stereoisomers in all solvents. Chloroform and acetic acid, which may interact with the ester functionality, dissolved all PMMA stereoisomers. Through analysis of the carbonyl peak in dissolving systems, polymer-solvent interactions are suspected to drive the dissolution. The polymer-solvent interface was observed to recede uniformly for all systems containing isotactic PMMA, while eruption of polymer fragments was observed for syndiotactic and atactic PMMA in benzene and toluene. Kinematic models for polymer dissolution were shown to qualitatively address the differences in dissolution behavior via the polymer diffusion coefficient, which itself is a function of chain stereoregularity. 


\subsection{Works Cited}

1. J. Manijkow, J. S. Papanu, D. W. Hess, D. S. Soane, and A. T. Bell. Journal of the Electrochemical Society, 134, 2003 (1987).

2. J. L. Koenig. Advanced Materials, 14, 457 (2002).

3. O. Pekcan and S. Ugur. Polymer, 43, 1937 (2002).

4. W. J. Cooper, P. D. Krasicky and F. J. Rodriguez. Polymer, 26, 1069 (1985).

5. E. E. Parsonage, N. A. Peppas and P. I. Lee. Journal of Vacuum Science \& Technology B: microelectronics and nanometer structures, 5, 538 (1987).

6. K. Ueberreiter. "Diffusion in Polymers" in Diffusion in Polymers; J. Crank, Park G.S., Ed.; Academic Press: New York, 1968).

7. B. Narasimhan and S. K. Mallapragada. Recent Research Developments in Macromolecules Research, 3, 311 (1998).

8. E. Gipstein, A. C. Ouano, D. E. Johnson and O. U. I. Need. Polymer Engineering and Science, 17, 396 (1977).

9. A. C. Ouano and J. A. Carothers. Polymer Engineering and Science, 20, 160 (1980).

10. W. Wunderlich. "Physical Properties of Poly(methyl methacrylate)" in Polymer Handbook, 4th edition; E. H. I. J. Bandrup, E.A. Grulke, Ed.; John Wiley \& Sons, inc: New York, 1999) pp V87.

11. S. Doulut, P. Demont and C. Lacabanne. Macromolecules, 33, 3425 (2000).

12. K. E. Min and D. R. Paul. Journal of Polymer Science: Polymer Physics Edition, 26, 1021 (1988).

13. W. P. Hsu. Journal of Applied Polymer Science, 83, 1425 (2002). 
14. W. P. Hsu. Journal of Applied Polymer Science, 66, 1773 (1997).

15. W. P. Hsu. Journal of Applied Polymer Science, 86, 2720 (2002).

16. L. Hamon, Y. Grohens, A. Soldera and Y. Holl. Polymer, 42, 9697 (2001).

17. S. Talibuddin, L. Wu, J. Runt and J. S. Lin. Macromolecules, 29, 7527 (1996).

18. S. Radhakrishnan and P. D. Venkatachalapathy. Polymer, 37, 3749 (1996).

19. E. G. Crispim, I. T. A. Schuquel, A. F. Rubira and E. C. Muniz. Polymer, 41, 933 (2000).

20. W. B. C. Liau, C.F. Journal of Applied Polymer Science, 76, 1627 (2000).

21. M. Brogly, Y. Grohens, C. Labbe and J. Schultz. International Journal of Adhesion and Adhesives, 17, 257 (1997).

22. Y. Grohens, M. Brogly, C. Labbe and J. Schultz. European Physical Journal, 33, 691 (1997).

23. Y. Grohens, L. Hamon, G. Reiter, A. Soldera and Y. Holl. European Physical Journal E, 8, 217 (2002).

24. N. A. Peppas, J. C. Wu and E. D. von Meerwall. Macromolecules, 27, 5626 (1994).

25. H. R. Lee and Y. D. Lee. Chemical Engineering Science, 46, 1771 (1991).

26. B. Narasimhan and N. A. Peppas. Advances in Polymer Science, 129, 157 (1997).

27. I. Devotta, M. Badiger, P. R. Rajamohanan, S. Ganapathy and R. A. Mashelkar. Chemical Engineering Science, 50, 2557 (1995).

28. A. P. Safronov and L. V. Adamova. Polymer, 43, 2653 (2002).

29. B. K. Miller-Chou, J. L. Koenig. Progress in Polymer Science, 28, 1223 (2003). 
30. F. Rodriguez and P. D. Killian. Journal of Applied Polymer Science, 66, 2015 (1997).

31. D. F. Stamatialis, M. Sanopoulou and I. Raptis. Journal of Applied Polymer Science, 83, 2823 (2001).

32. O. Pekcan and S. Ugur. Journal of Applied Polymer Science, 74, 948 (1999).

33. S. Ugur and O. Pekcan. Polymer, 38, 5579 (1997).

34. S. Ugur and O. Pekcan. Polymer, 41, 1571 (2000).

35. P. Morrissey and D. Vesely. Polymer, 41, 1865 (2000).

36. J. Gonzalez-Benito and J. L. Koenig. Macromolecules, 35, 7361 (2002).

37. T. Ribar, J. L. Koenig and R. Bhargava. Macromolecules, 34, 8340 (2001).

38. T. Ribar, R. Bhargava and J. L. Koenig. Macromolecules, 33, 8842 (2000).

39. D. W. Rafferty and J. L. Koenig. Journal of Controlled Release, 83, 29 (2002).

40. B. K. Miller-Chou, J. L. Koenig. Macromolecules, 35, 440 (2002).

41. C. M. Snively and J. L. Koenig. Journal of Polymer Science: Part B, Polymer Physics Edition, 37, 2353 (1999).

42. C. M. Snively and J. L. Koenig. Journal of Polymer Science: Part B, Polymer Physics Edition, 37, 2261 (1998).

43. C. M. Snively and J. L. Koenig. Macromolecules, 31, 3753 (1998).

44. R. S. Challa, S. Q. Wang and J. L. Koenig. Applied Spectroscopy, 50, 1339 (1996).

45. E. A. Grulke. "Solubility Parameter Values" in Polymer Handbook (4th ed.; J. I. Bandrup, E.H.; Grulke, E.A., Ed.; John Wiley \& Sons, Inc: New York, 1999) pp VII 675. 
46. C. M. Hansen, Hansen solubility parameters: a user's handbook;(CRC Press:

Boca Raton, Fla., 2000).

47. L. Masaro and X. X. Zhu. Progress in Polymer Science, 24, 731 (1999).

48. B. G. Wang, T. Yamaguchi and S. I. Nakao. Polymer, 42, 5225 (2002).

49. D. Vesely. Polymer, 42, 4417 (2001).

50. B. G. Wang, T. Yamaguchi and S. I. Nakao. Journal of Polymer Science: Part B, Polymer Physics Edition, 38, 846 (2000).

51. T. Yamaguchi, B. G. Wang, E. Matsuda, S. Suzuki and S. I. Nakao. Journal of Polymer Science: Part B, Polymer Physics Edition, 41, 1393 (2003).

52. B. Narasimhan and N. A. Peppas. Journal of Polymer Science: Part B, Polymer Physics Edition, 34, 947 (1996).

53. M. Vacatello and P. J. Flory. Macromolecules, 19, 405 (1986).

54. R. Jenkins and R. S. Porter. Polymer, 23, 105 (1982). 


\begin{tabular}{|c|c|c|c|c|}
\hline Solvent & $\begin{array}{c}\text { Solubility Parameter } \\
\left(\mathrm{MPa}^{1 / 2}\right)\end{array}$ & $\begin{array}{c}\text { Distance from PMMA } \\
\left(\mathrm{MPa}^{1 / 2}\right)\end{array}$ & $\begin{array}{c}\text { Molar Volume }\left(\mathrm{cm}^{3 /}\right. \\
\mathrm{mol})\end{array}$ & H-bonding group \\
\hline Acetic Acid & 21.3 & 10.5 & 57.1 & Strong \\
\hline Chloroform & 19 & 7.8 & 80.7 & poor \\
\hline Benzene & 18.6 & 11.9 & 89.4 & poor \\
\hline 2-ethoxyethanol & 23.5 & 8.4 & 97.8 & medium \\
\hline Toluene & 18.2 & 10.7 & 106.8 & poor \\
\hline
\end{tabular}

Table 2.1. Characteristics of the solvents tested. Solubility parameter, molar volume and hydrogen bonding group from ref (45). Distance from PMMA calculated by: $\left[4\left(\delta_{\mathrm{d} 2}-\right.\right.$ $\left.\left.\delta_{\mathrm{d} 1}\right)^{2}+\left(\delta_{\mathrm{p} 2}-\delta_{\mathrm{p} 1}\right)^{2}+\left(\delta_{\mathrm{h} 2}-\delta_{\mathrm{h} 1}\right)^{2}\right]^{1 / 2} \operatorname{ref}(46)$. 


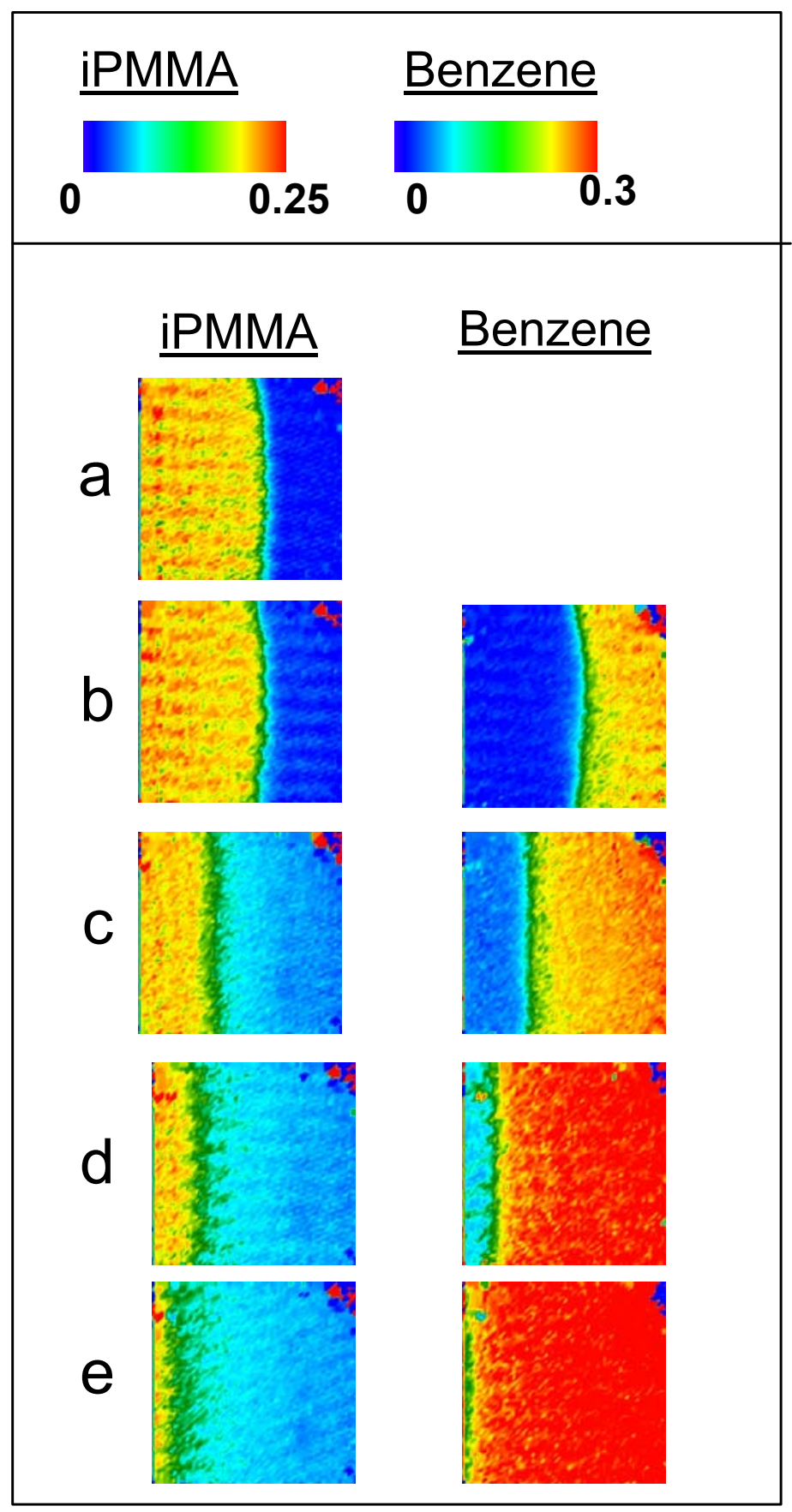

Figure 2.1. Dissolution of isotactic PMMA in benzene: a) before solvent addition; and after b) $2 \mathrm{~min}$, c) $6.6 \mathrm{~min}$, d) $11.25 \mathrm{~min}$, and e) 16 min of solvent exposure. iPMMA images plotted using the absorbance at $1755 \mathrm{~cm}^{-1}$; benzene images plotted using the absorbance at $1478 \mathrm{~cm}^{-1}$. 


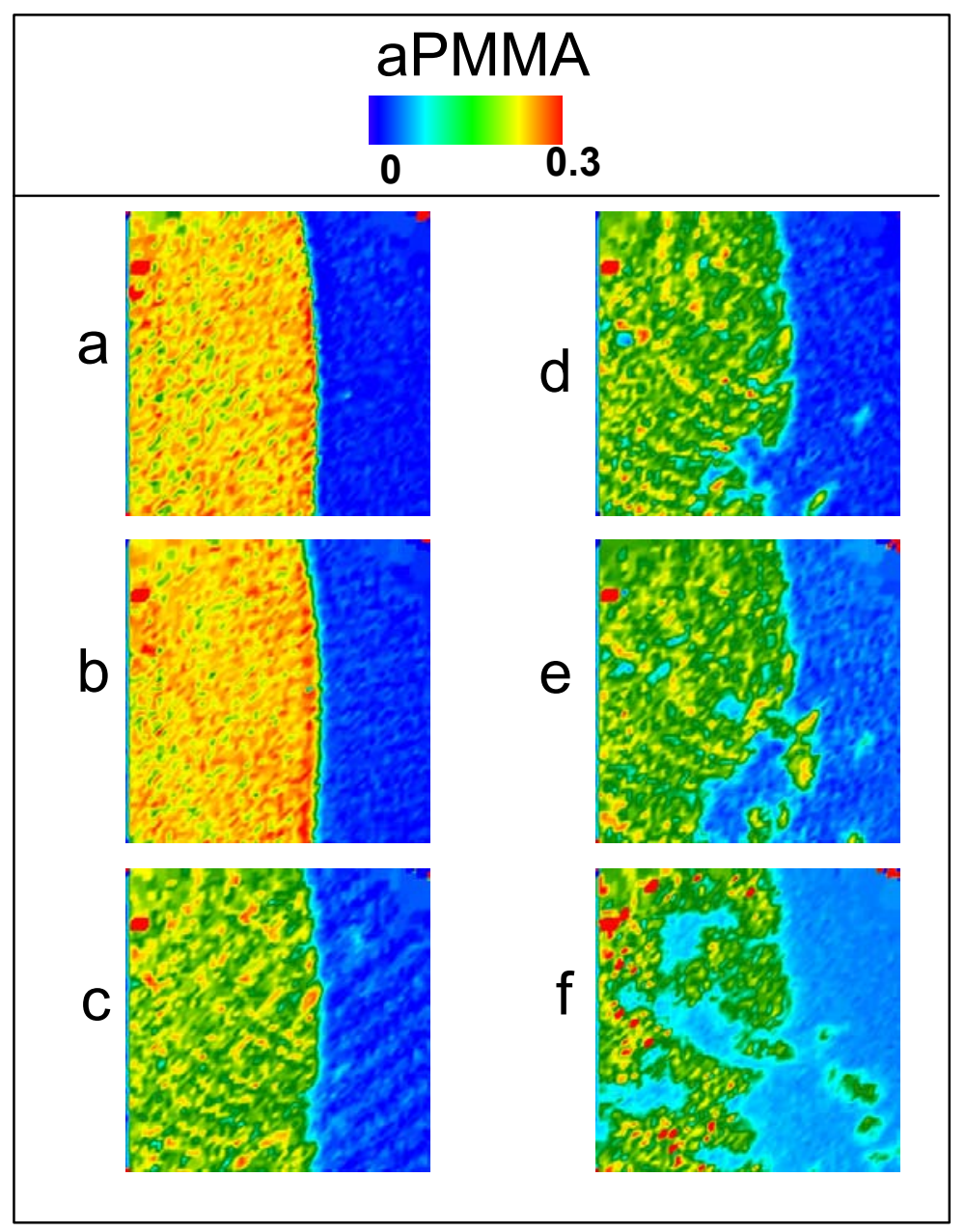

Figure 2.2. Eruption of aPMMA polymer fragments when exposed to benzene: a) before solvent addition; after b) 2 minutes; c) $35 \mathrm{~min}$; d) $46 \mathrm{~min}$; e) $51 \mathrm{~min}$; f) $57 \mathrm{~min}$ of solvent exposure. 


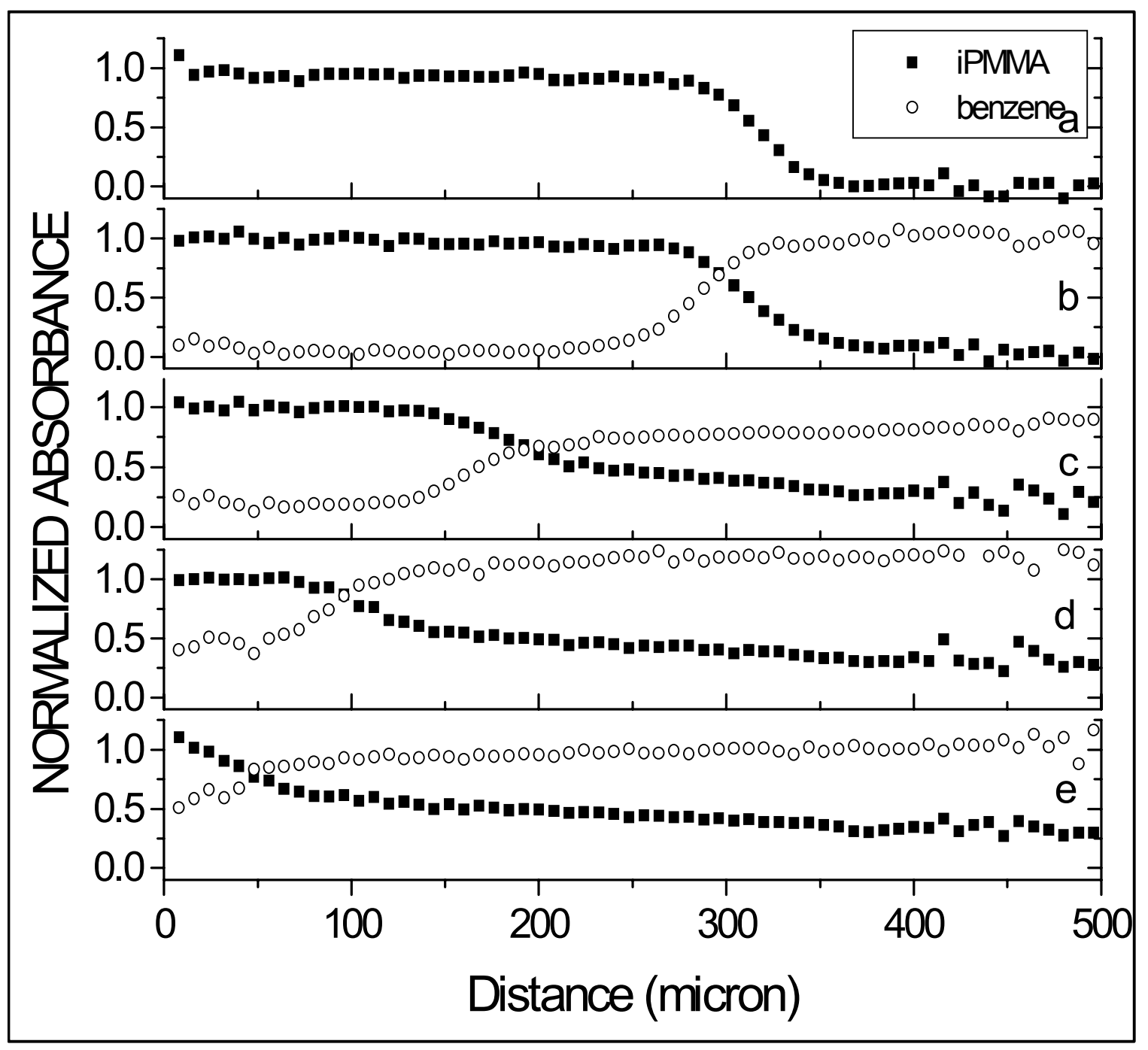

Figure 2.3. Normalized absorbance profiles of iPMMA dissolving in benzene: a) before solvent addition; and after b) $2 \mathrm{~min}$, c) $6.6 \mathrm{~min}$, d) $11.25 \mathrm{~min}$, and e) $16 \mathrm{~min}$ of solvent exposure. 


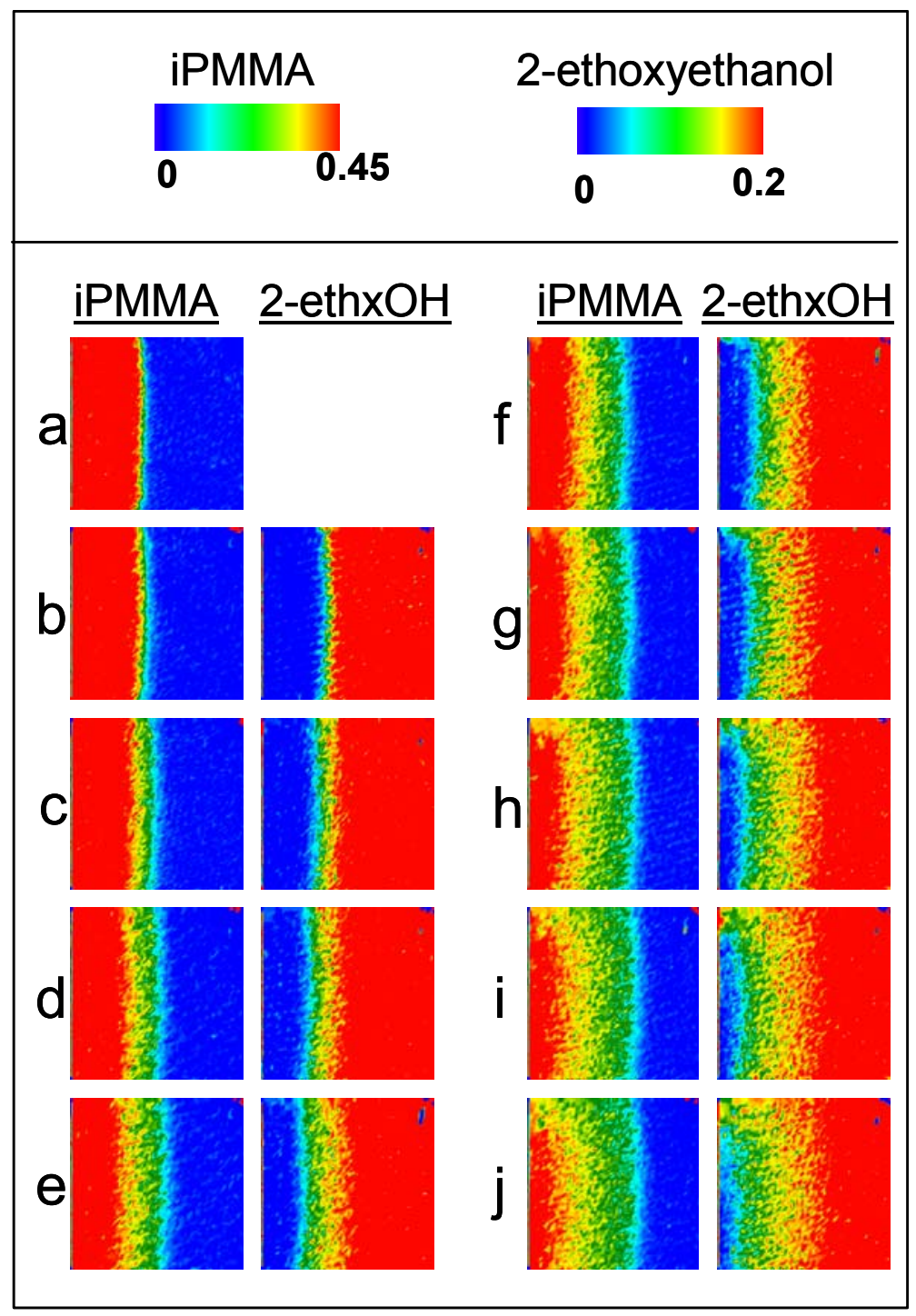

Figure 2.4. Images of iPMMA dissolving in 2-ethoxyethanol: a) before solvent addition; b) 2 minutes; c) $8 \mathrm{~min}$; d) $14 \mathrm{~min}$; e) $22 \mathrm{~min}$; f) $30 \mathrm{~min}$; g) $37 \mathrm{~min}$; h) $44 \mathrm{~min}$; i) $51 \mathrm{~min}$; j) $60 \mathrm{~min}$. Images generated by plotting the absorbance at $1750 \mathrm{~cm}^{-1}$ for iPMMA and $2857 \mathrm{~cm}^{-1}$ for 2-ethoxyethanol.. 


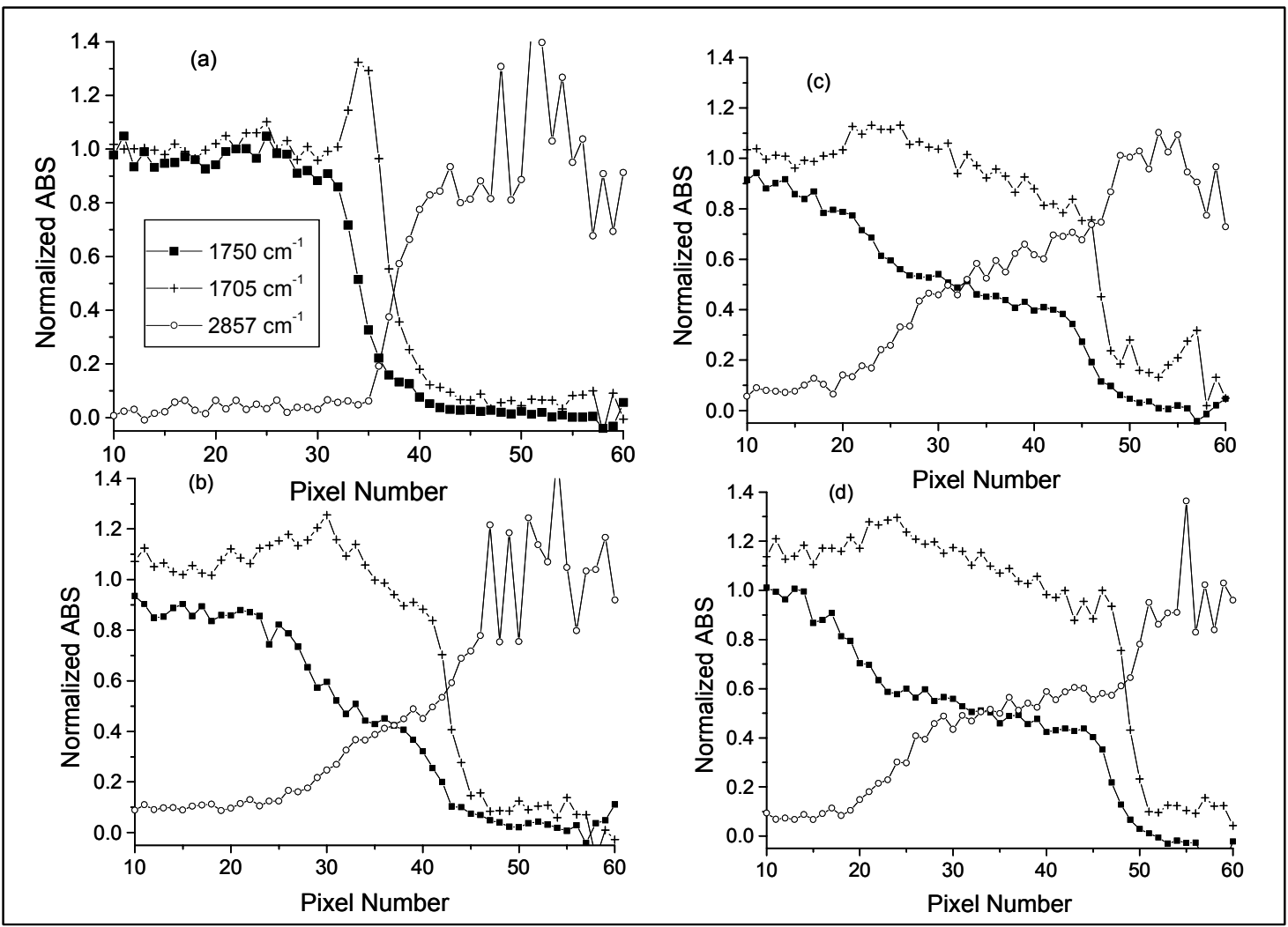

Figure 2.5. Normalized absorbance profiles for the iPMMA/2-ethoxyethanol system at: a) $2 \mathrm{~min}$, b) $25 \mathrm{~min}$, c) $44 \mathrm{~min}$, and d) $60 \mathrm{~min}$. Profiles shown for absorbances at 1750 $\mathrm{cm}^{-1}, 1705 \mathrm{~cm}^{-1}, 2857 \mathrm{~cm}^{-1}$. 


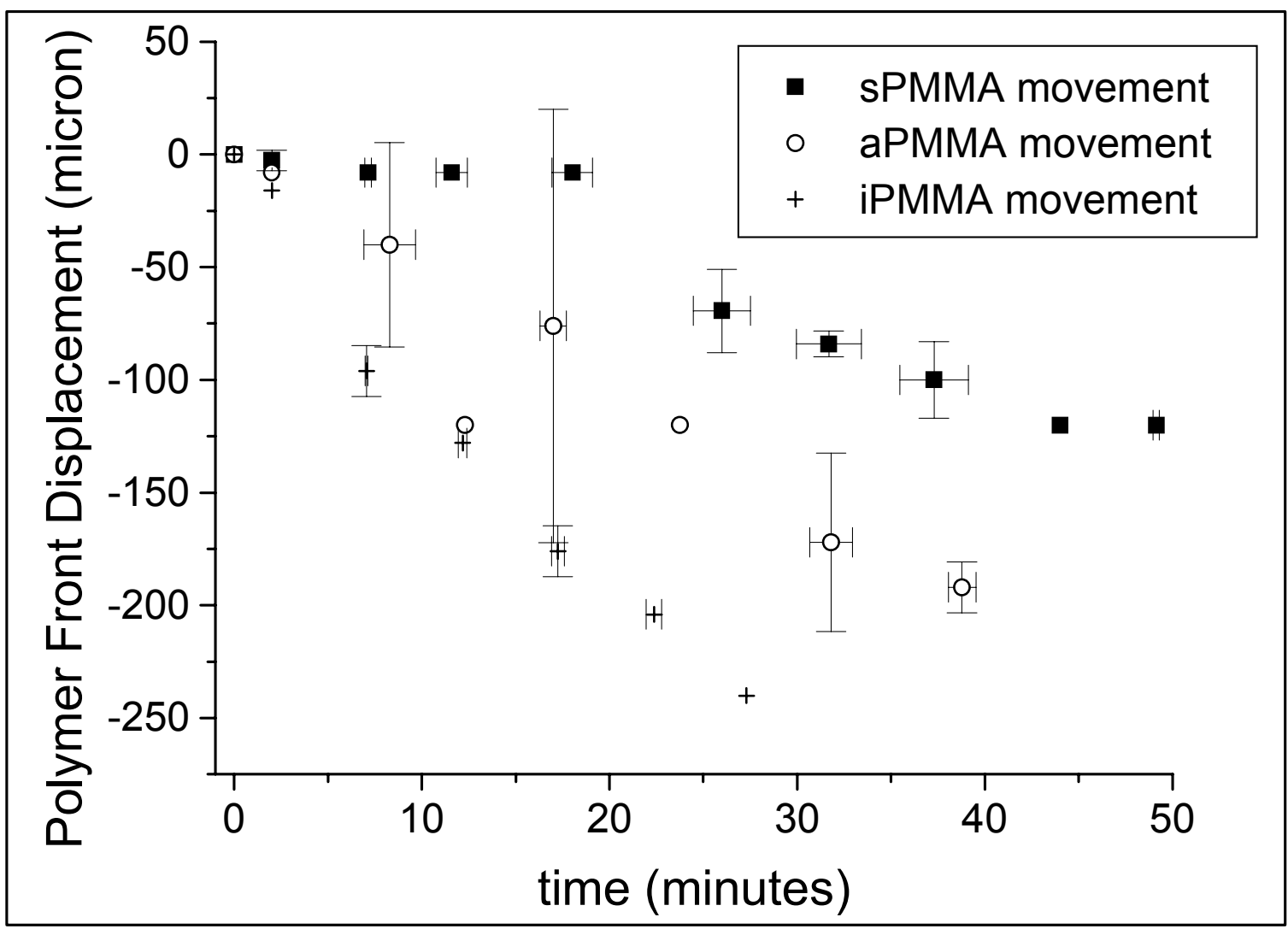

Figure 2.6. Displacement of polymer front versus time for three tacticities of PMMA films exposed to acetic acid. 


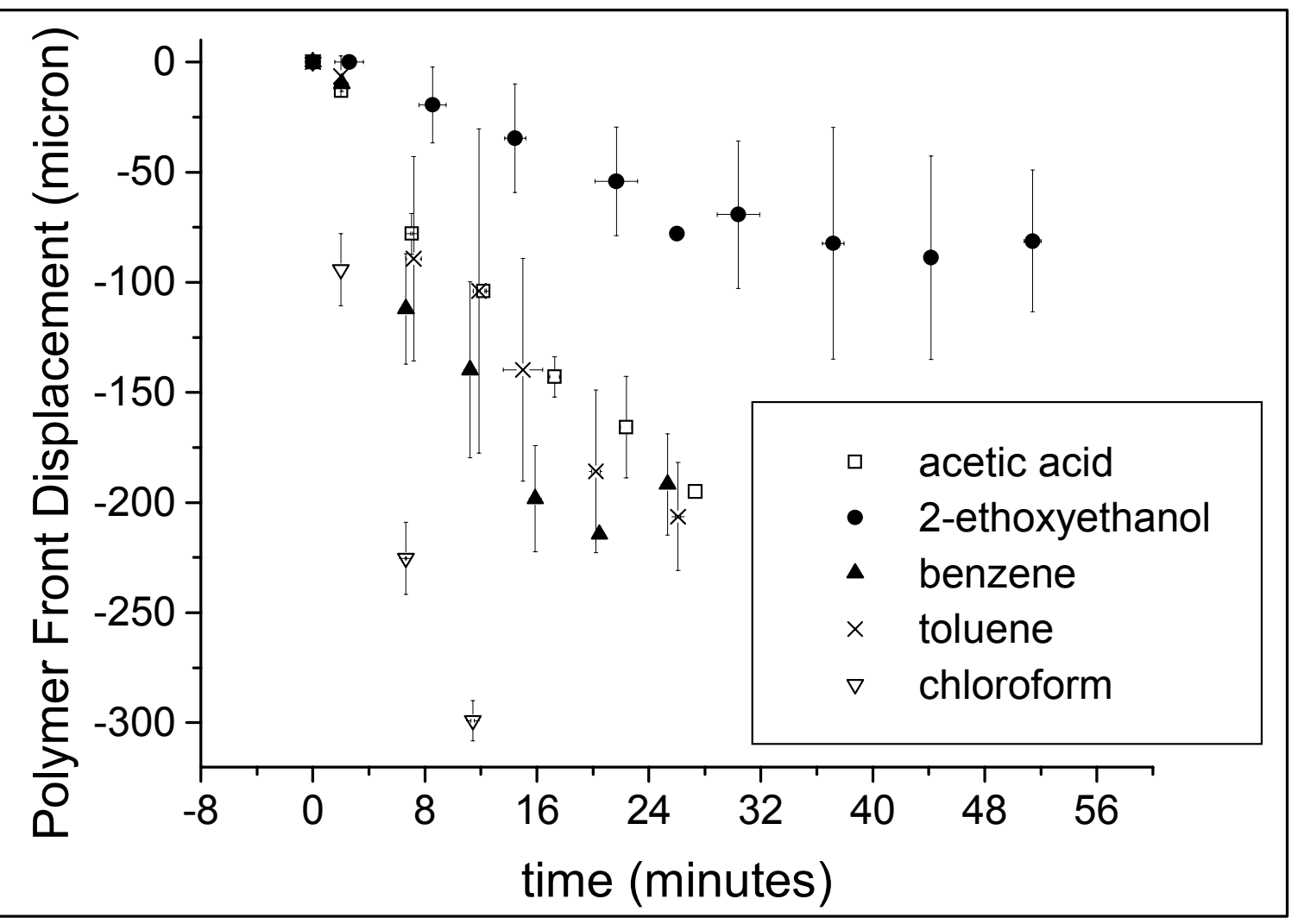

Figure 2.7. Displacement of the iPMMA front upon exposure to various solvents. 


\section{Regions of Interest in FTIR Imaging Applications: Diffusion of nicotine into Ethylene-co-Vinyl Acetate films}

\subsection{Abstract}

Effective image analysis of dynamic processes, such as diffusion and dissolution, requires precise reporting of component locations in space and time. An improved method for analyzing FTIR images is described which employs hypothesis testing in the spatial and temporal domains. Changes in the observed absorbance (over space and time) are revealed by comparison to a reference statistic, which can be tailored by choosing the size of a region of interest. This improved analysis method was used to compare the rates of diffusion of nicotine into poly (ethylene-co-vinyl acetate) film from aqueous solutions containing anionic and nonionic surfactants. Compared to a solution without surfactant, sodium dodecyl sulfate inhibited the uptake of nicotine from aqueous solution whereas Tween 40 enhanced the uptake. The nicotine diffusion rate also showed a dependence on the length of the hydrophobic segment of nonionic surfactants. These results demonstrate the roles of solubilization, wetting, and viscosity on diffusion-controlled drug release.

\subsection{Introduction}

Chemical imaging techniques are exceptional for characterizing chemical and morphological features of polymer systems. Fourier Transform Infrared (FTIR) imaging with focal plane array (FPA) detectors offers spectral, spatial and improved temporal resolution relative to microscopic mapping methods. FPA detectors have facilitated the

in situ study of dynamic processes such as diffusion and dissolution of polymer systems ${ }^{1-}$ 
6. Image quality and interpretation, however, can be compromised by low signal to noise ratios (SNR) particularly for species with weak absorbance bands. Accurate interpretation of FTIR images thus requires that true sample absorbances be distinguished from noise.

Model drug delivery systems have been studied using FTIR imaging ${ }^{3,7}$ but regions of interest in those systems have exhibited large absorbance changes over time. The primary analytical obstacle in FTIR imaging of diffusion processes is accurately locating the spatial position of trace absorbances. Under that restriction it has been difficult to differentiate between slow-diffusing systems. Recent drug delivery models have employed surfactants to control the release rate of drugs from matrices ${ }^{8-12}$, tablets ${ }^{13-}$ ${ }^{17}$, solutions ${ }^{18-21}$, and emulsions ${ }^{21-25}$. The ability of surfactants to slow or speed up diffusion of a hydrophobic drug from an aqueous solution have been demonstrated using dialysis followed by UV measurements ${ }^{20}$. Although surfactants significantly impact the uptake and/ or release of drugs, no in situ imaging study to date has demonstrated their effect. One complication of such a study is the detection of trace absorbance changes, which need to be distinguished from noise, within a region of interest.

A few methods have been suggested to improve SNR in FTIR imaging. The observed infrared signal in FTIR microscopy can be increased by utilizing a bright synchrotron radiation source ${ }^{26-30}$. The source acts as an intense point source of radiation, allowing an improvement of SNR by up to 1000 times, however such a source has not yet been coupled to an FPA. Pre-collection methods for image improvement include optimizing the rate of frame collection with spectrometer step rate ${ }^{31}$ and "pseudo- 
coaddition" of data sets ${ }^{32}$, however, both of these methods are time consuming for dynamic systems for which a sequence of images are collected for a single experiment.

Post-collection methods permit both selection and analysis of spatial regions of interest (ROIs) using the same data set. These methods reduce the time preceding collection of useful FTIR images. For instance, Budevska demonstrated multiple component analysis as a method for correcting the spectral data for non-linear optical effects that can cause baseline slope ${ }^{33}$.

Filtering interferograms and smoothing spectral data can eliminate high frequency signal fluctuations ${ }^{34}$. The method of coaddition of spectra from selected pixels in of the FPA has been shown to improve the spectral quality for regions with homogeneous composition $^{35}$. However the size requirement for the coadded pixel regions was simply based on the desired SNR.

Univariate analysis of the data cube from FTIR imaging experiments leads to the generation of false-color images that represent the spatial distribution of components. Dynamic processes such as diffusion and dissolution can be better visualized by two dimensional absorbance profiles. Absorbance profiles in FTIR images plot the intensity of a particular component (either peak height or integrated area) across one row of the FPA. The diffusion and dissolution work of this research group has analyzed essentially flat interfaces between polymers and diffusant/ solvent, thus adjacent rows of pixels ideally show identical absorbance profiles. However, for weak absorbance bands the profiles of adjacent rows can vary significantly. Due to the sample geometry employed, an average absorbance profile for a designated column of pixels can be calculated, thus smoothing the absorbance profiles ${ }^{32}$. For a particular column of pixels, all of which are 
expected to respond identically over time, the mean integrated absorbance and variance can be calculated from the population of 64 independent samples.

The intimate relationship between spatial and spectral information in FTIR imaging generates a criteria for selecting the number of pixels to co-add, i.e., selecting the size of the ROI. In general molecular absorption bands follow Beer's Law,

$$
\mathrm{A}=a b c
$$

The absorptivity $(a)$ and pathlength $(b)$ can be assumed to remain constant for a particular band, so that only changes in concentration (c) affect the measured absorbance (A). Within a region of detector elements the integrated intensity of a particular band exhibits a normal distribution, containing a mean and variance. The response for a particular region can be compared to those of a previous image by hypothesis testing on a regionby-region basis. The experimental test statistic for each ROI is defined as:

$$
\mathrm{t}_{\mathrm{o}}=\frac{\left(\overline{\mathrm{y}}_{1}-\overline{\mathrm{y}}_{2}\right)}{\sqrt{\left[\left(\mathrm{S}_{1}{ }^{2} / \mathrm{n}_{1}\right)+\left(\mathrm{S}_{2}{ }^{2} / \mathrm{n}_{2}\right)\right]}}
$$

In this instance, $\bar{y}_{2}$ is the mean integrated absorbance at a specific spectral range at a selected time and $\bar{y}_{1}$ is the absorbance at some later time; $S_{2}$ and $S_{1}$ are the variances of the integrated absorbance within the ROI at the times $\bar{y}_{2}$ and $\bar{y}_{1}$ are measured; $\mathrm{n}_{2}$ and $\mathrm{n}_{1}$ are the number of pixels in each ROI. Upon migration of an absorbing species across the spatial region of interest, the test statistic for some ROI will exceed the corresponding reference statistic, $\mathrm{t}_{\alpha, v}$; such a change indicates that the concentration within the ROI has changed from the previous value. The response of one column of pixels can be followed over time, as shown in Figure 3.1. For interpretation of the spatial distribution of a 
species, the reference statistic provides a threshold that imparts certainty to the report of a species' location. Two parameters can be chosen to impose a desired value of the reference statistic: size of the ROI (n) and the degree of certainty of the test $(\alpha)$. The size of the ROI is related to degrees of freedom, $v$, by Equation 2:

$$
v=\frac{\left(\mathrm{S}_{2}^{2} / \mathrm{n}_{2}\right)+\left(\mathrm{S}_{1}^{2} / \mathrm{n}_{1}\right)}{\left(\mathrm{S}_{2}^{2} / \mathrm{n}_{2}\right) /\left(\mathrm{n}_{2}-1\right)+\left(\mathrm{S}_{1}^{2} / \mathrm{n}_{1}\right) /\left(\mathrm{n}_{1}-1\right)}
$$

To illustrate the effectiveness of this analytical approach, a model diffusion system, which is meant to mimic a transdermal drug delivery device (TDD), will be considered. A similar system was studied by Rafferty and Koenig to demonstrate the effectiveness of FTIR imaging for monitoring nicotine diffusion into polymer films from solutions of various compositions ${ }^{3}$. EVA is used in a semi-permeable barrier for smoking cessation devices; nicotine permeates the polymer film from a reservoir and eventually contacts the skin. The previous work showed that the rate of nicotine diffusion depended on composition of the solvent reservoir: ethanol acted to swell the polymer film, enhancing the rate of nicotine uptake, while nicotine uptake from solutions without ethanol occurred significantly slower ${ }^{3}$. In this work surfactants were included in the reservoir, in the absence of ethanol, to demonstrate their effect on the nicotine uptake. The surfactant could either act to facilitate nicotine uptake by wetting the polymer surface, or it could inhibit uptake by stabilizing the nicotine in water solutions.

\subsection{Materials and Methods}

Poly (ethylene-co-vinyl acetate) ( $70 \%$ vinyl acetate), nicotine, and deuterium oxide $\left(\mathrm{D}_{2} \mathrm{O}\right)$ were obtained from SigmaAldrich. EVA samples were prepared by clamping a 
small amount of polymer between two calcium fluoride $\left(\mathrm{CaF}_{2}\right)$ slides then heating at $100^{\circ} \mathrm{C}$ for 60 minutes and $40^{\circ} \mathrm{C}$ for another 40 hours. Sample thickness was controlled by placing glass spacers, 15 micron in thickness, on the edges of the $\mathrm{CaF}_{2}$ plates, away from area that would be imaged. The samples were prepared by melting in order to obtain a thin uniform specimen without the use of solvents, which would have necessitated extensive drying of the sample. The polymer samples were kept in desiccators until immediately before use in order to prevent moisture absorption by the polymer. Nicotine solutions were prepared by mixing pure liquid nicotine with an aqueous $\left(\mathrm{D}_{2} \mathrm{O}\right)$ surfactant solution, resulting in solutions with $30 \%$ nicotine, $10 \%$ surfactant, $60 \%$ deuterium oxide by weight. The nicotine level in solution was chosen to be the minimum amount required to observe a characteristic absorbance, around $1620 \mathrm{~cm}^{-1}$, in the unprocessed spectral data. The diffusion experiments were performed at ambient laboratory conditions by the contact method $^{36}$, which delivers the solution to the polymer front through capillary action. Visual observations confirmed that nicotine solutions made contact with the polymer front immediately before data acquisition commenced. Each experimental run proceeded for approximately 40 minutes. The experimental setup crudely represents a TDD, merely consisting of a fluid reservoir and a semi-permeable polymer membrane. These experiments aim to qualitatively analyze the effect of surfactant on the initial stage of delivery from a TDD- diffusion of an active reservoir component into the polymer membrane.

All FTIR images were collected using a Digilab Stingray which consists of an FTS 6000 step-scan interferometer coupled to a UMA 500 microscope accessory with a 15x objective and a Santa Barbara focal plane array of $4096(64 \times 64)$ mercury-cadmium- 
telluride elements. Each image represents a $500 \times 500 \mu \mathrm{m}^{2}$ area and the spatial resolution, though ultimately limited by diffraction, is approximately $8 \mu \mathrm{m}^{2}$. A long pass filter was used to eliminate the effect of Fourier fold-over. A stepping rate of $5 \mathrm{~Hz}$ was used for the interferometer. A spectral resolution of $8 \mathrm{~cm}^{-1}$ was used and signal to noise ratio was increased by averaging 64 camera images per interferometer step. The temporal resolution of the experiments was limited by the duration of image acquisition, approximately 240 seconds, thus the time assigned to each data set was consistently taken to be the average time during an acquisition- 120 seconds after initialization. The resulting data cubes were processed using Win IR Pro 3.3. Absorbance profiles and statistical summaries for each region of interest were generated using Environment for Visualizing Images (ENVI) 4.0 and Microcal Origin 5.0.

\subsection{Results and Discussion}

For image analysis of dynamic processes like diffusion and dissolution, it is essential to precisely report the location of each component in an FTIR image. Figure 3.2 presents two ways of visualizing the uptake of nicotine by the polymer over time: Figure 3.2a show profiles for the absorbance change at particular locations profiles of the test statistic and Figure 3.2b shows the raw absorbance profiles for the integrated intensity of the nicotine-specific absorbance band between $1566 \mathrm{~cm}^{-1}$ and $1612 \mathrm{~cm}^{-1}$. As mentioned above, the benefit of the test statistic profile is the presence of a reference statistic for determining the threshold value (the dotted line in Figure 3.2a). The rate of nicotine uptake can be qualitatively measured by plotting the displacement of the nicotine diffusion front versus time; an accurate quantitative assessment of the diffusion process would require higher spatial and temporal resolution than this particular imaging 
apparatus can provide. The threshold level in Figure 3.2a is an objective value based on the reference statistic $\mathrm{t}_{0.05,30}=1.697$ : below the threshold it cannot be reported, with a degree of certainty 0.05 , that the integrated absorbance of the nicotine-specific signal exceeds that of the initial image. Figures $3.2 \mathrm{a}$ and $3.2 \mathrm{~b}$ includes arrows where the diffusion front is reported to reside at each time, the values in Figure $3.2 \mathrm{~b}$ are subjectively based on the profile shape.

Precise reporting of the nicotine front position has significant consequences when estimating the diffusion rate of nicotine. The diffusion process in this experiment is expected to exhibit Fickian behavior since the polymer membrane is above the glass transition temperature. Thus, a plot of the nicotine front position (or displacement) versus the square root of time is expected to be linear. Figure 3.3 shows such a plot for either method of reporting the nicotine position: objectively using the statistical profile (a), and subjectively based on the absorbance profile (b). Although the same data set was used to generate Figures 3.3a and 3.3b, the method of processing produces two interesting features. First, the x-intercept of these plots, which denotes an induction time for the diffusion process, apparently depends on the method used to generate the profile. Gravimetric nicotine uptake measurements, yet to be performed, would ultimately substantiate the presence of an induction time. Second, with the assumption that the xintercepts are accurate, the error explained by the Fickian diffusion model improves from 0.943 to 0.996 by using the statistical profile. The inherent consistency in reporting position from the statistical profile causes the improved fit.

While the discussion above demonstrates the application of hypothesis testing to detect trace absorbance changes across an ROI, other workers have discussed the 
determination limits for absorption spectroscopy. For an $\alpha$-value of 0.05 the amount signal required for quantitative analysis, i.e. the determination limit, should be 10 times greater that the noise level ${ }^{34}$ :

$$
\mathrm{A}_{\lambda}=10 \sigma_{\lambda}
$$

Although this signal level was utilized as a threshold in previous FTIR imaging diffusion work $^{32}$, it has not been applied for detecting spatial changes in a dynamic system. The SNR has also been shown to improve by co-addition of pixels ${ }^{35}$, which is synonymous with using larger n-values for the ROI in the procedures outlined above. The improvements of the SNR by coaddition are illustrated in Figures 3.4 and 3.5. The average spectra (Figure 3.4) were generated from ROIs with $\mathrm{n}=1,3,7,11$, and 19, then the means were centered by subtraction of the spectrum for $n=19$. Figure 3.5 shows the residual values of spectra after subtraction in the nicotine-specific absorption region, 1550-1650 $\mathrm{cm}^{-1}$. By visual inspection, the noise level decreases from 0.01 A.U. (one pixel) to approximately 0.002 for $n=11$. The determination limit in this case would decrease from 0.1 (A.U.) to 0.02 by co-addition of pixels. The location of the nicotine front was determined for the $\mathrm{n}=11$ sample by using the determination limit, 0.02 , as the threshold value. Plots of the nicotine displacement with time for both the proposed statistical approach and the determination limit approach are displayed in Figure 3.6. Qualitatively, the plots differ only slightly in slope but the resulting induction times could potentially be different: 13 minutes and 7 minutes via the statistical and determination limit approaches, respectively. A linear fitting to both approaches shows a slightly better fit for the statistical approach $\left(\mathrm{R}^{2}=0.958\right)$ compared to the determination limit approach $\left(\mathrm{R}^{2}=0.941\right)$ 
The initial stage of any permeation process involves adsorption of a diffusant to a substrate; thus the wetting ability of surfactants plays an important role in uptake measurements. The solutions examined in this work contained approximately 3 times more nicotine than surfactant by weight. Although the surfactant concentrations exceeded the critical micelle concentration (CMC), some free nicotine molecules could be assumed to occupy the aqueous phase. Thus, the effect of surfactant wetting at the polymer-solution interface could influence the uptake of nicotine. Surfactants are frequently used in drug delivery applications to control the solubility and activity of hydrophobic components. Khossravi investigated the kinetics of drug diffusion through a polymer films using low quantities of poorly water-soluble drugs in aqueous surfactant solutions $^{20}$. Drug diffusion from surfactant-containing solutions was slower than a drugwater mixture in all the systems tested in that work. Diffusion is most inhibited by strongly interacting surfactants above the CMC, whereas weakly interacting surfactants can have a smaller impact on diffusion rate. Although the formation of drug complexes can slow the diffusion process, the equilibrium rate between free drug molecules and drug complexes ultimately determines the amount of uncomplexed drug molecules that are able to permeate a membrane ${ }^{19}$.

Figure 3.7 demonstrates that the nature of the surfactant in the aqueous reservoir influences the uptake of nicotine by EVA. Compared to a solution without a surfactant, nicotine uptake was enhanced in the presence of $10 \%$ Tween 40 and inhibited by the presence of $10 \%$ SDS. In the case of SDS, the diffusion process could be inhibited either by surfactant complexation at the polymer interface, or by the formation of impermeable nicotine-SDS complexes in solution. For solutions containing Tween 40 the nicotine 
ingress appears to be sustained over time compared to solutions without surfactant, this result could be explained by improved wetting of the polymer-solvent interface by the surfactant in the absence of nicotine-Tween 40 complex formations. The effects of surfactant viscosity and wetting ability were investigated by measuring nicotine diffusion from solutions containing two grades of Tween surfactant - each grade has a characteristic ratio of hydrophilic and hydrophobic block segment lengths. Figure 3.8 shows the nicotine uptake behavior of solutions with $10 \mathrm{wt} \%$ of Tween 40 and Tween 80 for two independent trials, Trial 1 and Trial 2. The different uptake tendencies could be explained either by improved contact of nicotine with the EVA due to the wetting ability of the Tween 40 surfactant, or by the limited transport of the nicotine in the more viscous Tween 80 solution. Nokhouchi et al. compared the release rate of hydrophobic drug, propranolol, from hydroxypropylmethylcellulose-Eudragit matrices that incorporated either SDS or Tween species in the drug-containing matrix ${ }^{11}$. The inhibition or acceleration of drug release was rationalized by the ability of different surfactants to either solubilize the drug or change the local viscosity of the tablet interface. The higher viscosity surfactant in this work, Tween 80 , could slow diffusion from the bulk of the drug-containing reservoir, making nicotine less plentiful at the polymer-solution interface, resulting in slower nicotine uptake.

\subsection{Conclusions}

The signal-to-noise limitations of FTIR imaging have been addressed by a new method for analyzing polymer-liquid interfaces. The data from selected pixels was accumulated into a region of interest, whose characteristics permitted hypothesis testing on a point-by-point basis. Choosing the size of the region of interest has important 
consequences on the spectral noise level and subsequent analysis. Trace absorbance changes that occurred as nicotine permeated an EVA film were modeled with higher precision than previous techniques for absorbance profile analysis. The analysis new method was applied to uptake of nicotine, from solutions of containing different surfactants, by EVA films. The ability of different surfactants either to wet the hydrophobic polymer film or to form complexes with nicotine molecules either enhanced or inhibited the uptake of nicotine, respectively, relative to a solution without surfactant. Incorporation of nonionic surfactant, Tween 40 , caused faster nicotine uptake than a nicotine-water mixture, whereas that the same level of anionic surfactant, SDS, slowed the uptake. These observations can be attributed to complex formation and different wetting abilities of each surfactant on the polymer interface.

\subsection{Works Cited}

1. J. Gonzalez-Benito and J. L. Koenig. Macromolecules, 35, 7361 (2002).

2. B. A. Miller-Chou and J. L. Koenig. Macromolecules, 35, 440 (2002).

3. D. W. Rafferty and J. L. Koenig. Journal of Controlled Release, 83, 29 (2002).

4. T. Ribar, R. Bhargava and J. L. Koenig. Macromolecules, 33, 8842 (2000).

5. T. Ribar, J. L. Koenig and R. Bhargava. Macromolecules, 34, 8340 (2001).

6. J. P. Bobiak and J. L. Koenig. Applied Spectroscopy, 58, 1141 (2004).

7. C. A. Coutts-Lendon, N. A. Wright, E. V. Mieso and J. L. Koenig. Journal of Controlled Release, 93, 223 (2003).

8. S. K. El-Arini and H. Leuenberger. International Journal of Pharmaceuticals, 121, $141(1995)$. 
9. V. M. Rao, J. L. Haslam and J. S. Valentino. Journal of Pharmaceutical Sciences, 90, 807 (2001).

10. A. Nokhodchi, P. Khaseh, T. Ghafourian and M. R. Siahi-Shabad. S.T.P. Pharma Sciences, 9, 555 (1999).

11. A. Nokhodchi, S. Norouzi-Sani, M.-R. Siahi-Shadbad, F. Lotfipoor and M. Saeedi. European Journal of Pharmaceutics and Biopharmaceutics, 54, 349 (2002).

12. A. Kubis, M. Szczesniak and W. S. Musial. Ars Pharmaceutica, 41, 397 (2000).

13. V. P. Shah,J. J. Konecny, R. L. Everett, B. McCullough, A. C. Noorizadeh and J. P. Skelly. Pharmaceutical Research, 6, 612 (1989).

14. R. Bodmeier and O. Paeratakul. International Journal of Pharmaceutics, 70, 58 (1991).

15. K. Knop and K. Matthee. S.T.P. Pharma Sciences, 7, 507 (1997).

16. B. W. Barry and D. I. D. El Eini. Journal of Pharmacy and Pharmacology, 28, $210(1976)$.

17. S. Brown, G. Rowley and J. T. Pearson. International Journal of Pharmaceutics, 165, 227 (1998).

18. K. M. O'Driscoll and O. I. Corrigan. Drug Development and Industrial Pharmacy, 8, 547 (1982).

19. T. Loftsson, B. J. Olafsdottir and N. Bodor. European Journal of Pharmaceutics and Biopharmaceutics, 37, 30 (1991).

20. D. Khossravi. International Journal of Pharmaceutics, 155, 179 (1997). 
21. E. Khalil. Alexandria Journal of Pharmaceutical Sciences, 13, 5 (1999).

22. F. Pattarino, E. Marengo, M. R. Gasco and R. Carpignano. International Journal of Pharmaceutics, 91, 157 (1993).

23. P. Clément,C. Laugel and J.-P. Marty. Journal of Controlled Release, 66, 243 (2000).

24. O. Chambin, V. Be'rard, M. H. Rochat-Gonthier and Y. Pourcelot. International Journal of Pharmaceutics, 235, 169 (2002).

25. R. T. Lostritto and S. L. Silvestri. Journal of Parenteral Science and Technology, 41, 220 (1987).

26. P. Dumas, N. Jamin, J. L. Teillaud, L. M. Miller and B. Beccard. Faraday Discussions, 126, 289 (2003).

27. H. Y. N. Holman, M. C. Martin and W. R. McKinney. Journal of Biological Physics, 29, 275 (2003).

28. H.-Y. N. Holman, M. C. Martin and W. R. McKinney. Spectroscopy (Amsterdam, Netherlands), 17, 139 (2003).

29. P. Yu, J. J. McKinnon, C. R. Christensen, D. A. Christensen, N. S. Marinkovic and L. M. Miller. Journal of Agricultural and Food Chemistry, 51

30. P. Yu,J. J. McKinnon, C. R. Christensen, D. A. Christensen, N. S. Marinkovic and L. M. Miller. Journal of Agricultural and Food Chemistry, 51, 6062 (2003).

31. C. M. Snively and J. L. Koenig. Applied Spectroscopy, 53, 170 (1999).

32. R. Bhargava, T. Ribar and J. L. Koenig. Applied Spectroscopy, 53, 1313 (1999).

33. B. O. Budevska. Vibrational Spectroscopy, 24, 37 (2000). 
34. P. C. Meier and R. E. Zünd, Statistical methods in analytical chemistry;(John Wiley \& Sons: New York, 1993).

35. R. Bhargava, S.-Q. Wang and J. L. Koenig. Applied Spectroscopy, 54, 486 (2000).

36. R. S. Challa,S. Q. Wang and J. L. Koenig. Applied Spectroscopy, 50, 1339 (1996). 


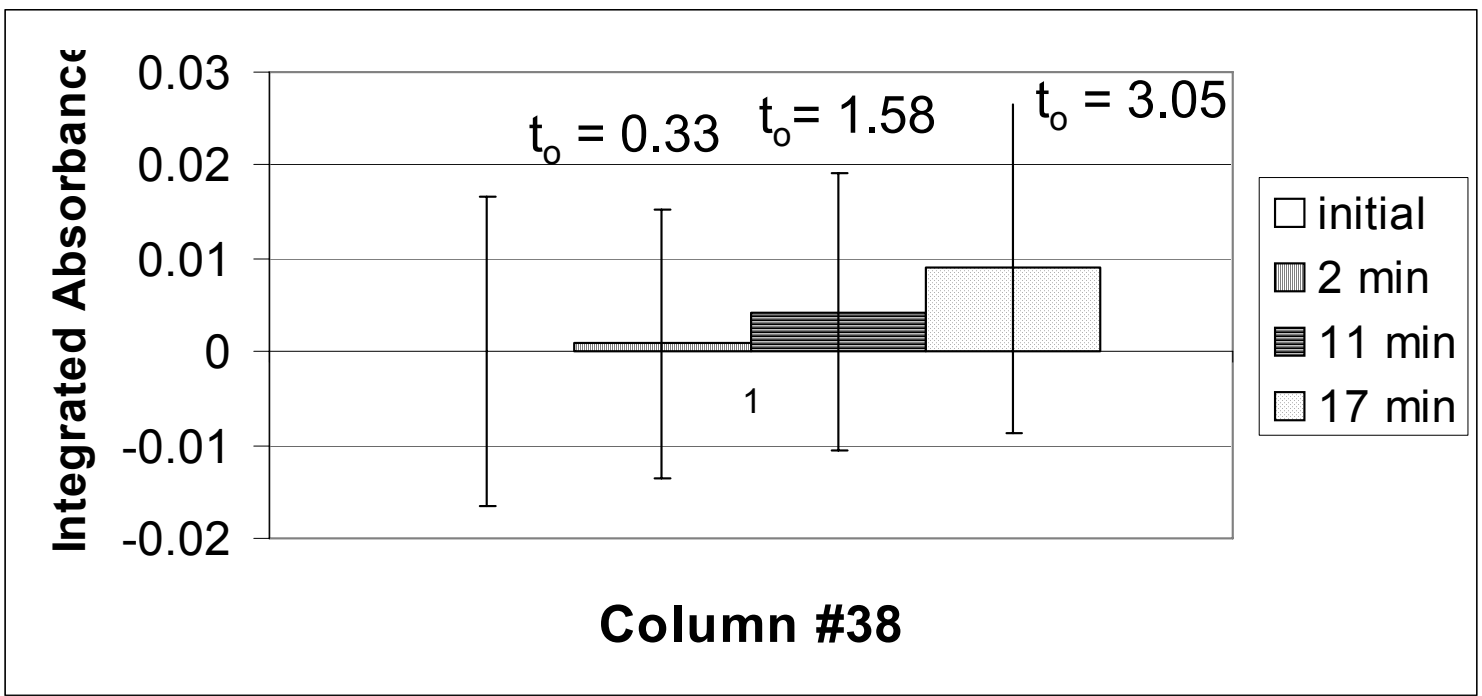

Figure 3.1. Mean integrated absorbance and deviation of a nicotine-specific band within one region of interest increases over time. Test statistic, $\mathrm{t}_{\mathrm{o}}$, is also noted for each time. The nicotine concentration within Column \#38 is concluded to have increased only after $t_{0}$ exceeds the reference statistic, $t_{0.05,30}=1.697$. The absorbance in Column \#38 increased after 17 minutes. 


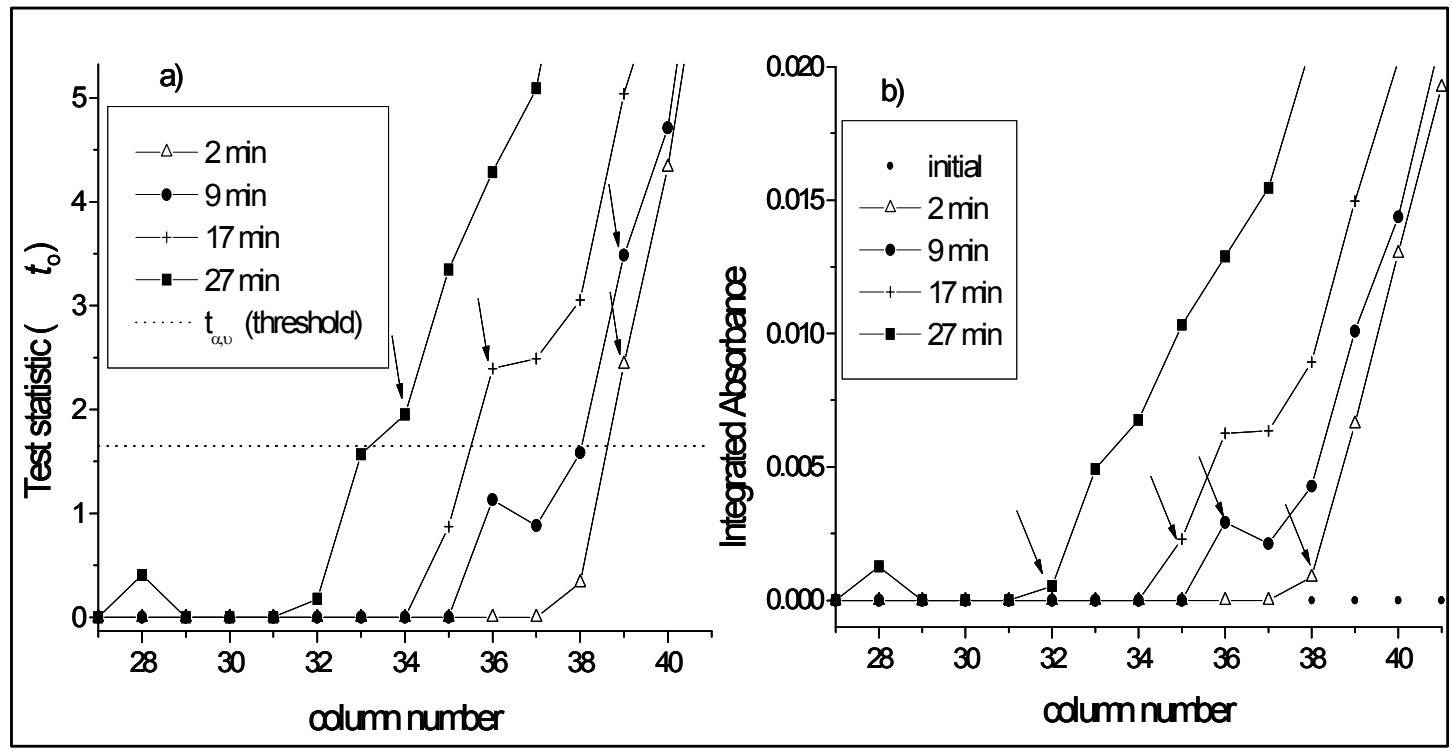

Figure 3.2. The characteristic nicotine absorbance near the diffusion front increases as diffusion proceeds over time. The process is represented by a) a statistical profile and b) a raw absorbance profile. The arrows in $2 \mathrm{a}$ and $2 \mathrm{~b}$ indicate the reported position of the nicotine diffusion front, based either on comparison the threshold value (2a) or subjectively based on the profile shape (2b). 


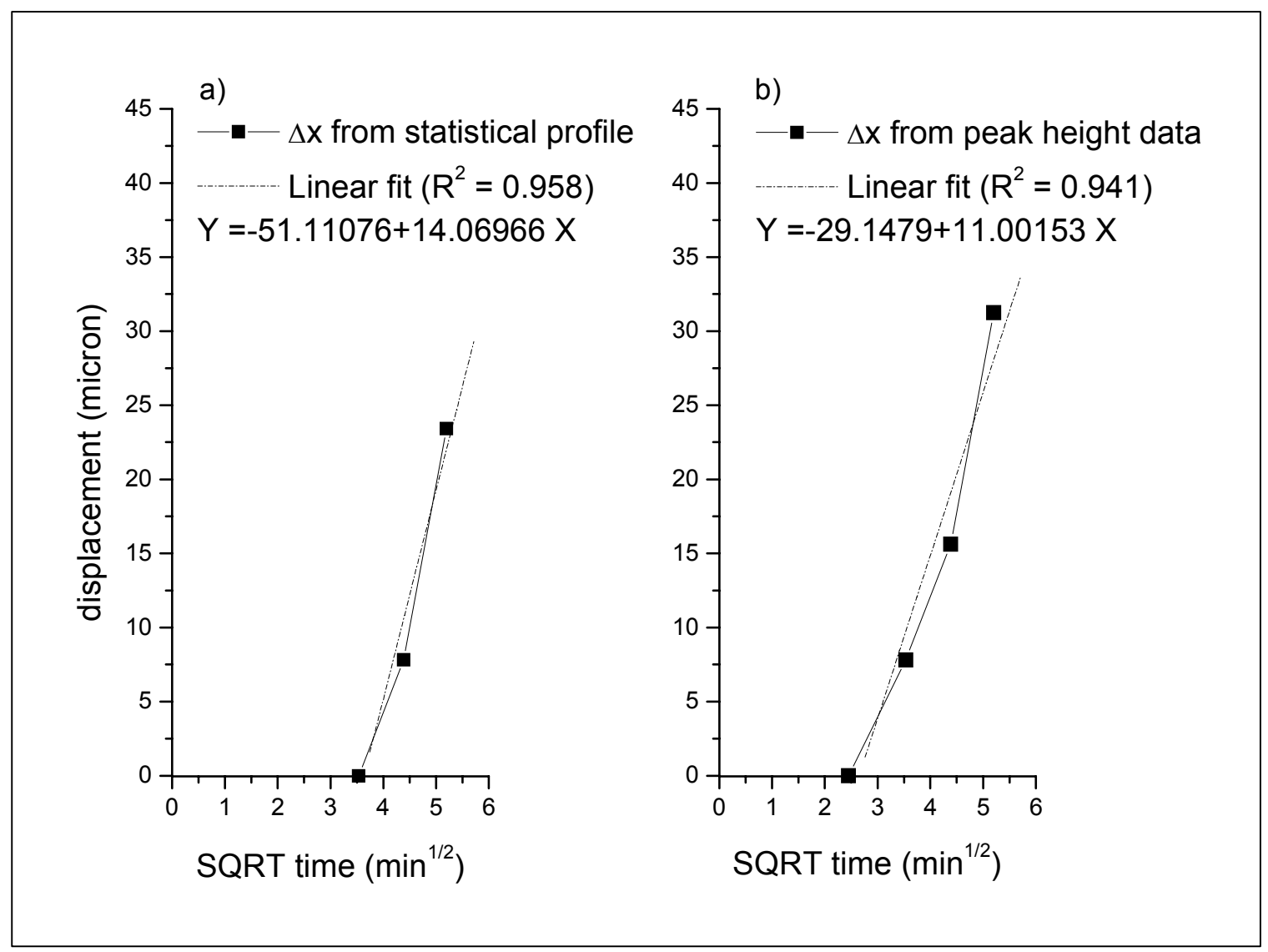

Figure 3.3. Progress of the nicotine diffusion front over time estimated from using the test statistic approach (a) and the raw absorbance profile (b). Linear fits are used since the diffusion process is expected to be Fickian. 


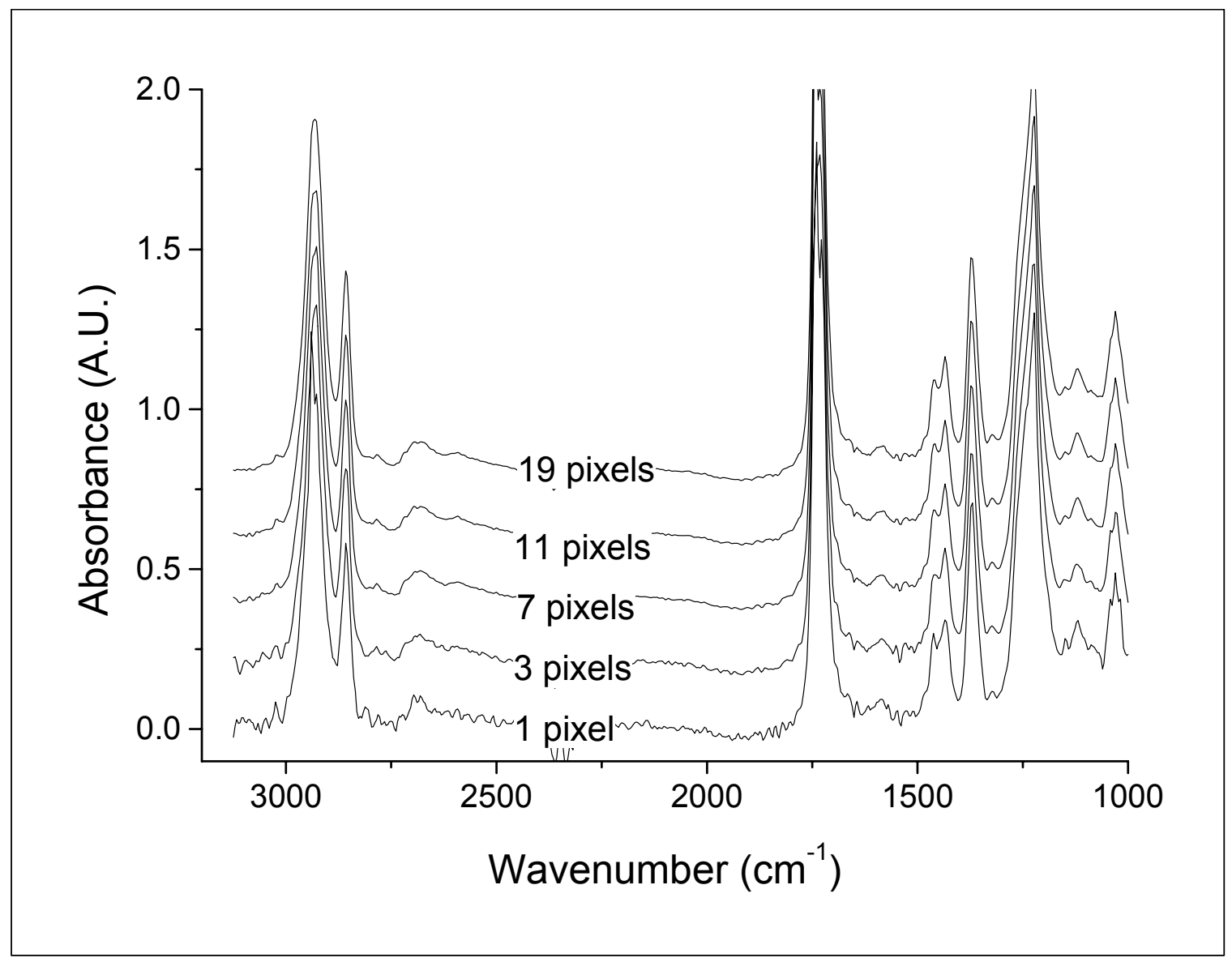

Figure 3.4. The average spectrum from a region of interest employing sizes, $n$, of: 1, 3, 7, 11, and 19 pixels. 


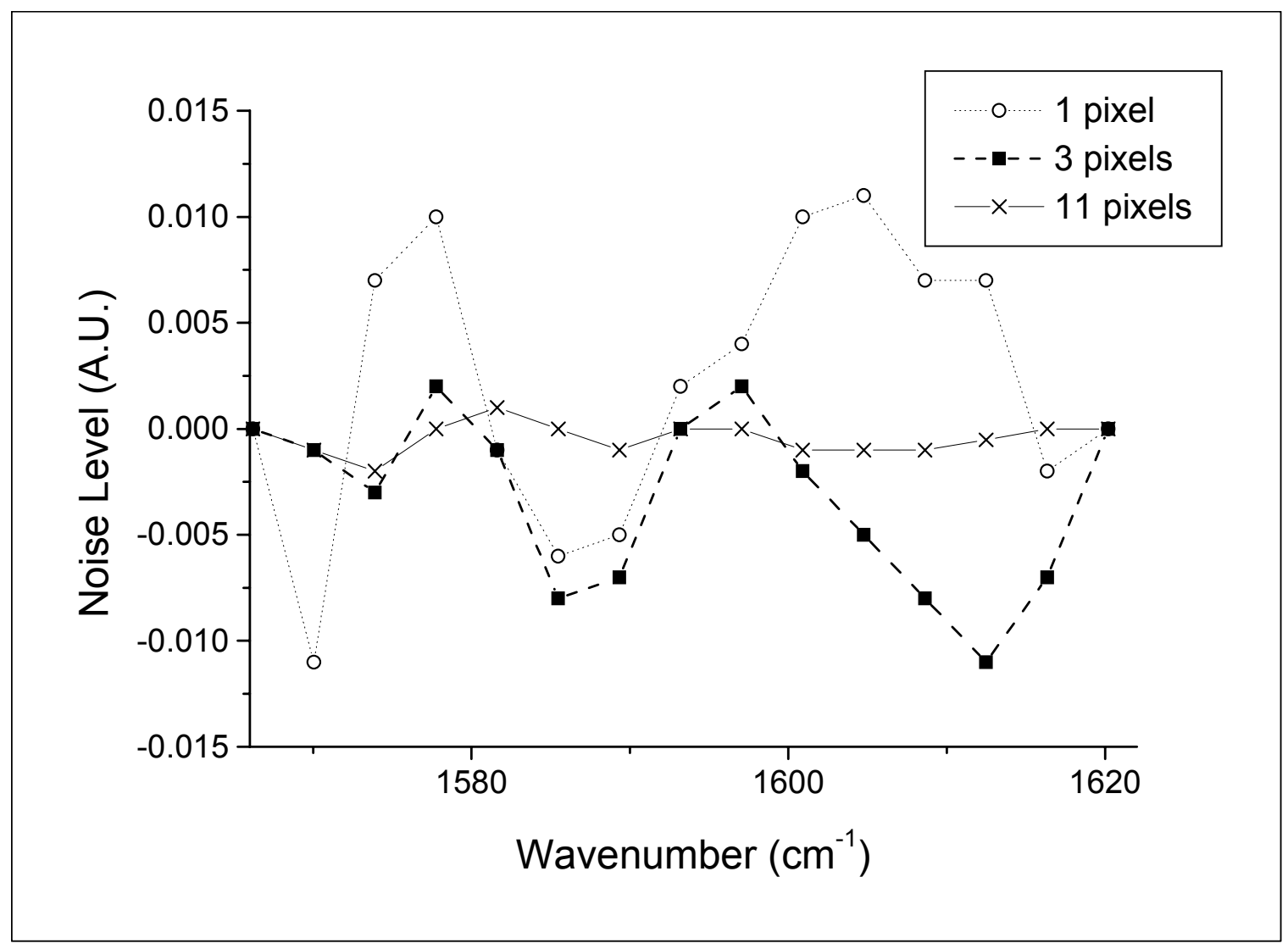

Figure 3.5. Mean centered spectra in the characteristic spectral region of nicotine. Plot indicates of the improved noise level as $\mathrm{n}$ increases. 


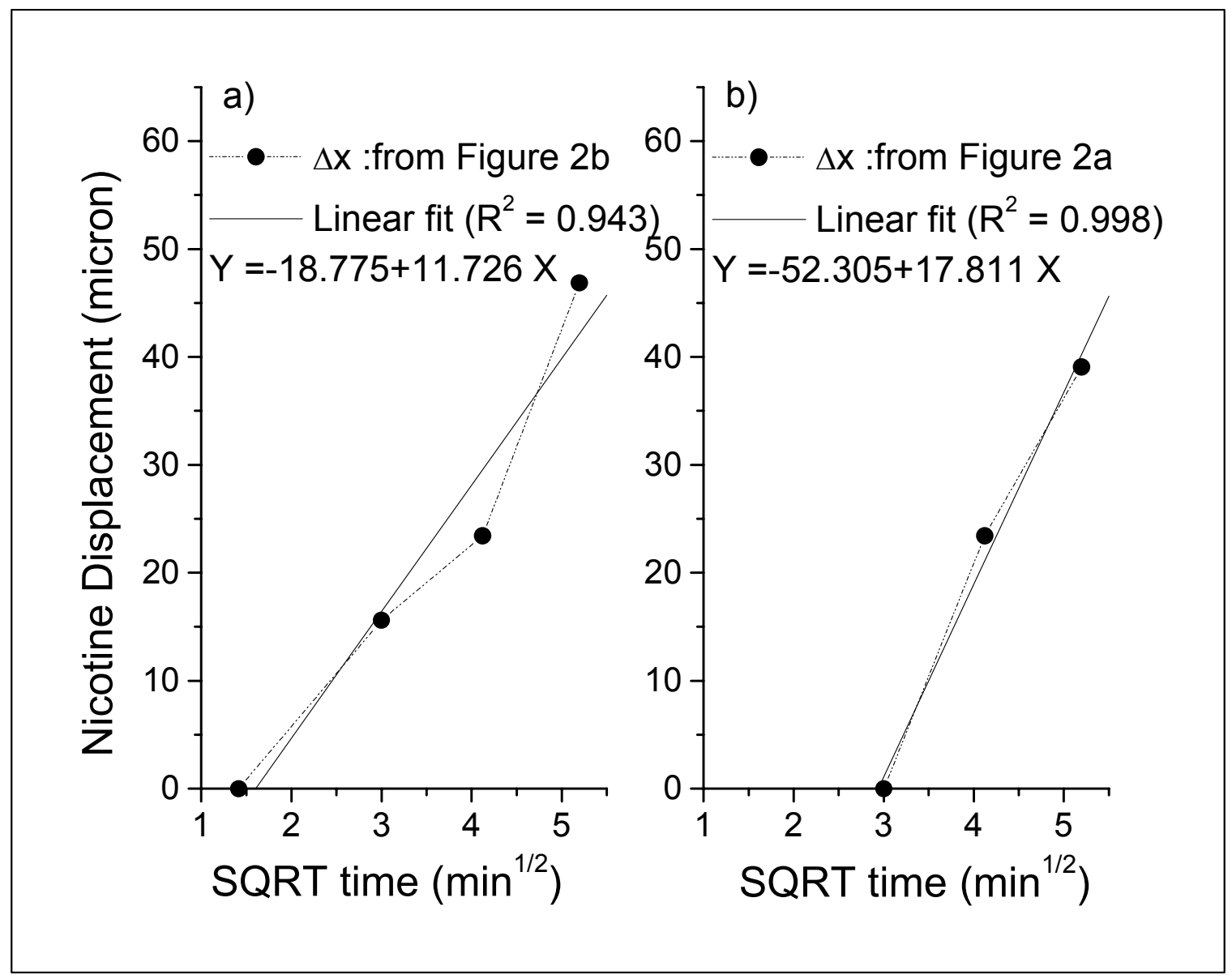

Figure 3.6. Comparison of diffusion kinetics as estimated by determination limit (a) and the test statistic approach (b). 


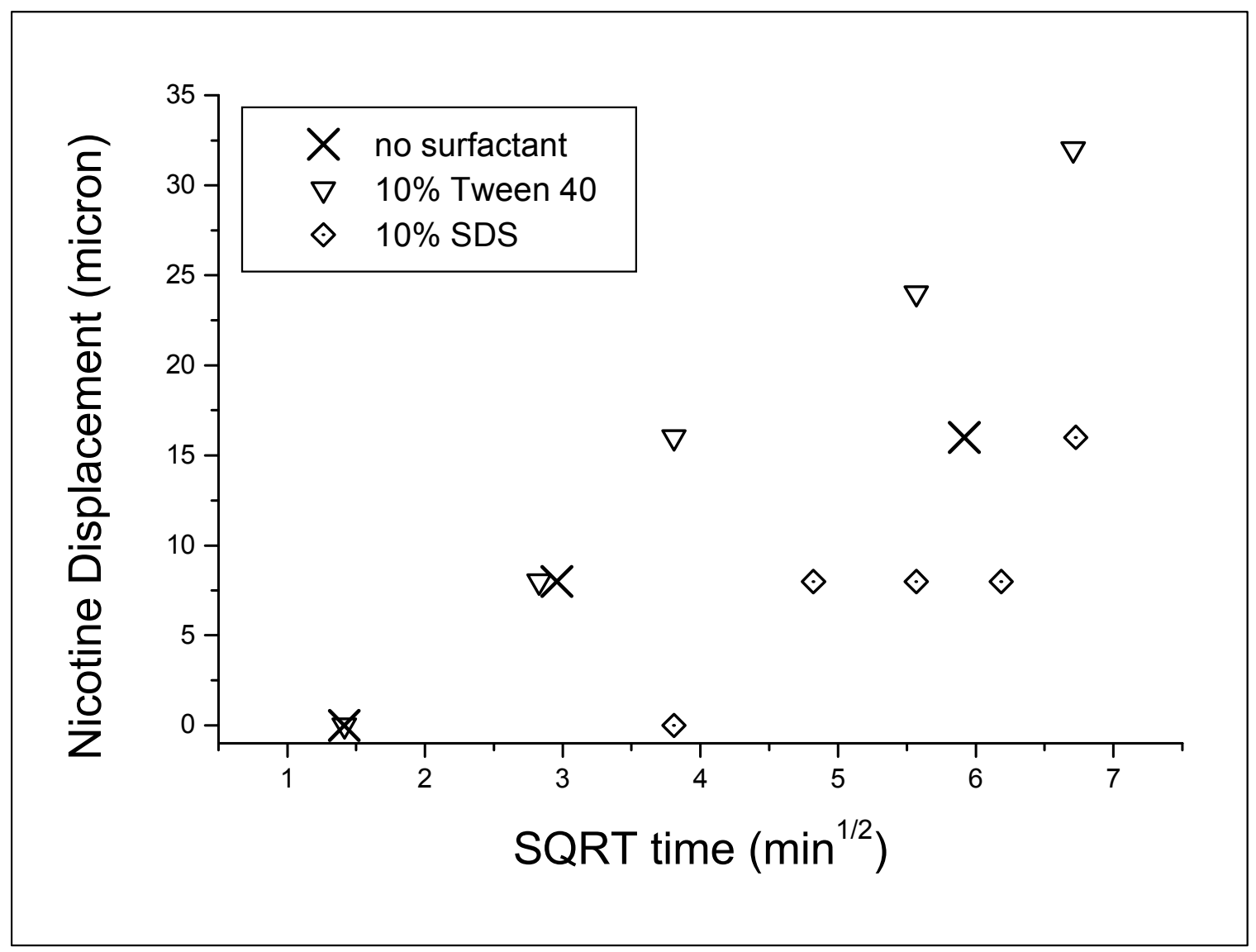

Figure 3.7. Nicotine permeation into EVA film from different solution compositions: no surfactant, non-ionic surfactant (Tween 40), anionic surfactant (SDS). 


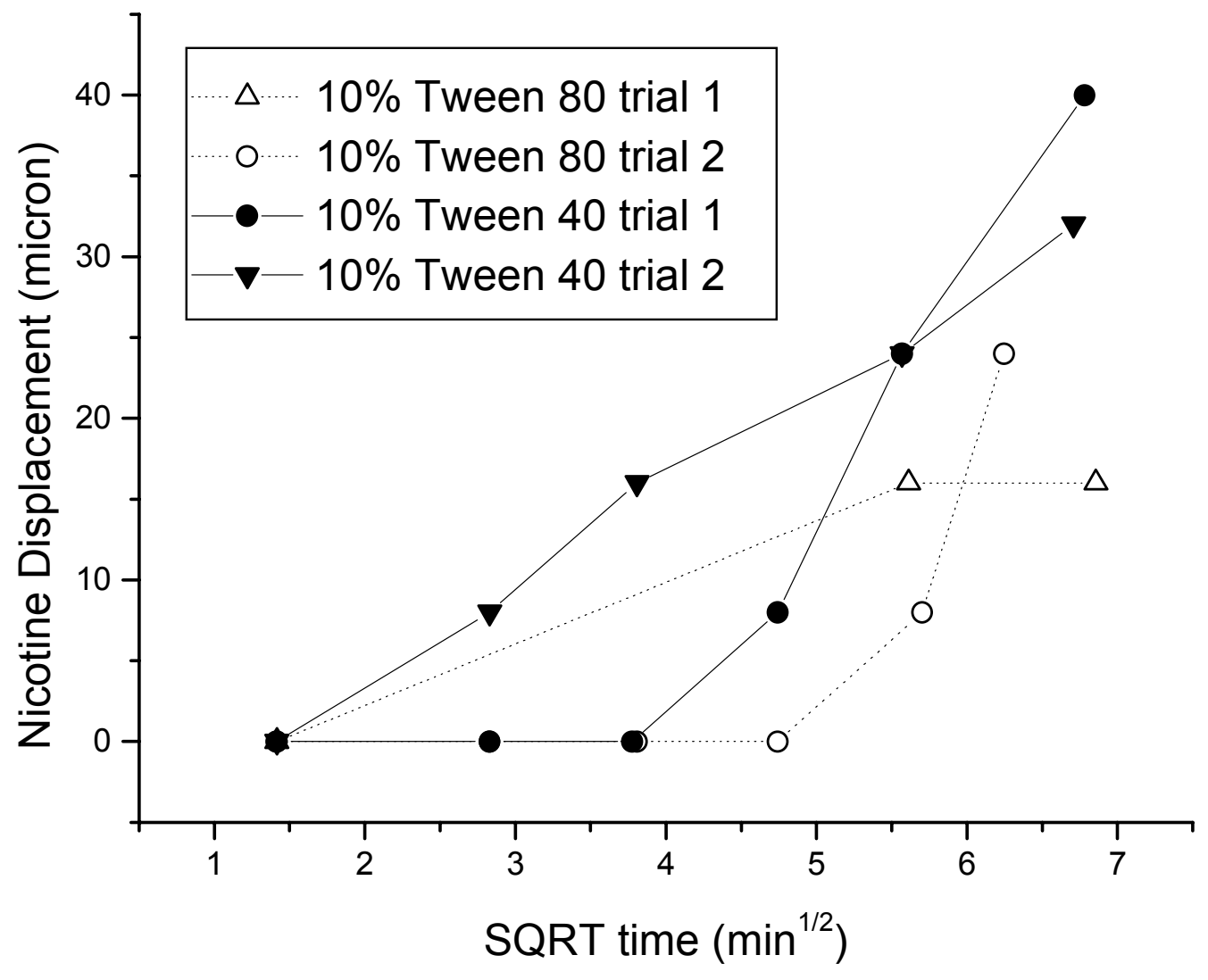

Figure 3.8. Displacement of the nicotine diffusion front (micron) over time $\left(\mathrm{min}^{1 / 2}\right)$ for two different surfactant identities, Tween 40 and Tween 80; either surfactant was present at $10 \mathrm{wt} \%$ in the solution. The results from two independent trials under the same conditions are shown to illustrate reproducibility. 


\section{Structural Classification of Dispersed Single Walled Carbon Nanotubes by Raman Imaging}

\subsection{Abstract}

Raman imaging was used to assess the distribution of isolated carbon nanotube features.

As a whole, the Raman spectra of the isolated tubes displayed a distribution of graphitic domain sizes. Photon scattering from carbon nanotubes is a resonantly enhanced process and since defects in the graphitic structure broaden the absorption bands, tubes with a higher number of defects produce weaker Raman signals. Two spectral imaging schemes were applied, Raman point mapping and Raman line imaging, to highlight the potential throughput advantage of line imaging. Spectral preprocessing of the line scanned data provided the same statistical distribution of tube features as observed as by point mapping, with the added benefit of faster image acquisition. The procedures applied here address the necessity to rapidly identify of distributions of individual tube structures from a bulk reaction product.

\subsection{Introduction}

Carbon nanotubes have potential in variety of applications including molecular-scale

electronic applications ${ }^{1,2}$, in which the properties of an individual tubes might influence the performance of a device. Single walled carbon nanotubes (SWCNTs) possess a one dimensional graphitic structure which exhibits either metallic or semiconducting tendencies. Bulk SWCNT samples often possess a mixture of semiconducting and metallic tubes with distributions of tube chiralities and diameters. Although several analytical protocols have been proposed to assess the character of bulk carbon nanotube 
samples ${ }^{3,4}$, there has been no fast method to analyze isolated tubes extracted from a bulk sample.

The bulk electronic properties of a SWCNT sample are produced by those properties of individual tubes in the material. An individual tube acquires its electronic properties during synthesis, when it assumes structural elements of chirality and diameter. Purification steps can remove chemical impurities from the reaction mixture but they do not homogenize tube chiralities, tube diameters, or the concentration of graphitic edges in a collection of tubes ${ }^{5,6,7}$. As applications of SWCNTs advance, it will be important to analytically assess the distribution of tube characteristics generated by a particular synthesis technique.

It has recently been shown that individual carbon nanotubes can be dispersed in surfactant solutions ${ }^{8}$. Upon casting of such a solution onto a substrate, Raman images display "hot spots" where the isolated tubes reside ${ }^{1,9,10}$. The Raman spectrum of carbon nanotubes strongly reflects the structural features of carbon nanotubes. Tube diameter and tube chirality control the tube's electronic band gap and, consequently, the extent of resonance enhancement at a particular excitation frequency. Defects in the graphitic structure, synonymous with graphitic domain boundaries, may also alter the electronic properties of SWCNTs. Graphitic domain boundaries have a characteristic Raman scattering band, often called the D band between 1260 and $1360 \mathrm{~cm}^{-1}$. The intensity of the $\mathrm{D}$ band $\left(\mathrm{I}_{\mathrm{D}}\right)$ has been empirically related to the size of the graphitic domain through comparison to the intensity of the $\mathrm{G}$ band $\left(\mathrm{I}_{\mathrm{G}}\right)$ which is generated by in-plane $\mathrm{C}-\mathrm{C}$ stretching vibration ${ }^{11}$. Thus, Raman imaging of solvent-cast tubes offers the opportunity 
to assess the distribution of graphitic domain sizes in a sample of dispersed carbon nanotubes.

\subsection{Materials and Methods}

Single walled carbon nanotube samples were obtained from Carbon Solutions, Inc. (Riverside, CA). They were prepared by electric arc method using Ni/Y catalyst and purified to contain low functionality and low amounts of catalyst. A trace amount of the nanotube material was sonicated for 30 minutes in a $1 \%$ SDS solution and centrifuged. A drop of the decanted solution was placed in the center of a tilted glass coverslip, allowing only a few of the dispersed tubes to adsorb to the surface before the solution ran off of the slide. The slides were dried under ambient conditions for 10 hours.

All Raman measurements were made with a Horiba Jobin Yvon LABRAM instrument outfitted with a $100 \mathrm{x}$ metallurgical objective $(\mathrm{NA}=0.9)$. The $632.8 \mathrm{~nm}$ line from a helium-neon laser (max operating power $17 \mathrm{~mW}$ ) was used as the illuminating source. The Rayleigh-scattered line was attenuated with a dielectric edge filter, the scattered light was dispersed by a 1800 grooves/cm grating, and then collected across a silicon-based CCD detector. The spectral resolution was approximately $1 \mathrm{~cm}^{-1}$.

The dispersed tubes within a 2500 square micron area were analyzed by both point mapping and line scanning acquisition schemes. For point mapping schemes, the incident light source was attenuated by a factor of 10 with a neutral optical density filter; the laser was not attenuated for the line scanning mode because scanning already compromised the power density. For point mapping schemes the microscope stage was rastered in 1 micron intervals in the $\mathrm{x}$ and $\mathrm{y}$ directions; the signal was two integrations of 
1 second were coadded. In line scanning mode the sample was moved in $1 \mu \mathrm{m}$ intervals in one direction; the signal for two integrations of 10 second were coadded to develop decent signal to noise. Even with longer acquisition times, the spectra from the line scanning scheme possessed large amounts of noise, which was reduced by two performing two types of preprocessing of the spectral information: median filtration or truncation of the inverse Fourier transform of the spectral data. All data acquisition and spectral processing was performed with LABSPEC software. All component-specific images and their relationships were analyzed using Microcal Origin 5.0.

\subsection{Results and Discussion}

Analysis of isolated tubes by point mapping

All graphitic carbon materials, including metallic and semiconducting carbon nanotubes, exhibit an in-plane C-C stretching vibration, often called the $\mathrm{G}$ band, between 1500 and $1620 \mathrm{~cm}^{-1}$. In order to visualize the presence of dispersed SWCNTs on a substrate, the intensity of the $\mathrm{G}$ band $\left(\mathrm{I}_{\mathrm{G}}\right)$ can be plotted for all positions in the sample plane, see Figure 4.1. For the most part, dispersed SWCNTs occupy only a few pixels in the entire image, these have been called "hot spots" in other work ${ }^{1,9,10}$. All of the SWCNTs are expected to be randomly distributed, and randomly oriented, across the sample plane. Different "hot spots" exhibit a different ratio between the $\mathrm{D}$ and $\mathrm{G}$ bands, $\mathrm{I}_{\mathrm{D}} / \mathrm{I}_{\mathrm{G}}$, which indicates that the SWCNTs in this sample are heterogeneous in terms of the graphitic domain size.

The first step in classifying the distribution of graphitic domain sizes is the isolation of only those areas where SWCNTs reside. Since all SWCNTs exhibit an intense $\mathrm{G}$ band, it is possible to apply an intensity threshold for that band over all pixels 
in the image. The intensity threshold needs to be above the level generated by shot noise to distinguish those pixels that contain SWCNTs from those pixels where tubes do not reside. Figure 4.2 displays histograms for the integrated intensities in two spectral regions of the raw data as well as for two forms of preprocessed data- the preprocessing steps were median filtration and truncation of the inverse Fourier transformed data. Figure 4.2a displays the number of pixels in the entire image that possess a particular range of integrated $\mathrm{G}$ band intensities values; Figure $4.2 \mathrm{~b}$ displays that for a spectral region containing no strong vibrations. Negative integrated intensity values are attributed to shot noise: random baseline fluctuations in regions where no intense bands are observed. For all pixels in the image, integration across the empty spectral region produces a normal distribution of integrated intensity values about zero, see Figure 4.2b. Both preprocessing methods that were applied to the raw data narrowed the distribution of integrated intensity values by synthetically smoothing the spectral baseline. Figures $4.2 \mathrm{a}$ and $4.2 \mathrm{~b}$ resemble one another because very few of the pixels in the image display an intense $\mathrm{G}$ band, but comparison of the expanded portions in Figure 4.2 reveals that a some pixels do exhibit high $\mathrm{G}$ band intensities. The $\mathrm{G}$ band intensity threshold values were determined by considering that the most negative intensity value generated by shot noise has approximately the same magnitude as the most positive intensity values from shot noise. Pixels containing $\mathrm{G}$ band intensities greater than the shot noise can safely be presumed to contain SWCNTs. The G band threshold was effectively decreased by preprocessing, allowing more of the dispersed material to be analyzed than was without preprocessing. The G band thresholds (Counts/ Sec) applied to the raw image, the median filtered image, and the IFT truncated image were 19, 13, and 7, respectively. 
The histograms in Figure 4.3 display the number of pixels in the image that contain particular ranges of $\mathrm{I}_{\mathrm{D}} / \mathrm{I}_{\mathrm{G}}$ values. The ratio between the $\mathrm{D}$ and $\mathrm{G}$ bands in any graphitic material is known to be inversely proportional to the size of the graphic domain

11. The graphitic domain size for a particular tube is acquired during tube synthesis, so an ideal nanotube synthesis procedure would produce a narrow distribution of graphitic domain sizes. When all pixels in the image are considered (solid markers in Figure 4.3), shot noise in the $\mathrm{D}$ and $\mathrm{G}$ band regions for empty pixels produces a distribution of values centered about zero which dominates the histogram. Upon isolation of only those pixels with intense $\mathrm{G}$ bands (open markers and expanded portion), it is apparent that a majority of the dispersed tubes have $\mathrm{I}_{\mathrm{D}} / \mathrm{I}_{\mathrm{G}}$ values below 0.5 . The expanded portion of the Figure 4.3 shows that tubes in the dispersed sample contain a distribution of graphitic domain sizes. Spectral preprocessing did not alter the characteristics of the $\mathrm{I}_{\mathrm{D}} / \mathrm{I}_{\mathrm{G}}$ distribution, but it did provide a higher pixel count, see Figure 4.4.

\section{Comparison between Point mapping and Line scanning schemes}

All of the results to this point have been based on images collected by a point mapping acquisition scheme. Line scanning schemes can decrease the image acquisition time by eliminating the need to raster the sample in one direction, the spectra from 100 adjacent points in the sample plane were collected in parallel. Line scanning schemes generally employ longer detector integration times since the intensity of the excitation source is distributed across one dimension of the sample plane. Even with a 20 -fold increase of detector integration time for line scanning, a complete Raman image could be acquired 5 times faster than for a point mapping acquisition scheme. Despite relatively long integration times, hyperspectral data from line scanning schemes still suffers from noise 
generated by the detector's integration process. The preprocessing steps introduced above (median filtration and IFT truncation) effectively correct for the noisy spectral data. Figure 4.5 displays histograms for the $\mathrm{I}_{\mathrm{D}} / \mathrm{I}_{\mathrm{G}}$ values in both point mapped and line scanned images, under different preprocessing methods. The IFT transformation apparently provided the best correlation between the point and line acquisition schemes.

\section{G-band Intensity}

Raman scattering from carbon nanotubes is a resonantly enhanced process. Figure 4.6 shows that the $\mathrm{G}$ band intensity increases with increasing graphitic domain size, i.e. decreasing $\mathrm{I}_{\mathrm{D}} / \mathrm{I}_{\mathrm{G}}$. This evidence suggests that tubes with large graphitic domains have larger Raman scattering cross sections, causing those tubes to scatter more intensely within a particular range of excitation frequencies. Data from the preprocessed, shown in Figures $4.6 \mathrm{~b}$ and $4.6 \mathrm{c}$, display this tendency more effectively since the impact of baseline fluctuations on the integrated band intensities is reduced.

Carbon nanotubes are essentially one-dimensional graphitic structures, and several features of their Raman spectra are common with graphite. The effect of domain size on the Raman spectrum of graphite has been investigated by irradiating graphitic samples with high energy particles. The concentration of defects in irradiated graphite can be controlled by limiting the duration or power of the irradiation source. Graphite irradiation with an argon ion beam, for instance, has been shown to cause both an increase in the $\mathrm{I}_{\mathrm{D}} / \mathrm{I}_{\mathrm{G}}$ value and a decrease in the integrated $\mathrm{G}$ band ${ }^{12}$. In another work, a series of proton-irradiated carbon nanotubes exhibited reduced ring breathing mode intensities, as well as broadened absorption spectra ${ }^{13}$, with increased dosage. The 
results shown in Figure 4.5 also support the notion that defects in a nanotube's graphitic structure disrupt the local electronic states and effectively reduce the extent of resonance enhancement.

\subsection{Conclusions}

Bulk carbon nanotube samples often contain a distribution of tube characters, both in terms of tube diameters and graphitic domain sizes. Raman imaging of dispersed carbon nanotubes offers a method to analyze individual tubes and assess the distribution of individual tube features in a bulk sample. Line scanning acquisition schemes can provide a 5-fold throughput advantage over point mapping schemes. Raman spectra of carbon nanotubes are resonantly enhanced and the $\mathrm{G}$ band intensity appeared to decrease with decreasing graphitic domain size. Defects in graphitic domains may disrupt the local electronic state of carbon nanotubes and ultimately reduce the extent of resonant enhancement for a particular tube. 


\subsection{Works Cited}

1. A. M. Rao, E. Richter, S. Bandow, B. Chase, P. C. Eklund, K. A. Williams, S.

Fang, K. R. Subbaswamy, M. Menon, A. Thess, R. E. Smalley, G. Dresselhaus and M. S. Dresselhaus. Science (Washington, D. C.), 275, 187 (1997).

2. $\quad$ S. Saito. Science (Washington, D. C.), 278, 77 (1997).

3. S. Arepalli, P. Nikolaev, O. Gorelik, V. G. Hadjiev, W. Holmes, B. Files and L. Yowell. Carbon, 42, 1783 (2004).

4. M. E. Itkis, D. E. Perea, R. Jung, S. Niyogi and R. C. Haddon. Journal of the American Chemical Society, 127, 3439 (2005).

5. $\quad$ N. E. Tran and S. G. Lambrakos. Nanotechnology, 16, 639 (2005).

6. A. C. Dillon, T. Gennett, K. M. Jones, J. L. Alleman and P. A. Parilla. Advanced Materials, 11, 1354 (1999).

7. Y. Zhang, Z. Shi, Z. Gu and S. Iijima. Carbon, 38, 2055 (2000).

8. M. J. O'Connell, S. M. Bachilo, C. B. Huffman, V. C. Moore, M. S. Strano, E. H. Haroz, K. L. Rialon, P. J. Boul, W. H. Noon, C. Kittrell, J. P. Ma, R. H. Hauge, R. B. Weisman and R. E. Smalley. Science, 297, 593 (2002).

9. V. G. Hadjiev, S. Arepalli, P. Nikolaev, S. Jandl and L. Yowell. Nanotechnology, $15,562(2004)$.

10. G. S. Duesberg, I. Loa, M. Burghard, K. Syassen and S. Roth. Physical Review Letters, 85, 5436 (2000).

11. F. Tuinstra and J. L. Koenig. Journal of Composite Materials, 4, 492 (1970). 
12. K. Nakamura and M. Kitajima. Physical Review B: Condensed Matter and Materials Physics, 45, 78 (1992).

13. P. P. Neupane, M. O. Manasreh, B. D. Weaver, R. P. Rafaelle and B. J. Landi. Applied Physics Letters, 86, 221908 (2005). 


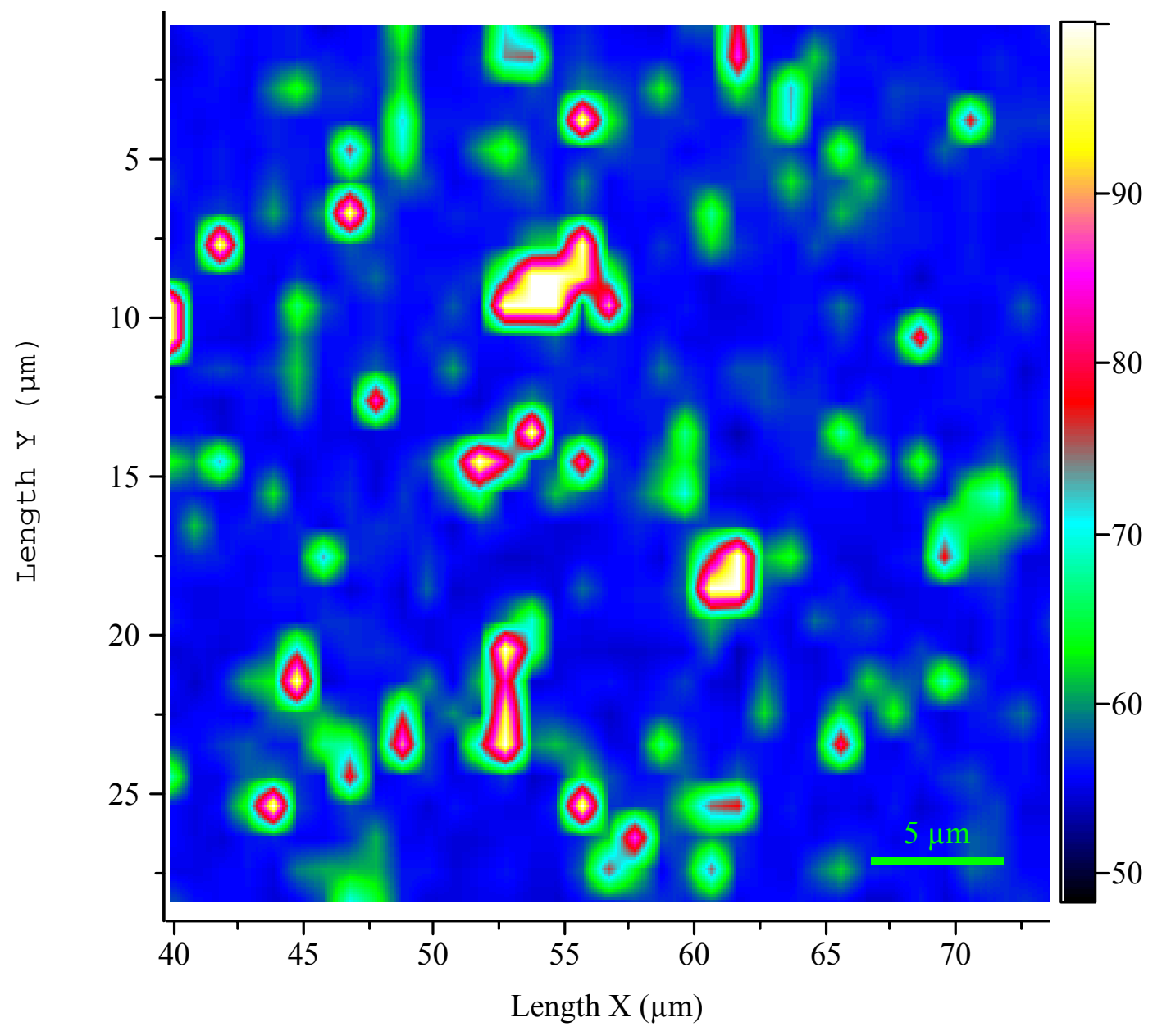

Figure 4.1. Image of dispersed single walled carbon nanotubes based on $\mathrm{G}$ band intensity $\left(1500-1620 \mathrm{~cm}^{-1}\right)$. 


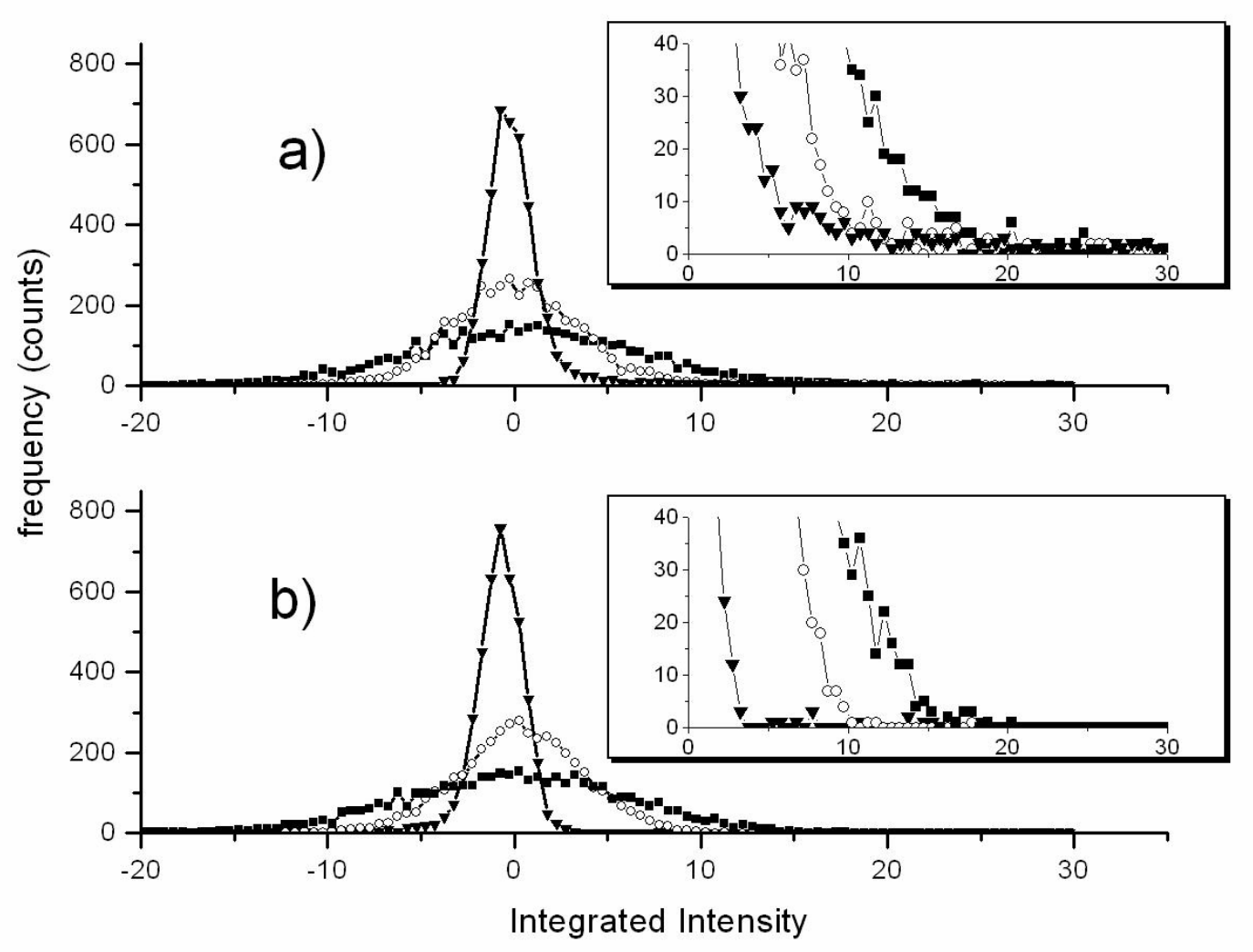

Figure 4.2. Histograms for the integrated intensity values in the spectral region of the $G$ band (a) and an empty spectral region (b). The raw spectral data ( $\mathbf{})$ was preprocessed by either median filtration (०) or IFT ( $\mathbf{\nabla})$. 


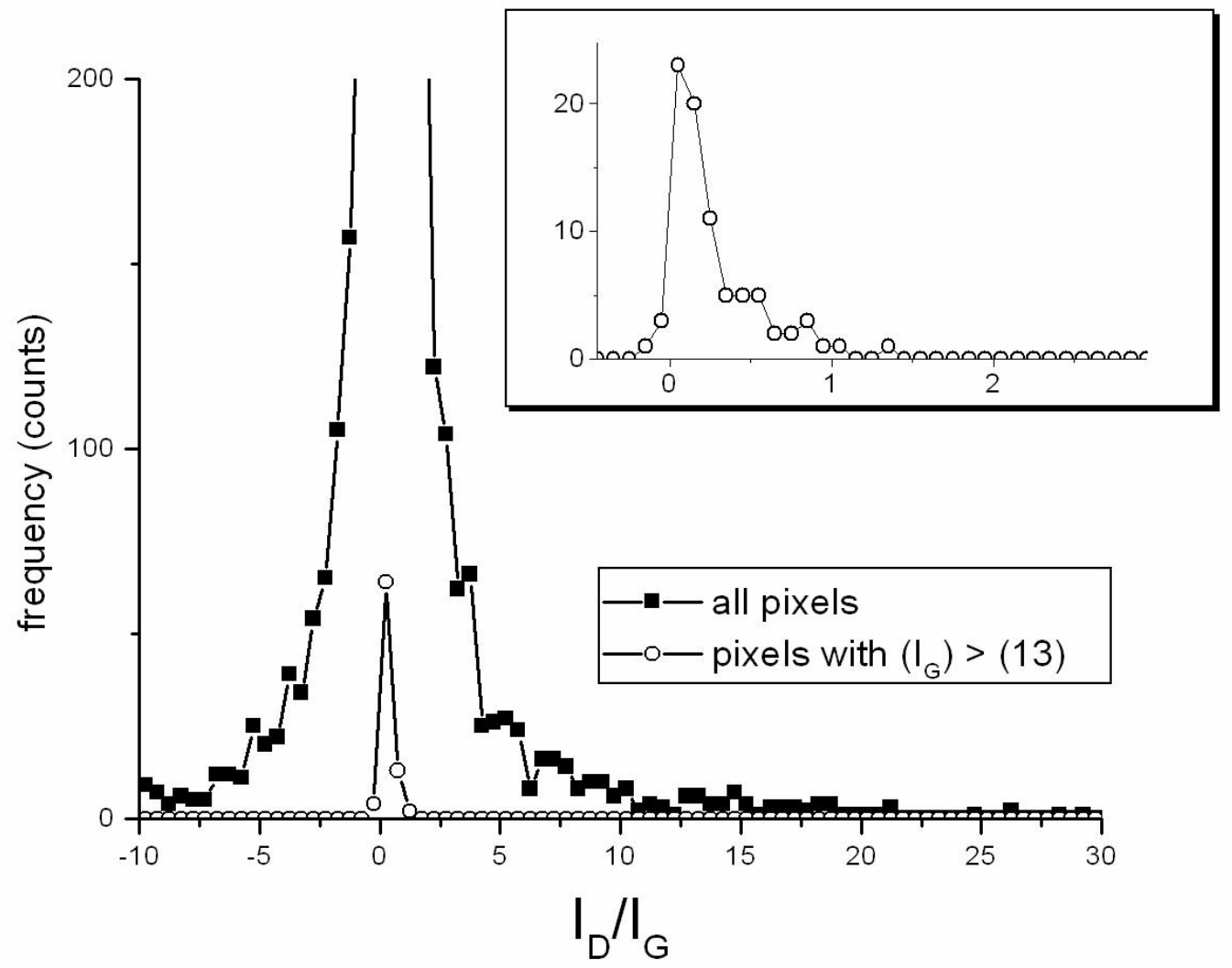

Figure 4.3. Histogram of the $\mathrm{I}_{\mathrm{D}} / \mathrm{I}_{\mathrm{G}}$ value for upon median filtration of the raw spectral data, displayed for all pixels in the image ( $\mathbf{-}$ ) and for only those pixels with G-band intensity greater than $13(0)$. 


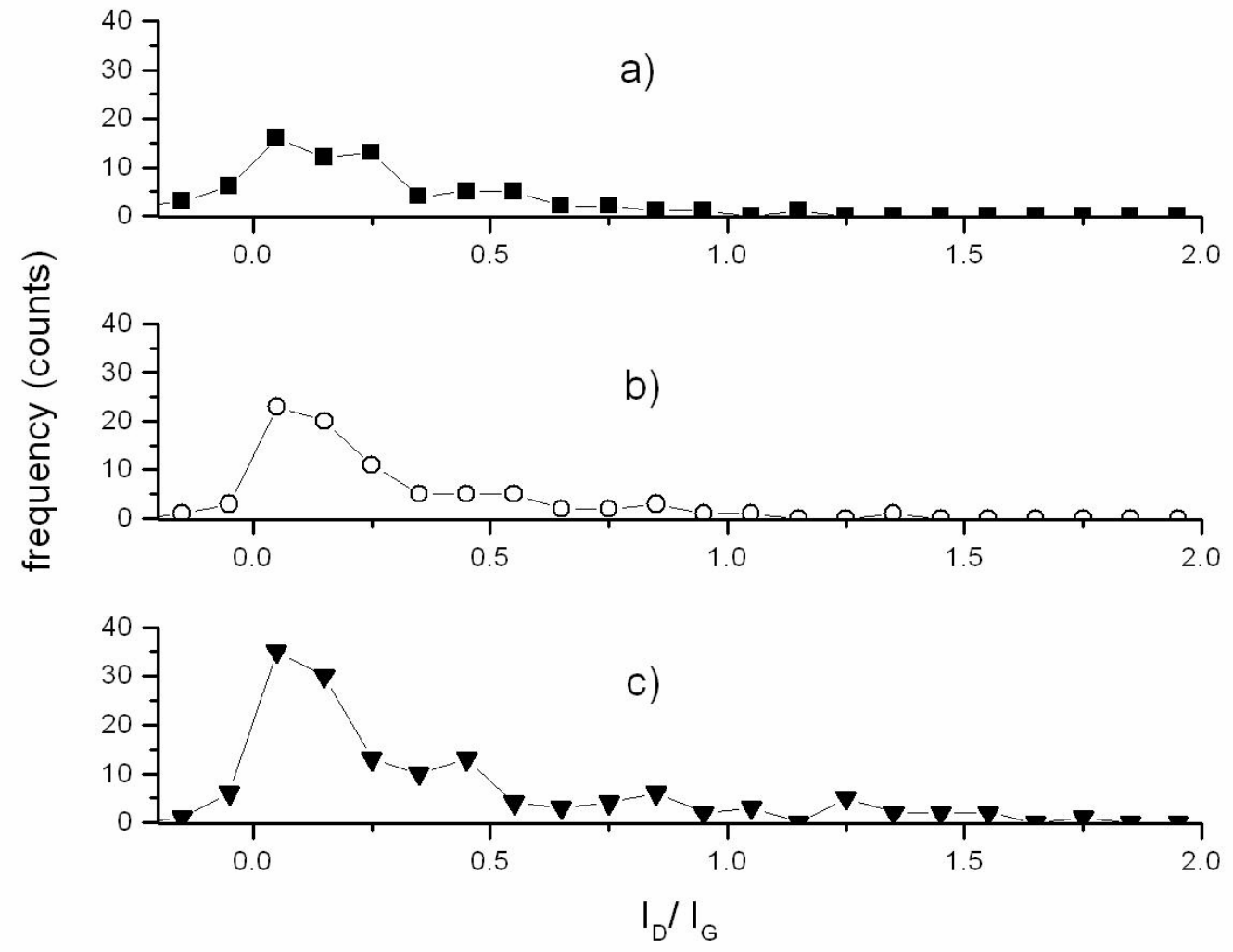

Figure 4.4. Histograms of the $\mathrm{I}_{\mathrm{D}} / \mathrm{I}_{\mathrm{G}}$ values of thresholded images without spectral preprocessing (a), with median filtration of the spectral data (b), and with IFT truncation of the spectral data (c). 

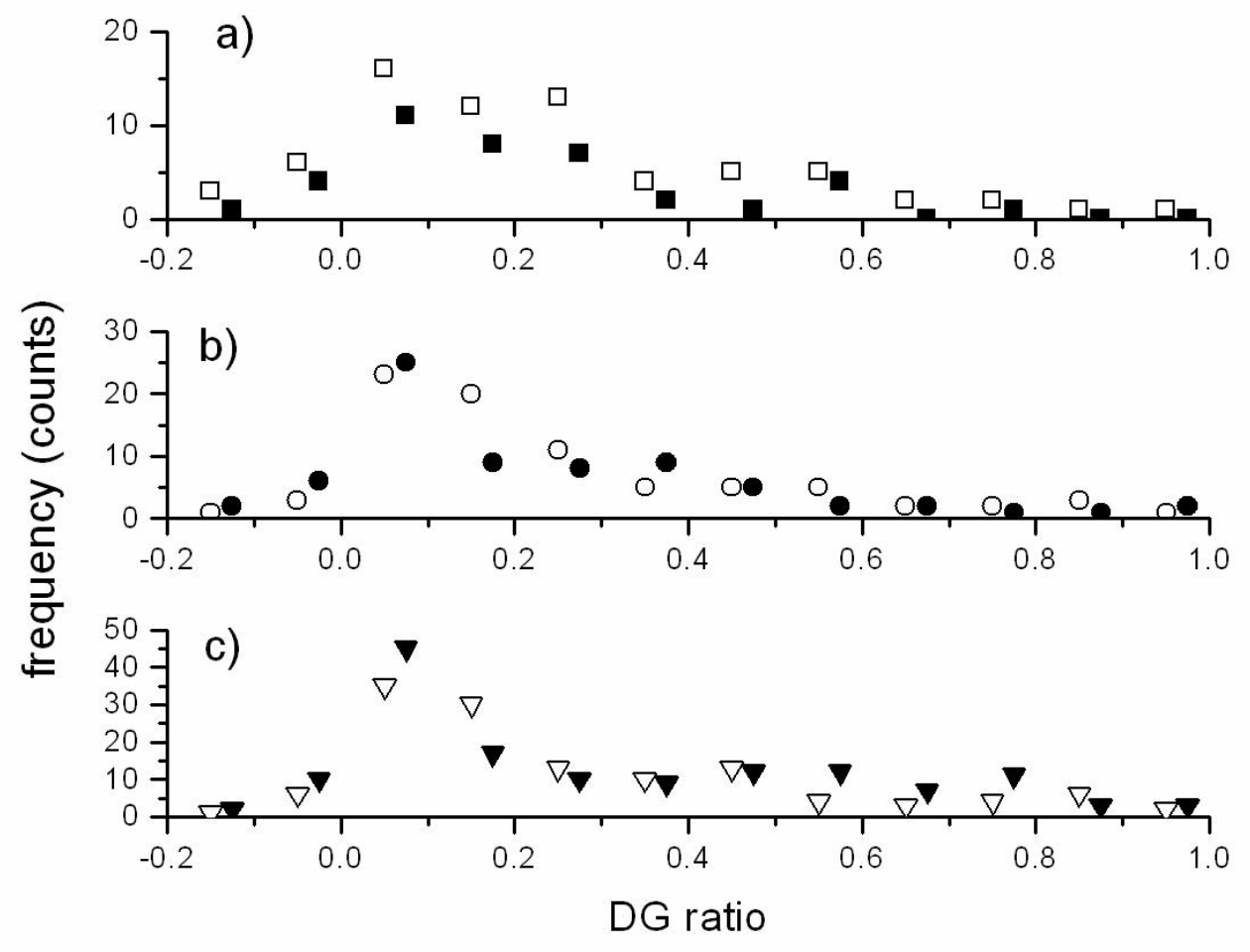

Figure 4.5. Histograms of the $\mathrm{I}_{\mathrm{D}} / \mathrm{I}_{\mathrm{G}}$ values for images acquired by point-mapping (solid) and line scanning (open): unprocessed spectral data (a), median-filtered spectral data (b), and IFT truncated spectral data (c). 

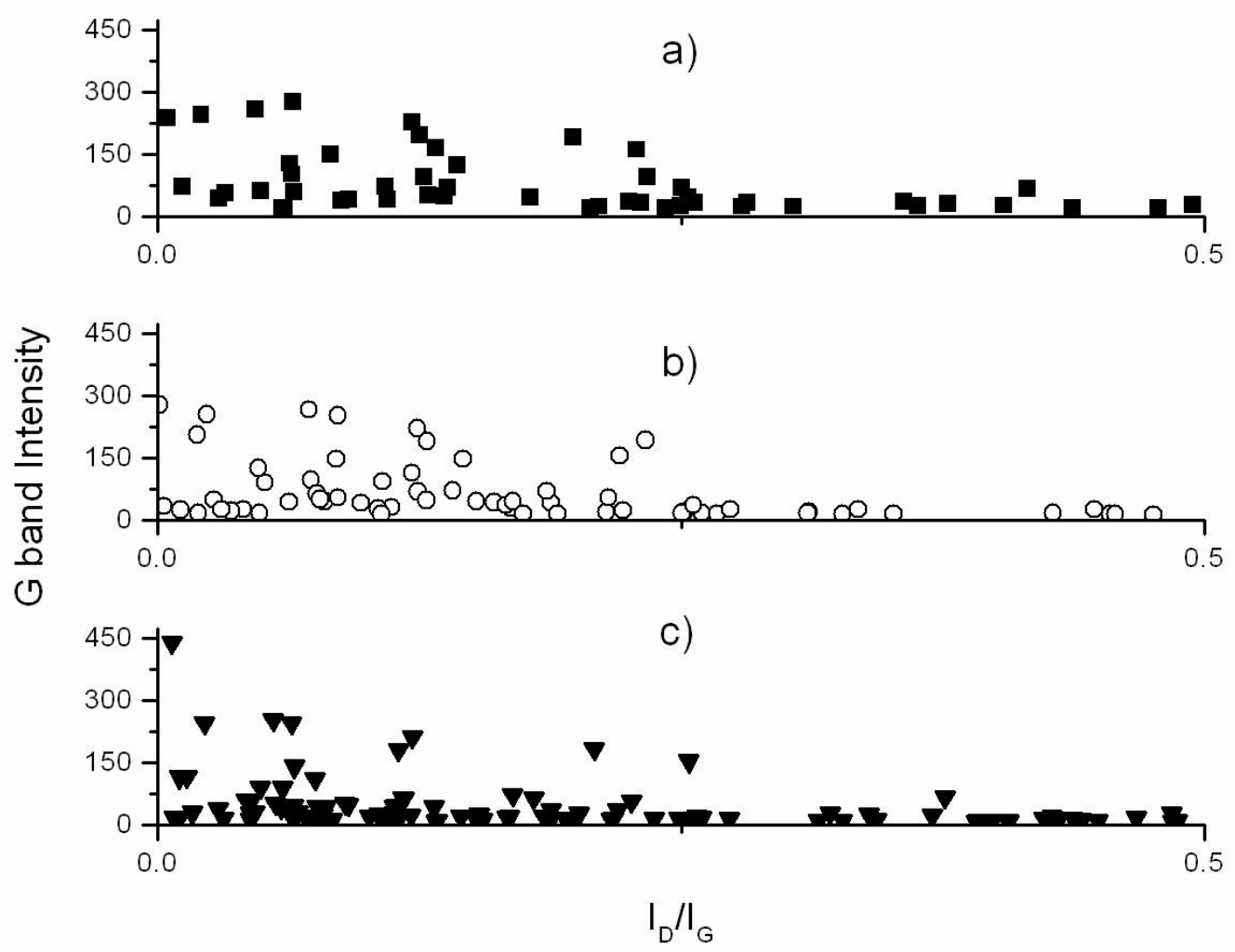

Figure 4.6. Scatter plots of $\mathrm{I}_{\mathrm{G}}$ versus the $\mathrm{I}_{\mathrm{D}} / \mathrm{I}_{\mathrm{G}}$ value for: pixels in the unprocessed image (a), the median filtered image (b), and the IFT truncated image (c). 